. 
GURO HERB 581.9
$(4)$ SWD 


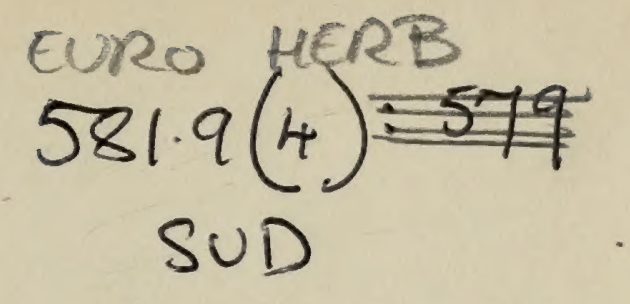









\section{H. SUDRE}

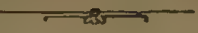

\section{BATOTHECA EUROPEA}

\section{Fasciculo I}

(1903)

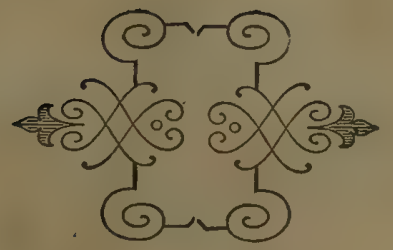

ALBI

IMPRIMERIE A. NOUGUIES

a

19083 


\section{BATOTHECA EUROPEA}

PUBLIÉ PAR

\section{SUDRE}

Professeur à l'Ecole normale d'Albi (France)

Parait par fascicules annuels de כ̋0 numéros.

Les collaborateurs qui fournissent 5 numéros préparés à 20-25 parts recoivent un fascicule en échange.

Chaque part doit comprendre, autant que possible, deux r'ameaux, l'un florifère, l'autre fructifère, plusieurs feuilles avec segment de tige de première année et quelques pétales préparés à part. Il est bon de noter, au sujet de chaque espèce, la couleur des pétales, des étamines et des styles.

\section{Prix du fascicule : $\mathbf{2 5} \mathbf{f r}$.}

\section{COLLEGTEURS POUR LE PREMIER FASCICULE}

Dr Bouly de Lesdain, à Dunkerque (France), nos 19, 20.

$\times$ T. Chaboisseau (France), nos 9, 17, 28.

D' w. O. Focke, à Brême (Allemagne), nos 7, 39, 44, 49.

$\times$ Gast. Genevier (France), nos 4, 12, 13, 26.

H. Hofmann à Grossenhain (Saxe), nos 2, 34, 35, 42 .

Jaquet, à Châtel (Suisse), nos 11, 41, 45.

F. Kretzer, professeur à Braunsweich (Allemagne), nos 3, 31, 37, 38.

$\times$ Levent (France) nos 30 .

$\times$ P.-J. Müller (Alsace), nos 29, 48, 50.

H. Sudre à Albi (France), nos 1, 5, 6, 9, 10, 14, 15, 16, 18, 21, 22, 23, $24,25,27,33,36,40,43,46,47$.

$\times$ Timbal-Lagrave (France), no 32 .

Ce fascicule renferme 22 Rubus qui n'ont jamais été distribués dans des exsiccata numérotés.

\section{OBSERVATION}

Après entente avec la Direction du musée de Lausanne, je dispose des centuries que le batologue alsacien P.-J. Müller avait réunies pour son Herbicr normal, qui ne parut jamais. Elles proviennent des récoltes de Chaboisseau, G. Genevier, Levent, Timbal-Lagrave et P.-J. Müller. Le $1^{\mathrm{e}}$ fascicule en renferme quelques-unes fort bien conservées; les autres seront distribuées ultérieurement.

H. SUdre. 


\title{
NOTES
}

\author{
SUR LES
}

\section{PLANTES DISTRIBUÉES}

$\supset$

Sect. I. - Suberecti P.-J. Müll.

- R. VITIDUS W. et N. Microg. R. oblongifrons Sud. - France: Tarn (H. sudre). - Plante voisine des $R$. hamulosus Lef. et M. et divaricatus P.-J. Müll. Diffère du R. hamutosus par ses folioles plue étroites, largement oblongues, ses rameaux peu armés et son inflorescence pauciflore portant de rares aiguillons falqués. S'éloigne du $R$ divaricatus par sa foliole caulinaire terminale à base plus élargie, émarginée, par ses aiguillons plus rares sur le rameau et sur l'inflorescence, qui est pauciflore, non divariquée; enfin par ses étamines plus longues et ses pétales rosés.

Est assez commune dans le Tarn et très constante dans ses caractères. Le turion est faiblement canaliculé ; la foliole caulinaire terminale assez courtement pétiotulée; les étamines sont blanches et dépassent peu les styles verdâtres; le calice est assez netternent réfléchi. La plante a le pollen très imparfait et ne fructifie souvent que partiellement. Toutefois, comme c'est la seule forme de cette section qui se rencontre aux environs d'Albi, il n'est pas possible d'y voir un produit de croisement.

2 - Th. SENTECOSO Kuhl. - Saxe (H. Hofmann). - La plante distribuée est plus généralement connue sous le nom de $R$. montamus Wirtg. (185\%). Mais comme il existe un $R$. montanus. Libert datant de 1813 et que, d'après les recherches faites par M. Du Pré (Cf. Compte-rendu séance 16 av. 1898, Soc. roy. bot. Belg. $t$. XXXVII '2 $p . p p$. 32-33), ce Rubus pourrait bien être l'espèce appelée $R$. leucandrus par M. Focke (1875), le nom de $R$. montanus ne peut plus servir à désigner l'espèce publiée par Wirtgen, qui paraît bien être la même que celle que Kœhler avait appelée R. senticosus (1829). 
Sect. II. - Silvatici P.-J: Müll.

Gr. a Grati Sud.

3- R. VURGMISW , et N. - Süd-Harz (F. Kretzer). - C'est le type de l'espèce, correspondant à la var. A viridis W. N. des Rub.germ.

4 - R. CLATHRophicus Gen. 1 Vendée (Genevier. - J'avais d'abord subordonné cette plante au R. vulgaris W. N., mais aujourd'hui que je l'ai vue d'une foule de localités en spécimens bien identiques entre eux, je suis porté à la considérer comme une espèce de $1^{\text {er }}$ ordre. Son inflorescence presque inerme et sa foliole caulinaire terminale la font reconnaitre aisément.

5- R. CLATHIROPHLCUS Gen. Subsp. F. Iarnensis Sud. - France: Tarn (H. Sudre). - Diftère du $R$. clathrophilus Gen. par sa foliole caulinaire terminale moins nettement obovée-cuspidée, sa denticulation plus grossière et ses organes floraux d'un beau rose vif.

AC. dans le Tarn et dans l'Aveyron où le R. clathrophilus est très rare.

$6-\times$ R. PSEUDO-TARNENSIS Sud. $(n . h y b)$. R. tarnensis $\times$ ulmifolius. - France: Tarn (H. Sudre). - Diffère du $R$. tarnensis Sud. par ses teuilles d'un vert sombre en dessus, nettement blanches-tomenteuses en dessous, à folioles plus larges et plus courtes, par ses sépales réfléchis et ses étamines courtes; du R. ulmifolius Schott par ses turions peu ou point glauques, ses feuilles pubescentes en dessous et son intlorescence presque inerme. Il est entièrement stérile.

Gr. b. Euvirescentes Gen.

7- T. MA A SoII Focke! - Hanovre (Dr W. o. Focke).

8 - M. QUESTMEIRI Lef. et M. S.-var. fatlax Chab. ! (pr.sp.). - Vienne) Chaboisseau). Chaboisseau dit ce Rubus commun et lui donne comme synonymes $R$. acuminalus Gien. et $R$. calvatus Bor. Il est manifeste qu'il visait le R. Qucstieri Lef. et M. dont Müller allait publier la description en mème tempsque celle du $R$. fallax. La plante qu'il avait 
envoyée à Müller, et que je distribue, n'est qu'une forme grêle, élancée, probablement jeune, du R. Questie$r i$. Le botaniste alsacien n'avait pas remarqué l'extrême affinité des deux formes puisqu'il les a publiées sous des $n^{\text {os }}$ très distants $(58,94)$ dans son Versuch. J'ai acquis la conviction qu'il connaissait mal son $R$. Questieri, pour lequel il avait exceptionnellement conservé le nom proposé par Lefèvre : dans la collection de de l'Isle j'ai rencontré de nombreux numéros de cette espèce auxquels il appliquait des dénominations nouvelles, ne paraissant pas reconnaître celle que son collaborateur avait dédiée à l'abbé Questier. Genevier ne faisait pas la distinction entre $R$. Questieri et $R$. fallax et peut-être y aurait-il lieu de l'imiter. Pour marquer la nuance qui sépare cette forme du type habituel, je l'ai envisagée comme une simple sous-variété.

9- T. QUEGTIERI Lef. et M. Microg. R. pyrenaicus Sud. - France : Cauterets (H. Sudre). J'ai vu de nombreux spécimens de $R$. Questieri et l'ai observé vivant dans six ou sept départements ; je l'ai toujours rencontré avec des fleurs roses et des styles carnés. Le $R$. pyrenaicus a constamment des fleurs à peu près blanches, même dans les lieux ombragés, et des styles verdâtres. Il est de plus beaucoup plus robuste que le $R$. Questieri, dont il me paraît constituer plus qu'une simple variété.

10 - TR. MI AROPHYLCUS W. N. Microg. R. calvifolius Sud. Rub. Pyr. p. 100 - France : Tarn (H. Sudre). - S'éloigne sensiblement du type macrophyllus par ses turions subarrondis et très légèrement pubescents ; par ses folioles presque glabres en dessous; par son inflorescence peu poilue, églanduleuse ; enfin par ses pétales très larges. La glabrescence de son feuillage le rapproche du $R$. Questieri Lef. et Mül. dont il diffère par ses fleurs blanches et ses turions pubescents.

Gr. c. Discoloroides Gen.

11 - R. MERTERT Gen. - Suisse (Jaquet.) - Il est possible que cette plante soit un hybride fixé dont la formule serait $: k$. candicans $\times$ vestitus. Mais dans ce cas le $R$. Mercieri devrait se rencontrer ailleurs, et jusqu'ici il n'est guère connu qu'en 
Suisse. Toutefois M. Bouvet a récolté en Maine-etLoire une forme peu éloignée de la plante de Mercier, et j'ai moi-mème rencontré dans le Tarn des formes que j'ai identifiées au $R$. Mercieri et qui ne sauraient dériver du $R$. vestitus puisque ce dernier manque dans les régions où j'ai récolté cette espèce. J'ai déjà fait observer (Rubus Herb. Boreau $p$. 53) que la plante de la Mayenne nommée $R$. Mercieri par Genevier était un hybride des $R$. adscitus Gen. et sprengelii Wh..(R. Chedceanus Nob.).

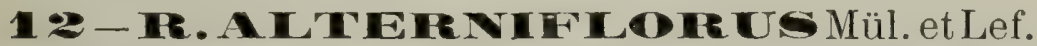
Sbsp. IR sepan'inug Gen. - Vendée (G. Genevier). - Le type $R$. alterniflorus M. et L. a les aiguillons bien égaux et est très peu glanduleux. Je crois que sa place exacte est avec les $R$. silvatici. Le $R$. separinus Gen., que j'ai pu observer sur place en 1901, me paraît plus rapproché du $R$. alterniflorus que de toute autre espèce; il est toutefois plus glanduleux et présente parfois un peu d'hété racanthie. Il est très abondant et très fertile et certainement pas hybride. Les fleurs sont parfois un peu rosées; le turion est assez nettement glauque sur le vif, caractère aujourd'hui peu apparent sur les spécimens absolument authentiques que je distribue.

$13-X$ R. MICROCARPUS Gen.! - R. separinus $\times$ ulmifolius sud. - Vendée (G. Genevier). Le No 483 de l'Assoc. rubol. distribué sous le nom de $R$. microcarpus ? et provenant de Beaucouzé (M. et L.) est un $R$. Genevier $i \times$ ulmifolius $=R$. breviatus Sud. et Bouv.

Sect. III. - Discolores P.-J. Mül.

Gr. a Gypsocaulon P.-J. Mül.

Ce groupe comprend tous les $R$. discolores à turion glauque : $R$. ulmifolius Schott, $R$. Godroni Lec. et Lm., $R$. Winteri P.-J. Mül. et $R$. propinquus P.-J. Mül.

14 - Re. ULMIFOLUUS Schott. Sbsp. T. vulgatus Sud. Rub. Pyr. p. 192. - France: Tarn $(\mathrm{H}$. Sudre). - Mercier appelait $R$. rusticanus les formes les plus diverses du groupe $R$. ulmifolius Schott et subordonnait à cette espèce, comme variétés, le $R$. bifrons Vest et quelques hybrides de $R$. ulmifolius et de 
tomentosus. On ne peut donc appliquer le nom de rusticanus à une formo particulière de ce groupe; c'est pour cela que j'ai appelé $R$. vulgatus la sousespèce définie par Genevier et par M. l'abbé Boulay (p. p.) sous le nom du $R$. rusticanus (sensu stricto).

15 - Microg. R. cruentiflorus Sud. Tarn (H. Sudre). - Le turion arrondi, la foliole caulinaire terminale orbiculaire, entière, cuspidée, les fleurs d'un rouge vif, font aisément reconnaître cette belle Ronce.

16 - R. GODMRONI Lec. et Lam. - Sbsp TR. Winteri P.-J. Mül. - Ariège (H. Sudre). - La plante que je distribue me paraît être assez exactement la même que celle que Winter avait récoltée aux environs de Sarrebruck et dont j'ai vu de bons spécimens dont la collection de $\mathrm{P}-\mathrm{J}$. Müller. Le $R$. Winteri n'est pas rare dans le centre de la France et je l'ai récolté dans la Corrèze, le Cantal et le Puyde-Dòme. L'Association rubologique l'a distribué de la Seine-Inf. (R. serratus Boul, et Let. ex parte), de la Saône-et-Loire ( $R$. cavaticaulis B. et Gill. ex parle). La collection des Rubi Gallici le renferme du Puy-deDôme, où je l'ai vu en abondance, sous le nom de $R$. longicuspidatus B. et Luc. var. discolor. Le R. Rivuli Boul. et Quincy, de Saône-et-Loire, en diffère à peine. Voilà donc une espèce jusqu'ici assez méconnue.

1 7 - IR. PHEOPINQUUSA P.-J. Müll.! Vienne.: Pindray (loco clas.) (T. Chaboisseau). Plante assez abondante sur tout le Plateau Central particulièrement dans le Cantal et dans le Puy-deDôme. On pourra peut-être un jour la subordonner au $R$. Godroni quand on aura trouvé des formes la reliant au $R$. IVinteri. Le $R$. immitis Bor. (non Genev.!) n’en est qu'une variété accidentelle, peu normale. Le $R$. dumosus Lef. Ass. rub. (ex parte: $420,485,542)$, dont le turion est assez nettement glauque, n'en est qu'une variété à inflorescence contractée. Müller, qui avait reçu cette plante de Lefèvre. l'avait du reste rapprochée de son propinquus. Le $R$. propinquus des Ronces vosgiennes $\mathrm{n}^{\circ} 106$ n'appartient pas à la même espèce.

M. N. Boulay ( $a p$. Rouy et Cam. $F l . F r . t$. VI $p . ; 9)$ considére la plante que je distribue comme un $R$. ulmifolius Xthyrsoideus. Comm cette Ronce est très 
fertile et qu'elle est assez commune dans tout l'ouest et tout le centre de la France, - je l'ai vue d'une douzaine de départements —, il est inutile d'insister pour montrer l'invraisemblance de cette hypothèse.

18 - X R. PILETODERMIS Sud. - $R$. propinquus $\times$ vestitus (Micr. leucanthemes). - Cantal : Aurillac (H. Sudre). - Diffère du R. propinquus P.-J. Mül. par son turion beaucoup plus velu; par ses feuilles coriaces, parsemées de poils en dessus, velues en dessous comme chez le $R$. vestitus W. N., à folioles plus courtes et plus larges, la terminale suborbiculaire, en cour, cuspidée; par son rameau très velu; par son inflorescence pius hérissée, très multiflore, à pédoncules étalés. Il s'éloigue du $R$. leucanthemus Müll. par son turion très glauque, anguleux; par ses aiguillons égaux et ses glandes nulles, par ses feuilles plus discolores et ses pétales moins larges, roses. Il est stérile et a un peu l'aspect d'un $R$. vestitus roséiflore. Le $R$. propinquus P.-J. Müll. est abondant aux environs d'Aurillac; il en est de même du $R$. leucanthemus dans les vallées de la Cère, de l'Allagnon, de la Jordanne, etc.

Gr. b. Hedycarpi Focke (pr. sp. coll.)

19 - IR. HEIFTMS Vest - France: Versailles (Dr B. de Lesdain).

$20-X$ R. PERROUDI Sud. $-R$. bifrons $\times$ ves titus; R. macroacanthos Perroud p. p. non W. N. - France : Versailles ( $\mathrm{D}^{\mathrm{r}} \mathrm{B}$. de Lesdain). - En société avec le $R$. bifrons Vest. Il en diffère par ses folioles beaucoup plus larges et plus poilues, qui lui donnent l'apparence d'un $R$. veslitus, par - ses rameaux et ses axes plus hérissés, par ses pétales plus larges et la présence de quelques rares glandes stipitées sur les pédicelles. Ses turions très anguleux, ses aiguillons égaux, très comprimés, ses glandes à peu près nulles, le distinguent du $R$. vestitus. Son pollen est entièrement atrophié.

21-TE.GTLITI N. Boul. var. nemophilus. Tarn (H. Sudre). - Ce Rubus, extrèmement commun dans le centre de la France et très constant dans ses caractères, ne paraît ètre une espèce de premier ordre 
ayant autant de valeur que le $R$. bifrons Vest. C'est certainement, après le $R$. ulmifolius, l'espèce la plus facile à reconnaître sur le vif.

$\boldsymbol{2} \mathbf{2}-\times$ R. PSEUDO-GILLOTI Sud. - R. Gillot $\mathrm{X}$ ulmifolius. - Tarn (H. Sudre). - Cet hybride, provenant du croisement de deux espèces voisines et d'une même section, est tout à fait stérile ; il en est de même de celui que j'ai appelé $R$. densiflorens (ulmifolius $\times$ Gilloti) Bul. Ass. pyr. n ${ }^{\circ}$ 283. Ces faits nous prouvent surabondamment qu'on ne saurait réunir en une même espèce collective tous les Rubus de cette section, malgré l'existence d'un certain nombre de formes très embarrassantes.

23 - R. PUIEEGCNS Wh. Microg. R. evagatus Sud. Bul. Ass. pyr. no 204. - France : Tarn (H. Sudre). - Très voisin du type $R$. pubescens Wh. En diffère par sa foliole caulinaire terminale courtement pétiolulée, son inflorescence moins lâche, munie de larges bractées, à pédoncules non ramifiés dès leur base, à pédicelles plus courts. Son pollen est de même très imparfait.

Le $R$. evagatus est très abondant dans le Tarn et dans le Cantal, à Aurillac, Ytrac, Yolet, etc.

24 - R. arpigens Sud. n. $s p$. - France: Tarn (H. Sudre). - Robuste ; turion élancé, faiblement canaliculé, glabre, à aiguillons vigourelux, très comprimés à la base. Pétioles à aiguillons denses, falqués ou crochus, très dilatés à la base. Feuilles grandes, glabres en dessus, grises ou blanches-tomenteuses et pubescentes en dessous, grossièrement et irrégulièrement dentées. Foliole terminale à pétiolule égalant la $1 / 2$ ou le $1 / 3$ de sa hauteur, largement ovale, échancrêe, brièvement acuminée; les inférieures pétiolulées. Rameau anguleux, glabrescent, à aiguillons forts, très dilatés à la base, fortement falqués ; feuilles la plupart 3-nées, grossièrement dentées, à foliole terminale ovale, échancrée, aiguë ou acuminée. Inflorescence grande, feuillée à la base, poilue, à aiguillons forts, nombreux, la plupart géniculés, queiques-uns crochus; pédoncules élatés à angle droil, très multiflores; pédicelles très aiguillonnés ; calice tomenteux, poilu, rarement aculéolé, rétléchi. Pétales ovales, retrécis en onglet, blancs uu faiblement rosulés ; étamines blan- 
ches, dépassant les styles verdàtres ; jeunes carpelles velus. Plante fertile, mais à pollen très imparfait.

Diffère du $R$. robustus Mül. (macrostemon Focke) par ses turions plus nettement canaliculés, glabres; ses feuilles plus grossièrement dentées ; son inflorescence plus lâche, bien plus armée, à pédoncules plıs étalés ; par ses pétales moins larges, et surtout par son pollen bien plus imparfait. Se rapproche beaucoup du $R$. agastachys Mül. et, Wirtg. Hb. rub. rh. Ed. I no 129 , qui a toutefois l'inflorescence beaucoup plus lâche, à pédicelles beaucoup plus longs, divariqués, à aiguillons moins denses et moins courbés, et le turion plus profondément canaliculé.

Plante commune dans l'Albigeois, ne paraissant pas d'origine hybride.

$$
\text { Gr. c- Thyrsoidei. }
$$

25-WE. TH YHSOIDIEUS Wimm. Subsp. TR. Iacertosus Sud. - France: Tarn (H. Sudre). - Se rapproche du $R$. robustus Mül. par ses pétales larges et son pollen peu mélangé. Il en diffère parses turions nettement canaliculés, glabres, et ses pédoncules moins étalés, plus ascendants.

\section{Sect. IV - Appendiculati Gen.}

Gr. a. Tomentosi.

26 - Te. TOMENTOSUS Borckh. Sbsp. F. CIoydianus G. Gen ! - Deux-Sèvres: (G. Genevier). - Bien que Genevier ait pu confondre sous ce nom des formes dérivées du $R$. tomentosus par croisement, je crois que la plante qu'il a particulièrement visée dans sa description et que je distribue, constitue plus qu'une simple variété du $R$. tomentosus (canescens). Elle est en effet très commune sur de grands espaces et douée par conséquent d'une certaine constance. Dans le Tarn le type canescens est à peu près introuvable tandis que le $R$. Lloydianus est très fréquent et ordinairement sans traces de tomentum à la face supérieure des feuilles.

27 - Tr. INCANNECiENS Bert. - 'Tarn (H. Sudre). - Cette plante, très abondante dans la Montagne-Noire (Tarn), appartient au même groupe 
que le R. tomentosus Borckh. Je possède des spécimens qui sont nettement intermédiaires entre les deux espèces et qui, ayant également le pollen parfait, ne sont certainement pas hybrides. Comme l'hétéracanthie est manifeste, au moins sur les turions, il m'a paru bon de détacher ce groupe des $R$. discolores de le mettre dans les Appendiculati Gen.

Gr. b. Vestiti Focke.

28 - T. CHE HODSGAEI P.-J. Mül. !Vienne (Chaboisseau). - On pourrait peut-être le subordonner au $R$. alterniflorus M. et Lef.; toutefois les turions sont plus arrondis, un peu glanduleux, à aiguillons plus fins, un peu inégaux, de sorte que sa place est plutòt dans les vestiti. D'un autre côté il se rapproche beancoup des $R$. macrostachys Mül., scabridus Mül., callichrous Sud., R. rubellus Miül., qui appartiennent plus manifestement à la section des $R$. appendiculati. Je le laisse provisoirement indépendant des formes voisines.

29 - R. VEGTITUG W. N. - Microg. R. leucanthemus P.-J. Mül.! - Alsace (Müller). - J’ai observé cette plante en grande abondance sur le Plateau central, en 1903, dans les vallées de la Jordanne, de la Cère, de l'Allagnon (Cantal), de la Dore, de la Dolore (Puy-de-Dóme) et ailleurs, et n'ai pu rencontrer, sur des terrains les plus divers, un seul buisson à fleurs roses. La coulem blanche des fleurs ne dépend ni de l'exposition, ni de la nature du terrain et n'est donc pas accidentelle: il y a par conséquent ici un caractère dont la fixité n'est pas douteuse, c'est pour cela que j'envisage cette forme comme une espèce de $3^{\mathrm{e}}$ ordre ou microgène.

30 - R. CONGPTCUUS P.-J. Mül. - sbsp. R. masmificus P.-J. Mül. - Marne (Levent). J'ai trouvé dans les Rubus légués au musée de Fribourg par feu Perroud, sous le nom de $R$. macroacanthos Cott. et Cast.! Guide bot. Frib. (ex parte) non W. N., un hybride de la formule $R$. bifrons $\times$ vestitus (R. Perroudi Sud. in hb. mus. Frib.) qui est tout à fait stérile et distinct du $R$. conspicurs. Il en est de même de la plante récoltée à Versailles par M. le $D^{r}$ de Lesdain (Bat. eur. n ${ }^{\circ}$ 20). Je crois que le $R$. conspicurs a la valeur d'une bonne espèce et je lui subor- 
donnele $R$. magnificus, plante que M. l'abbé Toussaint a récoltée dans l'Eure et qui s'observera sans doute aillèurs.

Gr. c. Radulce Focke.

31 - Re. RADUC. Wh. - Süd-Harz (F. Kretzer). - La plante de M. Kretzer paraît bien correspondre au type de l'espèce; c'est très exactement le $R$. decipiens P.-J. Mül. des environs de Wissembourg!

$\mathbf{3}$ - Microg. R. pustulatus Mül. inédit. - France : Environs de Toulonse (Timbal-Lagrave). - Diffère du type $R$. Radula Wh. tel que l'a récolté M. Kretzer par ses folioles caulinaires terminales profondément émarginées, par ses dents longuement mucronées et surtout par son inflorescence très vaste, ample, peu ou point feuillée, munie d'aiguillons droits ou déclinés, à pédoncules très allongés et très élalés à angle droit, portant des fleurs longuement pédicellées. Les fleurs sont petites, blanches. J'en ai vu de nombreux spécimens très homogènes provenant des localités suirantes : Hte-Garonne: Balma près de Toulouse, et forèt de Bouconne; Tarn-et-Garonne: bois de Grizolles. Il est commun dans ces stations, d'après Timbal, qui l'avait communiqué à Müller sous le nom de $R$. Brilleti; mais P.-J. Müller avait transmis au botaniste toulousain la note suivante : "C"est le $R$. pustulalus ! espèce commune répandue dans la plus grande partie de la France et qui tient de près au $R$. Radula W. N."

Je dois ajouter que je n'ai pas vu d'ailleurs cette curieuse plante et ne l'ai même pas encore trouvée dans le Tarn.

3:3 - Sbsp. 1t. orens Sud. (n. subsp.) - Cantal (H. Sudre). -- Turion glabre, anguleux, à faces planes ou un peu excavées, scabre, à aiguillons faibles, déclinés, inégaux, à glandes nombreuses, fines. Pétiole glabrescent, à aiguillons fins, déclinés. Feuilles la plupart 5-nées, glabres en dessus, grises-tomenteuses et brièvement pubescentes en dessous, à dents larges, inégales, superficielles ; foliole terminale à pétiolule égalant à peu près le 1'3 de sa hauteur, obovale, échancrée, brusqueinent acuminée. Rameau anguleux, pubescent, à aiguillons fins, déclinés ou falqués; feuilles :-nées, grises en dessous, à foliole terminale obovée. Inflorescence feuillée à la base, tomenteuse, 
courtement poilue, à glandes assez abondantes, mais courtes, à aiguillons pelits, droits ou déclinés ; pédoncules étalés-ascendants, fins, pluriflores, munis de larges bractées; calice tomenteux, poilu, peu ou point glanduleux, non aculéolé, réfléchi. Pétales d'un blane pur, ovales, rétrécis en onglet ; étamines blanches, dépassant les styles verdâtres ; jeunes carpelles glabres. Pollen assez imparfait.

A rapprocher du $R$. papulosus Mül. et Lef. dont il diffère par ses aiguillons beaucoup plus fins, ceux des pétioles non crochus, simplement déclinés, à base moins large ; par ses folioles plus élargies, à dents moins profondes; par son inflorescence moins lâche, à pédoncules plus ascendants, munis de bractées beaucoup plus larges; enfin par son pollen bien plus imparfait, contenant à peine $1 / 3$ de grains normaux au lieu de $5 / 6$

Plante commune dans le Cantal, particulièrement dans la vallée de la Jordanne où elle remonte jusqu'à Mandailles, et dans les bois du Lioran d'où elle descend jusqu'à Murat.

34-ME. FUSCUSWh. Microg. R. acanthodes Hofm.! (Saxe). - Par ses turions presque lisses et la pilosité de la face inférieure des feuilles, la plante se rapproche des $R$. vestiti. Toutefois l'hétéracanthie est très prononcée sur les rameaux, ce qui me la fait mettre avec les $R$. radula: Elle a le faciès du $R$. retrodentalus M. et Lef., mais en diffère par ses glandes beaucoup plus longues et ses feuilles plus velues en dessous.

35 - Fe. FOLIOSUS W. N. Sbsp. Te. pinicola Hofmann. - Saxe (H. Hofmann)! - La brièveté des étamines rapproche cette plante du $R$. dercisus M. et Lef; elle en diffère par ses folioles ovales, son inflorescence plus feuillée et plus hérissée et ses styles rouges.

36 - TR. INGETEICATUS P.-J. Mül. ap. Wirtg. - Microg. R. silvigenus Sud. - Puy-de-Dôme : Arlanc (H. Sudre). - Turion faible, obtusément anguleux, glaucescent, très poilu; aiguillons inégaux, faibles, un peu comprimés. déclinés ; glandes éparses, inégales ; pétiole à aiguillons fins, déclinés ou falqués. Feuilles la plupart 5-nées, à poils épars en dessus 
vertes et poilues en dessous, à dents fines, inégales. Foliole terminale à pétiolule égalant à peu près le $1 / 3$ de sa hauteur, étroitement ovale ou subrhombée, entière ou très faiblement émarginée, finement acuminée; les inférieures pétiolulées. Rameau un peu anguleux, très poilu, à aiguillons fins, déclinés, à glandes courtes, éparses ; feuilles la plupart 3-nées, rerles er dessous, à foliole terminale rhombée ou obovée. Inflorescence courte, peu feuillée, poilue, à aiguillons très faibles, à glandes éparses, plus courtes que le diamètre de l'axe; pédoncules étalés-ascendants, 2-3-flores ; pédicelles courts ; calice poilu, peu ou point glanduleux, non aculéolé, réftéchi; pétales oblongs, d'un beau rose; étamines rouges, dépassant les styles verdàtres ou plus ou moins rosés ; jeunes carpelles velus. Fertile.

Diffère du $R$. insericatus P.-J. Mül. par ses turions moins hérissés, ses feuilles moins poilues en dessous, présentant quelques poils épars, pectinés le long des nervures, plus finement dentées. Elle est commune dans la vallée de la Dore (Puy-de-Dóme) et vient aussi près de Bort (Corrèze) et à Labastide-St-Laurent (Ardèche).

3 - R. INEESTUS Wh. - Sudwest-Harz (F. Kretzer). - Cette curieuse ronce tient à la fois des $R$. vestiti et des radulce. Toutefois, sur la plupart des tiges qui composaient la centurie de M. Kretzer, l'hétéracanthie était manifeste et aussi prononcée que chez le $R$. Radula Wh. ; c'est ce qui me la fait placer dans le même groupe que cette dernière espèce.

Gr. d. Rudes Sud.

38 - R. TuDIS W. N. - Südwest-Harz (F. Kretzer). - Forme un peu grêle, mais à peu près typique.

39 - R. SCAmER Wh. - Allemagne $\left(D^{r}\right.$ W. O. Focke).

Gr. e. Hystrices Focke.

40 - It. KCEIILETE Wh. $s b s p$. R. doranus Sud. ; $R$. longicuspis Gen. (ex parte) non Mül. - Puyde-Dôme: Mont-Dore (H. Sudre). - Les échantillons 
que je distribue ont été cueillis dans un endroit ombragé et sont presque entièrement virescents. Ordinairement les feuilles sont nettement discolores en dessous. La plante abonde au Mont-Dore, particulièrement dans le vallon de la Vernière, et descend jusqu'à la Bourboule. Elle se reconnaît aisément à ses turions glabrescents, anguleux, armés comme chez le $R$. Koehleri ; à ses feuilles caulinaires la plupart 5-nées, très finement serrulées, ordinairement nette. ment discolores; à son inflorescence grande, très multiflore, feuillée à la base, nettement hérissée, à aiguillons abondants, firis, jaunâtres. Le calice est réfléchi ; la plante fructifie et il me paraît difficile d'y voir un vestitus $\times$ Bellardii. Le $R$. longicuspis P.-J. Mül. est une forme du groupe du $R$. Schleicheri Wh. et est bien distinct de la plante que je distribue. Ganevier avait groupé sous le nom incorrect de $R$. longiculpis des formes très hétérogènes.

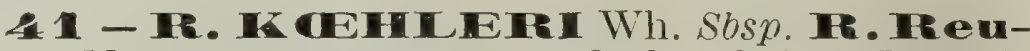
tori Merc. var. brevistamineus Sud. - Suisse (Jaquet). - Diffère du R. Reuteri Merc. par ses turions très peu glanduleux, ses folioles caulinaires terminales plus larges, à base un peu échancrée, les raméales moins nettement cunéiformes; par son inflorescence ordinairement nue et non très feuillée, à pédoncules étalés ; enfin par ses étamines un peu plus courtes que les styles. Le calice est vraisemblablement étalé, ce qu'on ne peut voir sur les spécimens distribués, qui ont été cueillis trop tôt.

Gr. f. Glandulosi P.-J. Mül.

42 - M. SCHLIECHETI Wh. - Saxe $(H$. Hofmann). - Je considère la plante distribuée comme représentant bien le type de l'espèce, qui a été en partie mal figurée par Weihe et Nees. Le $R$. spinulosus P. J. Mül. in Flora p. 169 est à peu près la même plante.

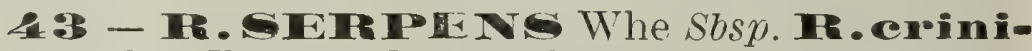
tus sud. - France: Tarn (H. Sudre)._Reconnaissable à son turion très glauque, très poilu; à ses feuilles très mollement poilues, veloutées en dessous, tendant à devenir discolores; à son inflorescence arquée, très hérissée, et à ses étamines très courtes. Ses carpelles sont glabres; fructifie abondamment. 
42 - TR, HELLATEII Wh. - Allemagne (Dr W. O. Focke).

25- R. VIIIARGSINUS Focke. Suisse (Jaquet). - Si cette plante provenait du croisement des $R$. hirtus et cosius on devrait la rencontrer ailleurs qu'en Suisse, les parents présumés n'étant pas rares dans la plupart des régions montagneuses. Il est vraisemblable que c'est plutôt une forme de transition entre ces leux espèces. L'hybride des $R$. hirtus et cosius, que j’ai signalé dans les Pyrénées, est tout à fait stérile tandis que le $R$. Villarsianus fructifie abondamment.

Sect. V -- Triviales P.-J. Mül.

16 - $\times$ R. AMPLIFOI folius $\times$ coesius (hyb. coll.). var. a vulgaris. France: Tarn (H. Sudre). - Je distribuerai prochainement les var. thanmocharis (Mül.) et acontophorus (Mül.).

$27-\dot{X}$ R. OREIFORMIS Sud.; $R$. oreus $\times$ cosius - Cantal (H. Sudre). - Diffère du R. oreus par ses turions moins glanduleux et moins scabres, un peu glaucescents; par ses stipules larges; par ses folioles plus amples, souvent 3-4 nées, plus profondément dentées, la terminale largement ovale-suborbiculaire, cordée, aiguë ; par ses rameaux peu glanduleux; par son inflorescence plus courte, à glandes peu visibles, et par ses pétales orbiculaires. Ses turions anguleux, à aiguillons un peu comprimés; ses feuilles grises-tomenteuses en dessous; son inflorescence allongée, multiflore, munie de larges bractées, et ses sépales réfléchis le distingurent du $R$. ccesius L. Il est stérile.

Il n'est pas rare dans le Cantal, particulièrement dans la vallée de la Jordanne. Les spécimens que je distribue croissaient pêle-mèle avec le $R$. oreus, à l'entréc de la vallée d'Albepierre, près de Murat.

18 - IR. CAESIUS L. - R. ligerinus Gen. - Alsace (Müller). - Cest sous la forme ligerinus Gen. que le $R$. ccesius se rencontre à peu près partout : il y a done lieu d'envisager cette forme comme le type de l'espèce. La forme que Genevier considérait 
comme typique n'est qu'une variation de son R. rivalis, d'après les spécimens authentiques que j'ai vus dans l'herbier de Müller.

$$
\text { Subg. Idæobatus Focke. }
$$

$\mathbf{A} \mathbf{9}-\times$ R. IDEOIDES Ruthev. pseudo-casius(Lej.) - Allemagne ( $\mathrm{D}^{\mathrm{r}}$ Focke). - Obtenu artificiellement par notre savant collaborateur M. le Dr Focke en fécondant le $R$. Idceus par le $R$. coesius. Le $R$. cosioIdacus P.-J. Mül., dont je possède une bonne centurie récoltée par Müller lui-même, est à peu près la même plante.

50 - TR. ID AEUS L. V. inermis G. Br. Alsace (Müller).

\section{H. SUDRE.}

Novembre 1903.

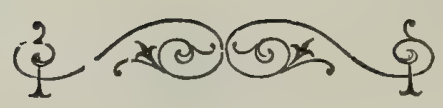





\section{DU MEAME AUTEUR :}

Les Hieracium du Centre de la France, d'apris les types de Jordan et de Boreat, avec 32 pl, contenant 200 fig.

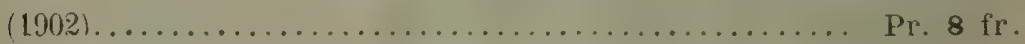

Les Rubus de l'herbier Boreau, étude sur les espèces de G. Genevier (1902)........................

Rubus des Pyrénées françaises (Description, analyse, tables),

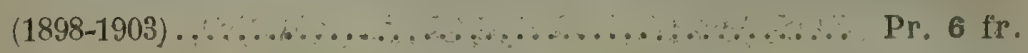

Révision des Rubus de l'herbier du Tarn, de De MartrinDonos.................................. Pr. $1 \mathrm{fr}$. 
Batotheea

$$
\text { Fax II }
$$





\section{BATOTHECA EUROPAA}

PUBLIÉ PAR

\section{H. SUDRE}

Professeur à l'Ecole normale de Toulouse (France)

Allées St-Michel, 19

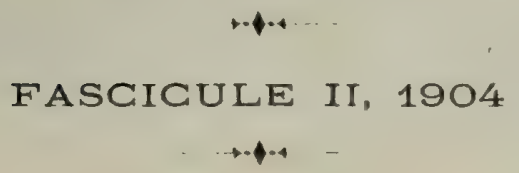

Parait par fascicules annuels de 50 numéros.

Les collaborateurs qui fournissent $\ddot{j}$ numéros convenus d'avance et préparés à $20 ̈$ bonnes parts, reçoivent un fascicule en échange.

\section{Prix du fascicule : 25 ir.}

\section{COLLECTEURS POUR JE 2 FASCICULE}

$\times$ T. Chaboisseau (France), no 78 .

D' W. O. Focke, à Brême, (Allemagne), no 96 .

$\times$ G. Genevier (France), $n^{\circ} 62$.

A. Gentil, professeur au Mans, Sarthe (France), nos 73, 79.

F. Gravet, à Louette-St-Pier're (Belgique), $n^{0}$ s $51,55,56,87,90,94$.

A. Henry, au Maus, Sarthe (France), no 79.

H. Hofmann, professeur à Grossenliain (Saxe), nos 58, 60, 95.

Jaquet, à Châtel (Suisse), nos $69,8 \%$.

Jourdes, agent principal d'assurances, à Verneuil, Eure (France), nos 52,61 .

F. Kretzer, professeur à Braunsweich (Allemagne), $10 \div 76,97,100$.

$\times$ Levent, Marne (France), no 81 .

Rv. E. F. Linton, Salisbury (Angleterre), nos 77, 80.

$\times$ P.-J. Müller (Alsace), nos 53, 92, 99.

D' H. Sabransky, ì Snchau (Autriche), nos 74, 75, 86.

G. Sampaio. jardin botanique de Porto (Portugal), $11^{0 .} 66,88$.

H. Sudre, professeur à 'Toulouse (France), $11^{05} 54,57,64,65,67,68$, $71,82,83,85,89,91,93,98$.

$\times$ Timbal-Lagrave (France), nos $63,70,72$.

Ce fascicule renferme 26 Rubus qui n’ont jamais été distribués dans das exsiccata numérotés.

J'ai recu des collaborateurs beaucoup d'autres Rubus qui n'ont pu trouver place dans le 20 fascicule, mais qui seront distribués dès que l'étude en sera faite.

H. SUDRE. 
Sect. I. - Suberecti P.-J. Müll.

$\mathrm{N}^{0} 51$ - Me. SUMETRCTU Ands. Belgique : Louette-St-Pierre ( $\mathrm{F}$. Gravet).

La description du $R$. Nessensis W. Hall. s'appliquant aussi bien au $R$. fissus Lindl. qu'au $R$. suberectus Ands., je crois, avec M. Focke, qu'il est préférable de faire usage du terme d'Anderson pour désigner la plante représentée par le $n^{0} 51$. J'ai vu des spécimens d'Anderson identiques à ceux que distribue M. (đravet. La planche 2 des Rubi germanici représente sous le nom de $R$. fitstigiatus (ex p.) un rameau de $R$. suberectus (M. Focke).

\section{No 52 - TE. NITHDUG IV. N. Sbsp. Fe. ho-} len'yhrus Focke. - Eure : forêt de Breteuil (Jourdes).

Le nom le plus ancien donné ì cette forme serait celıi de R. pleonacanthus appliqué par Müller en 1861 (Bonplandia) ì de beaux exemplaires trouvés à Beaumont près de Valogne (Manche) par Lebel. La plante est longuement décrite dans les mémoires laissés par Müller mais n’a pas été publiée.

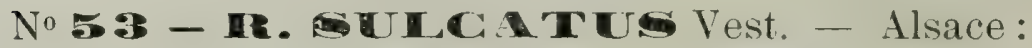
Wissembourg (P. J. Müller).

Le no 9537 de l'Herbarium europreum de M. Baenitz, cueilli en Silésie, est un $R$. sulcatus très normal que $\mathrm{M}$. le Dr Utsch considère comme un $R$. macrophyllus $\times$ candicans. En France le $R$. sulcalus n'est pas rare dans le midi où le $R$. macrophyllus manque; cette observation, quel'on pourrait appuyer de beaucoup d'autres considérations, montre l'invraisemblance de l'origine hybride du $R$. sulcatus Vest.

La table II des Rubi germanici (R. fastigiatus IV. N.) figure des feuilles caulinaires de $R$. sulcutus Vest et un rameau de $R$. suberectus And. D'après M. Focke, à qui est due l'observation précédente, pareille confusion existait dans les exsiccata de Weihe. Le nom de $R$. fastigiatus est donc à rejeter. Je $R$. sulcalus a parfois des turions à faces planes, même sur des pieds vigoureux. 
Sect. II. - Silvatici P.-J. Müll.

Gr. a. Grati Sud.

No 5 - Re. nepentinus Sud. - Tarn (H. Sudre).

Turion anguleux, à faces planes ou un peu concaves, glabrescent, non glandulcux, à aiguillons égaux, comprimés. Feuilles 5 -nées, à quelques poils en dessus, la plupart grises-tomenteuses et pubescentes en dessous, finement dentées; stipules à quelques glandes courtes; pétiole plan, à aiguillons déclinés ou falqués; foliole terminale suborbiculaire, en coev, brusquement acuminée, à pétiolule égalant environ la $1: 2$ de sa hculeur, les autres larges, se recouvrant par les bords. Rameau obtusément anguleux, pubescent, à aiguillons déclinés ou falqués, à feuilles grises-tomenteuses en dessous. Inflorescence courte, liche, pauciftore, souvent corymbiforme, lrès trièvement poilte, à quelques glandes courtes, peu apparentes, à aiguillons ordinairement assez robustes, droits, déclinés on un peu falqués ; calice gris-tomenteux, ordinairement aculéolé et parfois un peu glanduleux, à lobes un peu appendiculés, élalés ou lichement apprimés; pétales étroitement ovales, blanos ou un peu rosulés ; étamines blanches ou à base un peu rosée, dépassant à peine les styles verdâtres ou à base rosée; jeunes carpelles glabrescents ou glabres. Plante très fertile.

On peut provisoirement la rapprocher du $R$. vulgaris W. N. dont elle diffère par sa foliole caul. terminale suborbiculaire et longuement pétiolulée, caractère qu'elle a de commun avec le R.rhamnifolius W. N. Je la posède de 3 localités du Tarn; elle a été récoltée à Nantes et à Auverné (Loire-Inférieure) par de l'Isle.

Il n'en existe qu'un buisson dans la forêt de Sérénac, de sorte que les parts distribuées sont un peu maigres, mais elles montrent suffisamment les caractères de l'espèce.

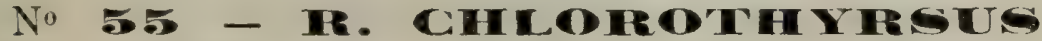

Fock. var. Micradenes. - Belgique: Prov. de Namur (F. Gravet).

Diffère du type de l'Allemagne septentrionale par ses turions plus anguleux, glaucescents, glabrescents ; par ses folioles caulinaires terminales largement ovales, nettement cordiformes, très acuminées; par ses glandes très peu visibles sur les axes et les pédi- 
celles, presque sessiles ; entin par ses pétales plus étroits.

No 56 - He. OFTHOCLADUS A Ley. Belgique, prov. de Namur ( $\mathbf{H}$. Gravet).

Le R. orlhocladus N. Boul. Ronc. eosy. 127 (1869) étant la même plante que le $R$. anoplostachys P.-J. Mül. (1861) d'après M. N. Boulay lui-même (ap. Rouy et C. $F l$. $f r . t$. VI $p$. 122), le núm donné par M. le Rév. A. Ley peut être conservé à cette remarquable espèce. Le $R$.orthocladus A. Ley est bien distinct des $R$. Sprengelii Wh., Arrhenii Lge, hemislemon P.-J. Mül. par ses turions glabrescents ou glabres, son inflorescence brièvement et peu poilue, à glandes abondantes, à pédoncules ascendants; ses sépales verdàtres, bordés de blanc, apprimés sur le fruit, et ses étamines longues. Il est plus manifestement hétéracanthe que les autres Rubus de ce groupe et se rapproche un peu de quelques formes de la série des $R$. rudes Sud. M. M. Rogers (Handb. of Brit. Rub.) le place dansles R. restiti.

\section{Gr. b. Euvirescentes Gen.}

No - Ariège : Aulus (H. Sudre).

J'ai d'abord présenté cette plante comme une sousespèce du $R$. Questicri Lef. et M. ; mais ce dernier a le turion glabre tandis le $R$. elongalispinus l'a courert d'une pubescence courte mais dense, ce qui me porte à l'envisager comme une espèce de $1^{\text {er }}$ ordre, au mème titre que le $R$. fagicola de Martr., que M. N. Boulay subordonne également au $R$. Questier $i$, mais à tort selon moi. Le $R$. elongatispinus parait spécial aux Pynénées, où il est assez commun.

No 58 - T. SICASACUSWh. - Allemagne : Dresde (H. Hofmann).

No $59-$ Te. GICVA'THCUG IV. N. - Allemagne : Brême (I) Focke).

Gr. c. Discoloroides Gen.

No 60 - re. VILtICAULIs Kohl. Saxe (H. Hofmann).

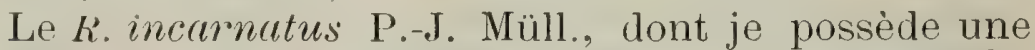
centurie récoltée par Müller lui-même, est sensible- 
ment distinct du R. villicaulis Koehl. Je le publierai prochainement.

No 61 - IR. A RGENTEUSW. N. Subsp. R. consobrinus Sud. var. RosefrLorus. - Eure : Acou (Jourdes).

s'éloigne du type du midi de la France par ses aiguillons plus forts, surtout dans l'inflorescence, ses folioles moins larges, ses tleur's à organes colorés ; en est sans donte une forme aprique. Le turion glabrescent, la foliole caul terminale émarginée, les bractées églanduleuses. le séparent du R. argenteus Wr. N. dont il est voisin, d'après notre savant collaborateur M. le Dr Focke. Séloigne beancoup du type revillicuulis Koehl, auprès duquel je l'ai placé dans mes rubus des Pyrénées p. 46. Vient aussi dans le Finistère, le Morbihan, la loore-Inféricure (de l'Isle), l'Anjou (Bouvet), le Poitou (de Lackoix), le T'arn et le Porthgal (G. Sampaio!.

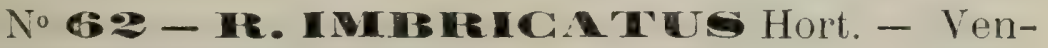
dée : Mortagne (Genevier).

J'ai observé sur quelques rares spécimens de cette flante que le calice restait étalé ; c'est évidemment une exception. Des individus semés en 1901 et ayant flenri en 1904 pour la $1^{\text {re }}$ fois ont le calice réfléchi ; il l'est aussi manifestement dans la centurie de Genevier. Il vaut donc mieux placer cette espèce dans les $R$. discoloroides, au voisinage du $R$. rhamnifolius $\mathrm{W}$. N. Par quelques-unes de sés variétés (var. Cariensis en particulier) elle se rapproche beaucoup du $R$. affinis $\mathrm{IV}$. N. On ne saurait toutefois la mettre dans les $R$. suberecli; encore moins dans les $R$. triviales comme le faisait (ienevier, qui dit les pétales " orbiculaires à onglet court " alor's qu'ils sont ovales et rétrécis en onglet a la base. Varie à feuilles presque toutes rertes en dessous ou la plupart discolores. Le type d'Angleterre est plus franchement virescent : "Leatlets...plaler and pilose benaeth" (Bab.l.c.) Encore une espèce passablement polymorphe.

Sect. III. - Discolores P.-J. Müll.

(†r. a. Gypsocaulon P.-J. Müll.

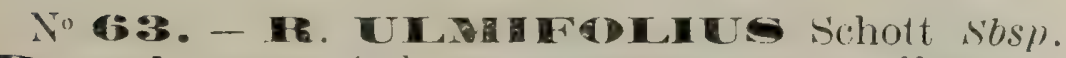

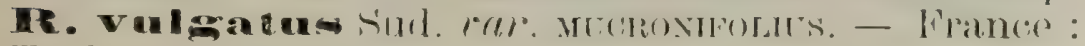
Toulouse (Timbal). 
Variété raconnaissable à ses folioles caulinaires terminales courtement obovées, tronquées et assez longuement apiculées au sommet; à dents inégales, acérées ; intlorescence inerme ; fleurs roses. Serait commune à Toulouse, d'après l'étiquette de Timbal.

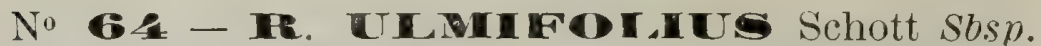
Fe. anisodon Sud. var. Genuinus. - Tarn (H. Sudre).

Cette sous-espèce comprend toutes les formes à foliole caulinaire terminale largement elliptique ou plus souvent obovale, ordinairement entière à la base, toujours atténuée-aiguë ou acuminée au sommet, jamais tronquée-cuspidée. La denticulation est irrégulière et les aiguillons pétiolaires sont crochus.

No 65. - IR. TIMTE DLUE Schott $S b s p$. Fe. dilatatifoliens Sud. - Tarn : Albi (H. Sudre).

Foliole caulinaire suborbiculaire ou largement ovale, ordinairement entière, aiguë ou cuspidée; denticulation assez irrégulière. Ləз folioles caractéristiques sont celles des feuilles prises vers le milieu du turion; celles de la base sont parfois émarginées, et celles du sommet ont une tendance à devenir obovées. C'est autant que possible dans la région moyenne que les feuilles caulinaires de $R u b u s$ doivent ètre prélevées.

No 66 - Re. PROPINRUUS P.-J. Mül. Sbsp. Te. Caldasianus G. Samp.! - Portugal : montagnes de Gérez (G. Sampaio).

Diffère du $R$. propinquus P.-.J. Mül. par ses folioles plus obovales, manifestement poilues en dessus, ses fleurs blanches et ses aigruillons moins comprimés. Ses turions et ses rameaux sont aussi moins anguleux, plus arrondis, caractère qui rapprocherait la plante du R. Godroni Lec. et Laint. Toutefois, par le tomentum et la pilosité de la face inférieure des feuilles. par la denticulation asse\% irrégulière et la villosité des axes florifères, ce Rubus me parait plus rapproché du $R$. propinquus que du R. Godroni. - "La plante est une des espèces les plus balles du Portugal, fréquente dans les montagnes du Noid, et bien distincte par ses belles fleurs blanches, semblables à celles du poirier. ") (G. Sampaio, in lit.). 


\section{HYBRIDES}

No 6 - $-X$ R. MODESTIFRONS Sud. - R. BIFRONS $X$ ulmifolius. - Tarn : Brassac (H. Sudre).

Rappelle le $R$. bifrons Vest par la forme de ses folioles, leur denticulation fine et son turion non glauque. Il en diffère toutefois par son inflorescence plus làche, à axe peu poilu, ses tleurs d'un rose plus vif, tout à fait stériles, à pollen entièrement atrophié. S'es feuilles ont le tomentum ras du $R$. ulmifolius schott et les folioles raméales sont obovées-cuspidées comme dans la sous-espèce $R$. vulgalus sud. de ce dernier. J'ai récolté ce mème hybride dans la vallée du Dadou, au pont de Grandval, et à la côte du Pont de Cirou (Tarn).

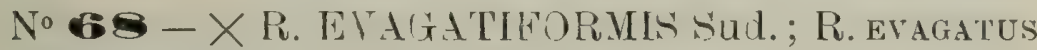
$X$ ulmifolius. - Tarn (H. Sudre).

Principaux caractères du R. evagatus (Batot. No gs). En diffère par ses feuilles d'un vert plus foncé en dessus, à tomentum presque ras en dessons, à foliole terminale moins acuminée; par son inflorescence bien plus vaste, multiflore, moins poilue, ses pétales plus larges, suborbiculaires, roses, ses étamines moins longues. Ces différences sont dues à l'influence du R. ulmifolius sichott qui a joué ici le ròle de portepollen. Plante à peu près stérile.

\section{Gr. b. Hedycarpi Focke.}

No 69 - He. 1691BU TUS P.-J. Müll. Suisse: Canton de Fribourg (F. Jaquet).

I'après M. l'abbé Boulay (Rév. aut. des Rub. discol.) Müller aurait " singulièreinent varié dans l'interprétation de son $R$. robustus ». Or, en faisant l'étude de la collection du batologue alsacien j'ai acquis la preuve que Müller groupait sous ce nom des éléments très lomogènes, identiques au type distribué dans les Ronces rosgiennes $n^{\circ} 7$. Les échantillons de Steinselt\%, du 30 juin 1858 , que M. Boulay alpelle R. macroslemon $\mathrm{v}$. robustus, ne diffèrent pas de la forme des Vosges. Mïller, qui ne procédait quarec une extrême jrudence à l'ilentification de denx formes de Rulues, est très aftimatif all sujet do nombrenx spésinens de R. robuskes provenant de coblenee de lonse. de la

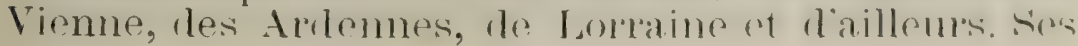


étiquettes portent des observations telles que les suivantes : "Type de mon $R$. robustus ; c’est là le véritable $R$. robustus ; ceei me parait un $R$. robustus - des plus normaux!; $R$. robustus, ce qu'il y a de plus authentique !!! etc. " Or les plantes ainsi appelées correspondent au $R$. macrostemon (pr. max. p.) de la Flore de France, qui est aussi celui de M. Focke. Le nom de Müller doit donc être conservé.

\section{G. c. Candicantes Focke.}

J'avais appelé ce groupe $R$. thyrsoidei dans le $\mathbb{1}^{\text {er }}$ fasc. du Balotheca ; mais ce terme a été employé par Halacsy et Braun pour désigner un ensemble très complexe de Rubus et appliqué par Genevier à un mélange de formes hybrides et d'espèces appartenant en partie au gr. hedycarri Focke. Jadopte le terme de M. Focke tout en plaçant dans le groupe des $R$. subtomenlosi le R. Arduennensis Lib.

\section{No 70 - HE. THYISOMDEUS Wimm.} Sbsp. Re. candicans Wh. var. COARCTATUS P.-J. Müll !·(pro. sp.) Alsace (P.-J. Müller).

Turion canaliculé ; foliole caulinaire terminale ovale, échancrée, brièvement acuminée; folioles raméales, simplement aiguës ou peu acuminces, la terminale ovale ou elliptique, à contour assez net par suite de la denticulation peu irrégulière des feuilles. Inflorescence plus ou moins lâche, peu ou point feuillée, à pédoncules rscendanls, peu armés ; pétales ovales, pâles. Les nombreuses variétés que présente le $R$. candicans étant fréquemment confondues, j'ai donné les caractères de la var. courc/atus d'après les spécimens que je distribue et que Müller considérait comme typiques, sauf quelques lameaux appauvris provenant d'un fourré, entre La Chapelle et St-Germain, qui sont accompagnés des étiquettes de Müller luimême!

No $11-X$ R. POIIANTHUS P.-J. Müll. ; R. CANdicans XLloydianus Sud. - Tarn: Terssac (H. Sudre).

L'influence du R. Lloydianus Gen. est manifeste; celle du $R$. condicans Wh. se déduit du turion canaliculé, glabre ou ì peu près, et de la forme des folioles, qui sont assez étroites. Le R. polyanthus P.-J. Müll. 
du Tersuch, $\mathrm{n}^{\circ} 30$, me parait ètre un $R$. collicolus $\times$ Lloydianus (=R. candens Sud. Rub. Herb. de Martr.)

\section{Gr. d. Subtomentosi Sud.}

Plantes intermédiaires entre les 3 groupes précédents et $R$. tomentosus Borckh., toujours fertiles et ne paraissant pas hybrides. Peut-être hybrides fixés?

\section{(Timbal). \\ $\mathrm{N}^{0}$ / $\approx$ - Re. collicolus sud. - Toulouse}

Les parts que je distribue sont un peu maigres, surtout en feuilles caulinaires. Cela tient à ce que la 12 des feuilles de la centurie de 'T'imbal étaient étrangères à cette espèce et appartenaient au $R$. ulmifolius. Le $R$. collicolus a le pollen ordinairement peu mélangé et fructitie bien. Il est possible que ce soit un hybride fixé dérivé du $R$. tomentosus Borckh. ; toutefois il est assez commun en france sous plusieurs formes et se rencontre souvent là oì le $R$. tomentosus manque. I a plante n'est nullement hétéracanthe et je ne vois pas qu'on puisse la subordonner au $R$. tomentosus ainsi que je l'avais d'abord fait. Bien que queloues parts n’aient que des feuilles caulinaires 3-nées, celles-ci sont le plus généralement 5̆-nées.

\section{Sect. 1V. - Appendiculati Gen.}

Gr. b. Vestili Focke.

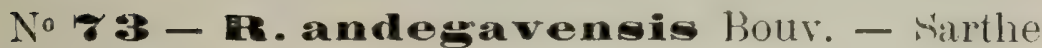
(A. Gentil).

Cette curieuse plante a le pollen assez imparfait et ne fructifie parfois que partiellement. Elle est asse\% commune en Maine-et-Loire (M. Bouvet) et se rencontre dans la Sarthe (M. Gentil) et dans la Loire-Inférienre (de Lisle). Son origine hybride me paraît des plus douteuses. Elle se rapproche du R. restilus IV. N. par ses axes florifëres très hérissés et du $R$. conspicuus P.-J. Müll. par ses feuilles glabrescentes en dessus et peu poilues en dessous. Son tırion glauque, ses folioles non orbiculaires, sa denticulation très superficielle, ses glandes rares et peu apparentes la distinguent aisément de ces deux espèces. 
No 7 \& - 1R. POHOPITYLUS P.-.J. Müll. Microg. R. holochlorus Sabrs. - Autriche : Styrie orientale (Dr A. Sabransky).

Plante très voisine du type $R$. podopluyllus P.-J. Müll. de Gérardmer (Vosges). En diffère par ses folioles caulinaires terminales plus larges, suborbiculaires, cordiformes et longuement pétiolulées, par ses glandes plus courtes dans l'inllorescence et ses étamines moins longues. M. le Dr Focke la considère comme une variété de son $K$. epipsilos (Syn. p. 258) dont elle paraît différer surtout par sa denticulation simple et superficielle et ses féuilles vertes et légèrement pubescentes en dessous, à folioles plus larges.

No 75 - Te. basalticaneum Sud. ver. Avaricus Sabrs. - Autriche : Styrie orientale (Dr H. Sabransky).

Ne differe de la plante d'Auvergne que par ses aiguillons caulinaires un peu plus forts et ses folioles caulinaires terminales nettement émarginées à la base. Paraît aussi moins bien fructifier. M. Sabransky me l'a alressée sous le nom de $R$. feslicus M. et Wirtg. var. cuaricus; mais le $R$. festivus est beaucoup plus glanduleux et plus hétéracauthe et me parait appartenir au groupe des $R$. hystrices $\mathbf{F}$., ce qui n'est pas le cas de la plante distribuée.

No 76 - It. SRITMTIDER Y NUS Sud. var. Longiglandulosus. -- Allemagne : süd-Harz ( $\mathrm{F}$. Kretzer).

Le $R$. Schmidelyanus comprend tous les Rubi vestili à turion anguleux, poilu, à feuilles caul.. 5-nées, poilues en dessous, à inflorescence hérissée et à calice étalé.

Le No 76 diffère dı type de Friboury par ses turions moins velus, ses feuilles plus pubescentes en dessous, à poils plus manifestement pectinés le long des nervures, ses dents plus superficielles, son inflorescence plus fortement poilue-hérissée et munie de glandes très longues. Il rappelle la var. urvernensis sud. par la forme et les caractères de son inflorescence, mais en diffère par sa denticulation plus régulière et ses feuilles bien plus poilues en dessous.

Le $R$. terctiuscules Kalt., Wirtg. Hb. rub.rh. Ed. I, 101 (p. max. p.) et ed. II 4:3 (pr. max. p.) a le turion nettement arrondi, des aiguillons courts, l'inflorescence moins hérissée et munie de glandes courtes. 
Il me paraît spécifiquement distinct du $R$. Schmidelyanus, bien qu'appartenant à la même série.

Gr. c. Radula Focke.

\section{No 7 - TR. HADULA Wh. sbsp. IR. eni-} cotorum Lef. - Angleterre: Bournemouth (E. F. Linton).

J'ai recu la même plante de la sarthe et la publierai prochainement. Je la considère comme distincte du $R$. uncinalus P.-J. Müll. des Vosges. Le R. fissipctalus Gen. (non Müll.) n'en diffère pas sensiblement. J'ai vu plusieurs spécimens nommés $R$. citralus par Ġenevier qui n'étaient pas distincts du $R$. ericetorum Lef.

\section{No 88 - R. GRVIER Bor.! - Vienne:}

Pindray (T. Chaboisseau).

Cette plante est très constante dans ses caractères et asse\% commune dans l'Ouest de la F'rance. Notre distingué collaborateur, M. Gonçalo Sampaio, l'a décourerte en Portugal et m’en a adressé de beaux spécimens. Je suis porté à la considérer comme une espèce de $1^{\text {or }}$ ordre.

No 79 - He. obovatifrons sud. - Sarthe (A. Gentil et A. Henry).

Turion anguleux, à faces planes ou un peu concares, glabre ou à poils rares, non glauque, à glandes inégales, très hélércuccultie, à grands aiguillons comprimés, plus ou moins obliques ou même falqués. Stipules étroites, glanduleuses; pétiole à aiguillons courts, falqués on croctus. Feuilles j̀-nées, ì quelques poils épars en dessus, rertes ou les supérieures grises et pubescentes en dessous, lies finement et superficiellement dentées, à dents aiguës, la plupart simples; foliole terminale à pétiolule ne dépassant guère le 1 i) de sa hanteur, comrtement obovale on obovaleorbiculaire, un peu émarginée à la base, brusquement arcuminér oul copirlée; les infépieures brièvenent pétiolulées. Rameau anguleux, glubrescent, glanduleux, à aiguillons très inégaux, cour $/ s$, les plus forts falqu's, quelques-uns gémiculés ou crochus ; feuilles :3-nées, finoment dentés, is foliole terminale obovale, large, cuspidée, les sunérioures parfois un pen discolores. Intlorescence comple. Iargr, an pen interrommure of fenillée à la base, trompuce an sommet, lichement 
poilue, glanduleuse, ì aiguillons pâles, nombreux, déclinés on falqués; pédoncules moyens étalés-ascendants, multitlores; sépales tomenteux, très hérissés, un peu glanduleux et aculéolés, non ou peu appendiculés, réfléchis; pétales blancs, ovales; étamines blanches, dépassant les styles verdâtres; carpelles glabres. Pollen mélangé ( $1 / 2$ de grains normaux).

Voisin du R.granulatus Mül. et Lef. En diftère par ses turions glabres, plus scabres, ses folioles plus élargies, ses rameaux glabrescents, son inflorescence plus courte et plus large, à villosité plus làche, et ses pétales moins étroits; il est aussi plus hétéracanthe et a une tendance à devenir discolore, ce qui le rapproche du $R$. Radula Wh.

No 80 - Re. RANUATOS. et L. Microg. R. melanodermis Focke. - Angleterre: Bournemouth. (E. F. Linton).

Le type $R$. granulalus M. et L. a été cueilli dans l'Oise par Lefèvre et dans les Ardennes par Callay! Ce n'est certainement pas un $R$. sprengelii X macrophyllus, ainsi que l'admet M. N. Boulay (ap. R. et C. Fl. Fr. VI p. 106). Le R. melanodermis Focke n'en diffère guère que par son inflorescence plus dense et moins glanduleuse ! Il n’en est peut-être qu'une variété.

$\mathrm{N}^{\circ} 81$ - R. FOLIOSO Wh. et N. Microg. R. flexuosus Müll. et Lef.! - France : Marne (Levent).

La détermination de cette plante est due à Müller lui-même! D'après Levent elle est très commune dans les bois de la Marne et très polymorphe. J'ai vu dans l'herbier Müller des spécimens de Gremli appelés $R$. saltum par M. Focke qui m’ont paru identiques â la plante que je distribue. Le $R$. flexuosus M. et L., qui n'est peut-être qu'une variété du $R$. foliosus W. N., en diffère par ses folioles caulinaires terminales elliptiques, ses dents plus superficielles et plus fines, ses pédoncules très peu poilus, courts, souvent ramifiés dès la base et à pédicelles fasciculés; ses feuilles sont ordinairement coriaces et un peu discolores.

Gr.d. Rudes Sud.

No 8 2 - TE. MELA MOXYLN Mül. et W. var. GLABRESCENS - Tarn (H. Sudre).

Diffère du type par ses feuilles glabrescentes en 
dessous, son intlorescence moins feuillée et plus dense; ses styles roses. Le calice est étalé.

Il existe dans le Morbihan, à Vannes (Arrondeau), des buissons qui correspondent exactement à la plante de Wirtgen. Le R. perdurus Hol. et Borb. (R. durus Hol. prius non Sauvage), d'après les spécimens de Holuby que j'ai en herbier, se rattache au $k$. melanoxylon M. et W.

No 83 - R. supenbus Sud. - Tarn (H. Sudre).

J'ai décrit la plante comme ayant un turion non glauque; mais depuis j'ai remarqué qu'on observe assez souvent un peu de glaucescence sur les tiges de première année, et c'est le cas dans les spécimens distribués. La plante est intermédiaire entre $R$. Guenther $i$ W. N. et $R$. ulmifolius Schott, mais est toujours très fertile et n'en est probahlement pas hybride. Elle se place entre les R. rudis W. et N. et scaber W. N. tout en étant bien distincte de ces deux espèces.

Gr. e. Hystrices Focke.

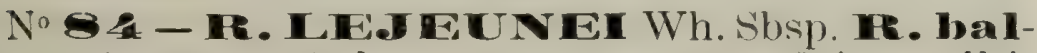
moariensis sud. var. Reversus. - Suisse : Hribourg (Jaquet).

Mes spécimens de $R$. balneariensis de Luchon ont le calice imparfaitement rétléchi. D'autres échantillons cueillis par M. Schmidely dans le canton de Fribourg ont de même le calice étalé, de sorte que le $R$. balneariensis tel que je l'ai décrit paraît bien exister en Suisse. Toutefois dans les spéciınens récoltés par M. Jaquet et que je distribue, les sépales se réfléchissent nettement à la tin, tout en restant assez longtemps étalés. A noter aussi que l'inflorescence est plus dense et a les pédoncules plus ascendants. Par la forme de l'inflorescence la plante se rapproche du $R$. Lejeunei Wh., mais par ses turions glabres et ses folioles larges elle tend vers le R. rosaceus W. N.

No 85 - R. erythranthemus. - 'Tarn : Sérénac (H. Sudre).

Gréle et élégante. 'Turion subarrondi, rougerilie', glancescent, glabrescent, tres glanduleux, à aiguilIons très inégaux, les grands comprimés, déclinés ou droits. Feuilles ln pluparl 3-nées, quelques-unes 1-5nées, à poils apprimés en dessus, les moyenmes et 
les supérieures grises ou blanches-lomenteuses et à nombreux poils brillants en dessous, très finement et presque simplement denticulées; foliole terminale courlement obovale, échancrée, aiguë ou un peu acuminée, à péliolule égalant environ les 2.5 de sa hauteur. Rameau pen anguleux, flexueux, peu poilu, ḋ nombreuses glandes pourpres, à grands aiguillons déclinés, rarement falqués; feuilles supérieures discolores, à foliole terminale large, obovée, cuspidée. Inflorescence courte, large et liche, peu ou point feuillée, courlement poilue, à nombreuses glandes pourpres, très inégales, à aiguillons médiocres, droits ou déclinés; pédoncules moyens étalés, dirisés en pédicelles longs et divariqués; calice tomenteux, courtement poilu, glanduleux, quelquefois un peu aculéolé, imparfaitement réftéchi; pétales grands, ovales, diun rouge vif ainsi que les étamines et les styles ; étamines très longues; jeunes carpelles un peu velus. Pollen peu mélangé, pur aux 9/10 environ.

Les feuilles discolores et finement dentées le distinguent du R. Lojeunoi Wh. Est à rapprocher des $R$. mutabilis Gen., rubrans Müll., angusticuspis Sud. (R. setrulosus Rogrers non M. et I.), morvennicus Gill. et d'un certain nombre d'autres formes affines que l'on peut subordonner au $R$. oblruncatus P.-J. Mül. Le R. formidabilis Baenitz Herb. eur. 10365 (non Lef. et M.) paraît très voisin du R. erythranthemus.

No 86 - Tt. pilocanpus (Gremli. - Autriche: Styrie (Dr H. Sabransky).

Tout à fait identique aux spécimens de (tremli provenant des environs de Zurich ! Se place dans le voisinage des $R$. mutabilis (ten., erinaceus schmid.. expolitus sud., etc., que l'on peut rapprocher du $R$. obtruncatus P.-J. Müll. (1858). ('e dernier ne diffère guère du R. pilocarpus que par ses turions glabrescents, ses folioles moins larges et sa denticulation moins pro. noncée.

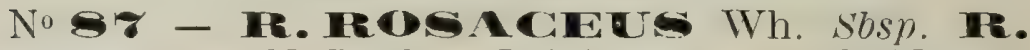
conomatus N. Boul. - Belgique, prov. de Namur (F. Gravet).

La plante de Belgique, bien qu'à styles colorés et à sépales presque réfléchis, paraît bien être la même que celle de Phalempin (Nord). L'hypothèse de M. l'abbé N. Boulay, qui considère ce Rubus comme un R. Spren- 
gelii $\times$ foliosus, ne me paraît pas admissible : la glaurescence du turion, la denticulation grossière des feuilles, l'aculéation si remarquable de l'inflorescence, la longueur des étamines, sont des caractères qui ne peuvent provenir d'aucun des parents présumés. J'estime, avec M. (travet, le collecteur de ce No., que le $R$. coronalus doit être envisagé comme une forme pure.

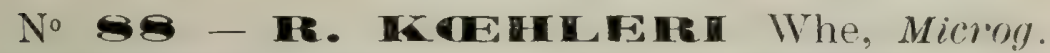
R. gereziacus (*. Sampaio! - Portugal: Mont. de Gere\% (G. Sampaio).

Diffère du type par ses aiguillons moins denses, ses feuilles souvent : '-nées, glabrescentes en dessous, à denticulation beaucoup plus vive, sa foliole caulinaire terminale plus rétrécie et presque entière à la base, insensiblement et longuement acuminée, son inflorescence peu ou point feuillée, à axe beaucoup plus poilu; enfin par ses sépales non réfléchis et son pollen encore plus imparfait. Fructifie partiellement.

Le $R$. Kichleri Wh. manque probablement dans le sud-ouest de l'Europe et il me paraît difticile de ne voir dans ce $n^{\circ} 88$ qu'une simple variété de cette espèce.

Gr. f. Glandulosi P.-J. Müll.

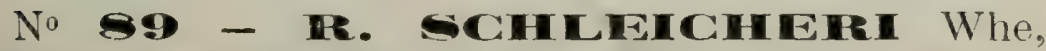
Microg. R. fulvus sud. - Tarn (H. Sudre).

Forme caractérisée par ses turions glabres, pou ou point glauques; ses aiguillons forts, denses, la plupart fortement géniculés ou vivement crochus ; par ses folioles étroites, rhombées, son inflorencence glabrescente, très armée, à glandes longues et à aiguillons forts et denses ; enfin par ses étamines longues et ses calices réfléchis.

No 90 - TR. MEIUC IEIS Mïl. et Wirtg. Microg. R. leptobelus Sud. - Belgique: Pros. de Namur (F. Gravet).

Turion glabre, non glauque, fortement glandılouxaciculé. Feuilles :-j-nées, vortes et pubescentes on dessous, grossièrement dentées foliole caul. terminale courlement pétioludré, oblongne-rhombre, insensible-

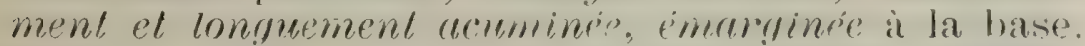
Rameau glubrescent, it feuilles grossierement dentees. 
Inflorescence lichc, plus ou moins feuillée, pâle, à axe non poilu. Pédoncules et sépales portant de nombreuses soies jaunes; calice fructifère étalé ; étamines égalant les styles pâles. Carpelles velus.

Se rapproche dı $R$. horridulus P.-.J. Mül. par ses turions glabres, mais en differe pas ses feuilles grossièrement dentées, à folioles de forme très différente. Il ressemble un peu au $R$. viridis Kalt., mais ce dernier a le turion et le rameau visiblement velus, l'axe de l'inflorescence un peu poilu et les étamines longues.

Le $R$. leptobelus a été récolté dans la Vienne, au Rond, allée de Dauzé, par l'abbé de Lacroix (Herb. Müller).

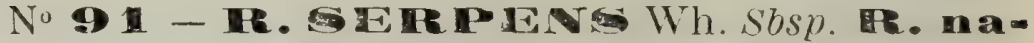
pophiloide sud. - Puy-de-Dòme (H. Sudre).

La plante distribuée a été récoltée dans un endroit très frais et le turion est bien moins poilu que d'habi tude, presque glabrescent. Je crois, malgré cette légère différence, que ce Rubus appartient à la même forme qu'un grand nombre d'autres que j'ai groupés sous ce nom dans mon herbier. Le $R$. napophilus Mül., des environs de Wissembourg, en diffère par sa denticulation plus vive, a dents longuement mucronées et plus ou moins divariquées, ses folioles longuement acuminées, son inflorescence courte mais très lâche, à pédoncules longs et très étalés à angle droit, à pédicelles presque divariqués; ses étamines dépassent à peine les styles. Il appartient aussi au groupe du $R$. serpens Vhe tel que je le comprends.

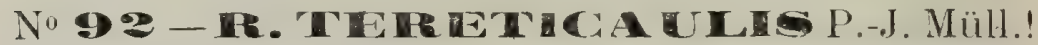
- Alsace (P. J. Müller).

Le $R$. tereticaulis P.-J. Müll. est assez souvent mal interprété. Il est surtout caractérisé par son inflorescence très brièvement poilue, munie de glandes courles, la plupart de longueur inférieure au diamètre des pédoncules. J'en possède de nombreuses formes qui seront publiées ultérieurement.

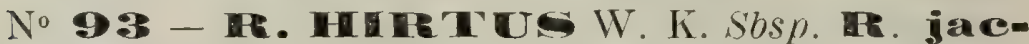
tabundus sud. .. France: Cauterets (H. Sudre).

La plante a été cueillie dans un lieu très ombragé et est moins rougeâtre que les formes habituelles du R. hirtus. Flle est remarquable par sa denticulation très fine, superficielle, ses folioles étroites et son inllorescence poilue-hérissée. 


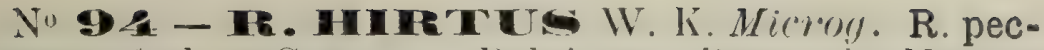
tinatus sud. et Grav. - Belgique: Prov. de Namur (H. Gravet).

I'urion subarrondi, glaucescent, très poilu, ì glandes nombreuses, à acicules rares, à aiguillons faibles, les grands un pen comprimés ì la base. Feuilles :-nées, it quelques poils épars en dessus, vertes et très jubescentes en dessous, a poils abondants et pectinés le long des nervures comme che\% le R. myramidalis Kalt., à dents très superticielles, médiocres, inégales, la plupart simples ; foliole terminale ovale-rhomboïdale, un peu émarginée et faiblement acuminée. Rameau trés poilu, à aiguillons médiocres, déclinés ; foliole raméale terminale obovale-cunéiforme, entière, superficiellement dentée. Inflorescence petite, courte, feuillée, très poilue-hérissée, à aignullons médiocres, à glandes abondantes, ronges, la plupart dépassant un jeu le diamètre des pédicelles; calice poilu, glanduleux, aculéolé, à lobes étroits, étalés ; pétales étroits, blancs ; étamines blanches, bien plus courtes que les styles roses; jeunes carpelles glabres.

II. le $\mathrm{D}^{\mathrm{r}}$ Focke (in herb. F. Fravet) a rattaché cette forme comme var. brevistamineus au $R$. mbiginosus P.-J. Mül. Le type R. rubiginosus du Pigeonnier, plès de Wissembourg, a le turion glabre, les feuilles glabres en dessous, l'inflorescence à axe glabrescent, les étamines longues et les pétales larges; il me parait totalement distinct de la plante de Belgique.

\section{Sect. V. - Triviales P. J. Müll.}

N" 95 - $\times$ R. DISSIMULANS Lindg.; R. PLicates $X$ czsius Sud. - Saxe (H. Hofmann).

L'intervention d'une forme de la sect. des $R$. subcrecti dans la production de ce numéro est assez manifeste. La brièveté des étamines ne peut être due qu'au R. plicalus $\mathbf{W}$. N. Bien que les aiguillons caulinaires soient faibles, j'en ai l'emarqué quelques-uns de nettement comprimés à la base; de plus le $R$. Holandrei P.-.I. Mül., que je considère comme un R. suberectus $X$ cresius, à la denticulation beaucoup plus fine.

No $96-X$ R. LUMECTORUM šnd.; R. HOLERYTHRES $X$ cesius. - Allemagne ( $D^{r}$ Focke).

Cette forme correspond en partie au $R$. corylifolius des batologues anglais et II. Hocke me l'a adressée 
sous le nom de $R$. corylifolius var. flor. roseis. Je crois que la pubescence de la face inférieure des feuilles, les nombreux aiguillons de l'inflorescence, la coloration rose des pétales et des étamines, ne peuvent provenir que du $R$. holerylhrus Focke, qui n'est pas rare dans le sud de l'Angleterre.

Ne diffère pas sensiblement de la plante que j'ai récoltée entre Mortagne et Evrunes (rendée) en 1901.

No $\mathbf{9} \mathbf{\gamma}-\times$ R. SUlCa TIFORMis Sud. $-\mathrm{R}$. SUlcaTus $X$ cæsius. - Allemagne : süd-Harz (F. Kretzer).

Sous ce nom je comprends tous les hybrides plus rapprochés du $R$. sulcalus Vest que du $R$. casius L. et paraissant provenir de la fécondation du premier par le second. Dans les beaux exemplaires cueillis par M. Kretzer la prédominance du $R$. sulcatus est manifeste. J'ai vu de nombreux spécimens de cette forme dans l'herbier P.-J. Müller et provenant des environs de Wissembourg; l'un était rattaché à tort au R. virgultorum P.-J. Mül. et les autres ne portaient aucun nom. Gremli appelait $R$. fruticosus le $R$. sulcalus Vest. Ce qu'il a décrit sous le nom de $R$. coesius-fruticosus, Beilr. p. 24 paraît bien être la inême plante que le R. sulcaliformis. Il existe d'autres hybrides plus rapprochés du $R$. coesius que du $R$. sulcatus qui correspondent à la formule $R$. coesius $\times$ sulcatus.

No $98-X$ R. ASSURGENS Boul. et Bouv. var. vulgaris; R. coesius $\times$ ulmifolius - Tarn : Cambon $(\mathrm{H}$. Sudre).

Bien que plus rapproché du $R$. cresius que du $R . u l$ mifolius Schott, ce Rubus porte l'empreinte du R. ulmifolius (vulgalus) dans la forme de ses folioles caulinaires terminales, qui sont un peu obovales.

Le nom de $R$. patens Merc. est bien antérieur à celui de $R$. assurgens; toutefois Mercier appelait $\boldsymbol{R}$. patens non seulement toutes les formes issues du croisement des $\boldsymbol{R}$. ulmifolius et cosius, mais encore des hybrides de la forme tomentosus $\times$ casius et autres, ainsi que je l'ai constaté dans l'herbier P.-J. Müller! Ce nom, manquant de précision, est donc à rejeter.

$\mathrm{N}^{\circ}$ 99- $\mathrm{P}$ R. CUSPIDATUS P.- J. Müll. ; $R . R a-$ dula $\times$ coesius Sud. - Alsace (P.-J. Müller).

Les rameaux de cette plante ressemblent beaucoup 
¿ ceux du R. Raduta et Mïller, en 185\%, étiquetait ce Rubus : R. Radula Wh. Le R. cuspidalus des Ronces vosyiennes, $\mathrm{n}^{0} 19$, a l'inflorescence moins poilue, presque inerme, munie de glandes plus courtes, la denticulation plus fine. Je le considère comme un $R$. obsectifolius $\times$ coesius $=R$. cuspidatiformis M. in $h b$. Müller. Dans la centurie de Müller j’ai trouvé un assey. grand nombre de rameaux qui appartenaient visiblement à une autre forme de la inème section ; toutefois la description du Flor a, faite à l'aide des matériaux récoltés en $185 \%$, vise uniquement la forme que je publie.

No 100- C. C.AOUS L. Microg. R. spiculatus Boul. et Bouv. - Allemagne: süd-Har\% (F. hletzer).

M. F. Kretzer m'a adressé cette forme sous le nom de $R$. cossius X rudis fa supercesius. Il n'est pas impossible que ce soit un hybride en voie de retour an $R$. c $(\boldsymbol{\ell}-$ sius L.; toutefois l'influence de l'autre parent est si peu marquée qu'il me paraît bien dificile de préciser lorigine de la plante. Je crois plutòt qu'elle dérive du $R$. cesius L. par simple variation; son pollen est en effet pur ou très peu mélangé, mais il est, lans beaucoup de fleurs, détruit par un champignon microscopique qui provoque une stérilité partielle en mème temps que le développement exagéré des sépales et de quelques pétales (macropétalisme). Je ne rois pas de différence appréciable entre ce $\mathrm{n}^{\circ}$ et le $R$. spiculatus Boul. et Bouv. tel que M. Bouvet lui-mème me l'a fait récolter à Angers, étang St-Nicolas, et qui me paraît se rattacher au $R$. ccesius comme forme pure. Le $R$. rivalis Gen. en est très voisin mais il a les pódoncules et les sépales munis de glandes courtes et rares.

\section{H. SUDRE.}

Novembre 1904. 



Exour $15, x+1=$

$\because$ is int

$$
\therefore+-
$$

$-$

$$
\therefore \quad \therefore 0+\frac{111}{4}
$$





\title{
BATOTHECA EUROPEA
}

PUBLIE PAR

\author{
II. SUDRE \\ Professeur à l'ḱcole normale de Toulouse (France) \\ Allées Saint-Michel, 19

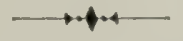

FASGICULE III - 1905

Parait par fascicules annuels de 50 numéros.

Les collaborateurs qui fournissent 5 numéros convenus d'avance et préflarés à 200 parts, reçoivent un fascicule en échange.

\section{Prix du fascicule : 25 fr.}

\section{COLLECTEURS POUR LE 3 e FASGIGULE}

C1. Bicknell, à Bordighera (Italie), no $14 \%$.

$\times \mathbf{T}$. Chaboisseau (France), nos 130, 138.

Dr' W.-O. Focke, à Brême (Allemagne), n" 109.

$\times$ G. Genevier (France), no 148 .

F. Gravet, à Louette-Saint-Pierre (Belgique), no 102.

H. Hofmann, prof. à Grossenhain (Saxe), nos 104, 127, 129, 142, 146.

Jaquet, instituteur, à Châtel (Suisse). no 132.

Jourdes, à Verreuil, Eure (France), nos 103, 134.

F. Kretzer, prof. à Braunsweich (Allemagne), no 101.

Rv. E.-F. Linton, Salisbury (Angleterre), nos 105, 106, 107, 144.

$\times$ P.J. Müller (Alsace), $1^{\text {ns }} 115,128,150,126$ p.p.

$\times \mathbf{N}$. Boulay, prof. à Lille (France), $n^{\circ} 125$.

$\times$ Questier (France), nos 108, 110, 112, 133, 149, 126 p.p.

Dr H. Sabransky, à Sochau (Styrie), nos 136, 141, 143.

G. Sampaio, Porto (Portugal), nos 111, 116, 122, 124, 13\%, 14.

H. Sudre, prof. à Toulouse (France), nos 113, 114, 117, 118, 119, I23, 131, 135, 140.

$\times$ Timbal-Lagrave (France), nos 120, 121, 139.

Ce fascicule renferme 24 Pubus qui n'ont jamais étó distribués dans des exsiccata numérotés, et 29 numéros d'une authonticité absolue !

H. SUDRE.

\section{OBSERVATION}

Je prie les collahorateurs qui rócoltent des Rubus hybrides de vouloir bien madresser un exemplaire de chacun des parents présumés. J'accepte les esjèces non déterminées pourvu rju'clles soient bien préparées.

La préparation du Batotheca ayant lieu chaque amnece cu octobre, les envois doivent me parvenir au commencement de ce mois. 
Sect. I. - Suberecti P.-J. Müll.

$\mathrm{N}^{0} 101$ - IR. PILCA TUS W. N. var. ACUminatus Sud. - Allemagne: Braunsweich (F. Kretzer).

Var. voisine du $R$. rosulentus P.-J. Müll., mais en différant par ses feuilles caulinaires très grandes, plus régulièrement dentées, à folioles insensiblement et longuement acuminées. Diffère du $R$. opacus $\mathrm{F}$. par ses étamines plus courtes, ses fleurs roses et ses folioles inférieures subsessiles. Le calice paraît se réfléchir incomplètement.

No $12-R$. cæresiensis Sud. et Gravet. - Belgique : Louette-St-Pierre (F. Gravet).

Diffère du R. plicatıs W. N. par ses aiguillons petits, ses feuilles très pubescentes en dessous, à foliole caulinaire terminale moins large à sa base, à folioles latérales pétiolulées; par son inflorescence plus poilue, à quelques glandes tines et courtes sur les sépales et les pédicelles. Le calice se rétléchit làchement et la plante se rapproche du $R$. nilidus. Ainsi nommée de lancien pays des Ciereses, ou elle a été découverte. M. Focke la rattache à son $R$. cmmobius, mais celui-ci a des étamines plus longues, des feuilles un peu discolores et n'est pas glanduleux.

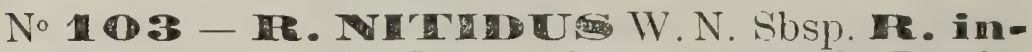
tegr-ibasis P.-J. Müll. - Orne : forèt du Perche (Jourdes).

Bien que les feuilles caulinaires aient été cueillies trop tòt et ne soient qu'imparfaitement développées, la plante est très typique.

No $104-$ R. SENTCGODES Kohler. Saxe (H. Hofmann).

Sect. II. - Silvatici P.-J. Müll.

Gr. a. Grati Sud.

No 105 - RE. CA PRTINITOLEUS Wh. - Angleterre : Shirley (E.-F. Linton).

No 106 - Re. VUCGATES W. et N. Shsp. 1F almori Lindg. - Angleterre: Bournemouth (E.-F. Linton). 
Dans mes "Observations sur sct of British Rubi", je laai rapproché de mon R. brachylhyrsus. Maintenant que j’ai pu étudier toute la centurie de M. Linton, il mo parait préférable de le subordonner an R. rulgar is W. N., à cause de ses glandes à peu près nulles et de ses pétales ovales. Le calice paraît se rétléchir imparfaitement.

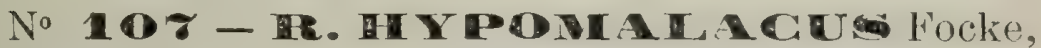
Subsp. He. mucronationmis sud. - Angleterre (E.-F. Linton).

Diffère du R. Typomalacus Focke surtont par ses turions plus anguleux, à aiguillons plus comprimés, et par la forme de ses folioles, qui sont plus élargies, nettement cuspidées et un pen plus superficiellement dentées. Ses anthères sont glabres. Ce caractère, la glabrescence du turion et la rareté de ses glandes, le distinguent du R. mucronifer sud. (R. mucronatus Blox. non ser.), auquel il ressemble par la forme de ses folioles, mais qui est plus hétéracanthe. Très fertile.

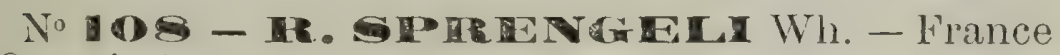
(Questier).

La plante distribuée est le $R$. nemocharis Müll. et Lef. !, qui a les feuilles caulinaires souvent 5-foliolées et les ai ruillons en général moins courbés que dans la plante d'Allemagne. Il me semble qu'il n'y a pas lieu de la distinguer du type, mème ì titre de variété.

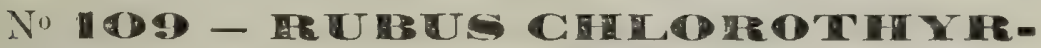

SUS Focke! - Allemagne (Dr IV.-(). locke).

Gr. b. Euvirescentes Gen.

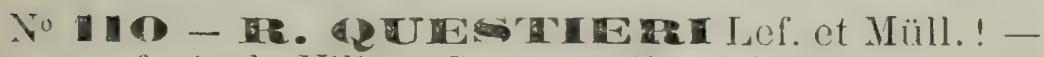
F'ance, forèt de Villers-Cotterets (Questier).

spécimens authentiques.

No 11 - IR. Gampaianus b́ud. - Portugal (G. Sampaio).

Apparemment sous-espece du R. rhombifolius Wh. En diffère par ses folioles phos irrégulicenent dentées, les terminales moins nettrment rhombées ot moins acuminées; fall sos aiguillons folus complós,

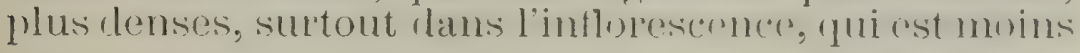


feuillée et à pédoncules plus étalés, enfin pàr ses organes floraux pàles. Se rapproche aussi des $R$. carpinifolius Wh. N. mais s'en éloigne par ses axes florifères plus velus, ses sépales réfléchis, non aculéolés. Il sert de transition entre ces deux espèces. Son inflorescence très armée l'éloigne du $R$. leucander Focke.

No 112 - IR. PYMAMIDALIS Kalt. France : Valois (Quertier).

\section{No 11 is - Re. glaucoxylon Sud. - France: Tarn (H. Sudre).}

Diffère du R. pyramidalis Kalt. par ses turions glabres et glaucescents, ses aiguillons plus denses, plus inégaux, moins élargis à leur base; ses feuilles plus finement dentées, à dents non étalées, à foliole caul. terminale largement ovale, un peu échancrée, à pétiolule égalant le $1 / 8$ de sa hauteur; par ses rameaux munis d'aiguillons petits, inégaux; son inflorescence moins longuement hérissée, manifestement glanduleuse ; ses sépales tomenteux-verdàties, non ou courtement poilus. AC. dans la partie septentrionale du Tarn.

\section{$\mathrm{N}^{\circ} 112$ - R. CGILECHTENDALI Wh. - Tarn : Carmaux (H. Sudre).}

Weihe et Nées attribuent à leur plante des pétales blancs et des étamines roses; dans la forme distribuée les pétales sont rosés et les étamines plus pâles, mais je n'attache pas grande importance à cette légère différence. Sur le même turion, à côté de feuilles $\dot{a}$ foliole caulinaire terminale obovale et absolument conforme à celle de la planche des Rubi germanici, s'en trouvent quelques-unes d'elliptiques. Il me semble que la plante du Tarn doit ètre plus rapprochée de la plante type de Mennighüffen que celles de l'Oise, que je distribuerai prochainement, et surtout d'Angleterre que l'on rattache à la même espèce.

\section{Gr. c. Discoloroides Gen.}

No 15 - TR. incarnadus P.-J. Müll.! Alsace (Müller).

Le R. incarnatus Müll., dont je distribue des spécimens authentiques, est intermédiaire entre le $k$. villicaulis Koehl. et le R. Godroni Lec. et Lamt. Le $R$. ste- 
reacanthus P.-J. Müll., qui est (salt. ex pte) le $k$. villicaulis B. incarnatus Focke ap. Asch. et Gr. Syn. VI $p . \breve{j} 16$, est pour moi une simple variété du type $R$. villicaulis. Il en est de même des spécimens que j'ai recus de M. Focke sous le noin de $R$. villicaulis sbsp. incarnatus (non P.-J. Müll.).

No 16 - TR. incarnatus P.-J. Müll. var. subincertus (G. Samp.). - Portugal (G. Sampaio).

Diffère du type par sa denticulation plus grosse et plus irrégulière, ses aiguillons plus espacés, son inflorescence peu poilue et ses sépales plus verdâtres. Se rapproche un peu du $R$. affinis W. et $\mathrm{N}$.

No11 - R. VILLICAULIS Kohl. Sbsp. 1R. Ornatus Sud. - France : Tarn (H. Sudre).

Diffère du $R$. villicaulis Koehl. par ses turions plus velus, à aiguillons plus denses et plus coniques; par ses folioles caulinaires terminales plus larges, et sa denticulation plus superficielle; par son inflorescence courte, large et lâche, à pétales étroits. Il est souvent presque entièrement virescent. Ses aiguillons rapprochés rappellent ceux du R. fagicola de Martr. Commun dans l'Albigeois; précoce et fertile.

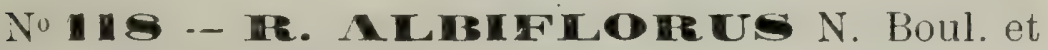
Luc. - Puy-de-Dôme (H. Sudre).

Cette plante est normalement discolore, mais comme elle croît le plus souvent dans des lieux très ombragés elle devient subvirescente. Le turion est visiblement poila et non glabrescent comme l'indique la Flore de France. Certaines formes que j'ai recues de Siane-et-Loire se rapprochent beaucoup du $R$. flacciclus P.-J. Mül. et il est possible que les $R$. albiflorus et flaccidus ne soient que deux variétés extrêmes d'une mème espéce. Elles ont beancoup de caractères communs: feuillage flasque, inflorescence très hérissée, pollen imparfait, etc. Le $R$. flaceidus de P.-J. Müll. (non Bacnit\% Herb. cur. 8.5)t !) diffëre de la plante distribuée par l'absence de glandes, le feuillage plus discolore, la foliole caulinaire terminale plus petite, un pell oborée et moins échancrée, son inflorescence plus ample, ses aiguillons plus forts. Il semble se placer dans le voisinage du $R$. pubcscens Whe, mais pentètre n'est-il qu'une variété aprique du le. albifloris? 


\section{$-42-$}

Sect. III. - Discolores P.-J. Müll.

Gr. a. Gypsocaulon P.-J. Müll.

No 119 - 18. ULMIEOLIUS Schott. $M i$ crog. R. contractifolius Sud. var. disjunctifolius (Boul. et Let. ex parte). - Tarn : Albi (H. Sudre).

La forme très étroite des folioles caulinaires terminales n'est pas due à la station; dans la même localité croît le $R$. dilatatifolius Sud. dont les folioles sont à peu près orbiculaires.

\section{$\mathrm{N}^{0} \mathbf{2 0}$-- R. UCMITOLIUSSbsp. TR. ani-}

sodon Sud, var. peliolulatus (Mül. et Timb.) 1 Toulouse (Timbal).

Foliole caulinaire terminale subobovée-rhombiforme, entière, atténuée en pointe, très longuement pétiolulée ; dents inégalement incisées-anguleuses; aiguillons pétiolaires courts, falqués; panicule plus ou moins inerme. Forme cérinostyle. La plante est en partie atteinte de pilosisme; l'axe de l'inflorescence n'est pas normalement tomenteux-tloconneux.

$\mathrm{N}^{\circ} \geq 1-\times$ R. PLATYPETALUS P.-J. Müll. et Timb.; R. ulmifolius $X$ lacertosus sud. - HauteGaronne : Grizolles (Timbal).

La plante de St-Urcisse (Tarn) que Timbal avait rapportée au $R$. platypelalus M. et T. et qui est décrite dans la Florule du Tarn est quelque peu différente du type primitif, que je distribue : toutefois il est possible, étant donné le polymorphisme du $R$. ulmifolius, que cette plante ait la même origine que celle-ci et ne soit pas un hybride compliqué ainsi que je l'ai pensé autrefois (Rév. Rub. Hb. Tarn). Je groupe sous le nom de $R$. platypetalus tous les hybrides de la forme: ulmifolius $\times$ lacerlosus. Ce $n^{0}$ représente une forme moyenne de cet hybride, où l'influence du $R$. ulmifolius (vulgatus) est prédominante; cette forme n'est pas rare aux environs d'Albi et dars le Tarn, où les parents abondent. J'espère que les circonstances me permettront de récolter le $R$. Bosquetianus Timb. et M., qui est un $R$. lacertosus $\times$ ulmifolius.

No $\mathbf{2} \mathbf{2}$ - Me. ellipticifolius Sud. - PortuSud. - Portugal : Porto (G. Sampaio).

'Turion glauque 1 caractère que je n'avais pas noté 


\section{$-43-$}

mais que possèdent bien mes spécimens des Pyrénées. La plante est ì rapprocher du R. Godroni Lec: et Laint. et non du $R$. hedycarpus Focke. C'est peut-ètre une espèce de premier ordre?

Gr. b. Hedycarpi (Focke).

No 23 - Re. CUSRTDIFER Mül. et Lef. var. rhombophyllus sud. - Tarn (H. Sudre).

Diffère du type cuspidifer par ses folioles caulinaires terminales moins larges, rhomboïdes, aiguës ou brièvement et insensiblement acuminées, entières à la base, son inflorescence plus dense, moins feuillée. Par la forme de ses folioles, cette variété se rapproche un peu du $R$. amiantinus Focke (R. lepidus Müi!.); mais elle en diffère par sa denticulation plus irrégulière, son turion plus pubescent et surtont par ses pétales grands et larges, plus pâles.

No $\mathbf{2}$ \& - IR. PUDEGCANS Wh. Sbsp. R. aduncispinus Sud. - Portugal : Povoa de Lanhoso (4. Sampaio).

Folioles assez finement dentées, la caulinaire terminale obovale, entière, cuspidée. Aiguillons pétiolaires et raméaux la plupart vivement crochus. Inflorescence maigrement hérissée, à pédicelles souvent fasciculés dès la base et un peu ascendants. N'est pas rare dans l'ouest de la France: Loire-Inf., Maine-et-Loire, etc.

No 125 - Re. PTRCIRTES P.-J. Müll.! France: Vosges (N. Boulay).

M. Focke rattache le R. procerus Mïll. ì son R. leucander (ap). Asch. et (rro). J'estime, avec M. l'abbé N. Boulay, que les R. robustus Müll. et procerus Müll. ne sont que deux variétés d'un mème type. Dans le $\mathrm{n}^{\circ} 69$ clu:Batolleca jai arlopté le nom de $R$. robuslus, qui date de 185.9, pour désigner re grompe spécitique; nais comme il existe un $R$. robustus Presl. antérieur à celui de Müller, lespéce doit porter le nom de R. procerus. Le no $6 !$ doit ètre envisagé comme une variété de celui-ci : $R$. lnocemes I'.-J. Nïll. val. robustus.

Gr. c. Candicantes Focke.

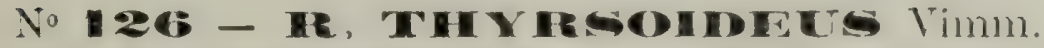

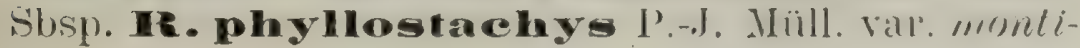


genus (P.-J. Müll.)! - Alsace, Marne et Valois (Müller, Levent et Questier).

Turion vigoureux, canaliculé, glabrescent, à aiguillons forts; pétiole à aiguillons robustes, falqués; feuilles grossièrement et irrégulièrement dentées; foliole terminale, ovale, échancrée, acuminée; rameau à aiguillons très forts, à base large, falqués, devenant même assez souvent crochus dans la région de l'intlorescence, dont les rameaux sont un peu ascendanls; pétales ovales; pollen très imparfait.

No 27 - TE. THYTESOIDEUS Vimm. Sbsp. Fe. candicans Wh. - Saxe (H. Hofmann).

La plante distribuée est une forme moyenne, pouvant servir de type.

$\mathrm{N}^{\circ} \mathbf{2 8}$ - He. candicans Wh. var. excelsior (P.-J. Müll. !) - Alsace (Müller).

Cette variété est caractérisée par des turions glabres, à angles obtus, à faces planes ou peu excavées; des folioles terminales elliptiques, acuminćes, à base moins large que dans la var. courctatus, assez souvent entières; les folioles raméales terminales sont étroiles et acuminées, très irrégulièremont dentées; l'inflorescence est vaste, peu armée, plus ou moins feuillée, à pédoncules moyens étalés, très multiflores; les pétales sont ovales, blanes ou un peu rosés.

No 29 - Re. candicans Wh. var. excelsior (P.-J. Müll.) - Saxe (H. Hofmann).

\section{Gr.d - Subtomentosi Sud.}

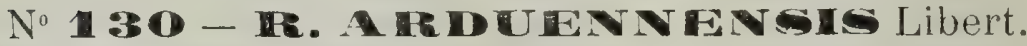
Sbsp. Re. obtusatu $\rightarrow$ P.-J. Müll.! - France, Vienne (Chaboisseau).

spécimens authentiques! - J'avais d'abord pris cette plante pour un hybride; mais d'après une note de Chaboisseau jointe à la centurie distribuée, le $k$ obtusatus n'est pas rare dans la région granitique de la Vienne et du Limousin. D'autre part, j'ai maintenant la conviction que mon $R$. anglesensis n'en est pas sensiblement différent, ainsi qu'on pourra en juger par les spécimens du numéro suirant. Le $R$. obluscilus a donc bien les apparences d une forme pure, qui se place dans le voisinage du $R$. arduennensis Lib. 
No I31 - IR. ATEDUENNENGIS lib. Sbsp. Re. obtusatus P.-J. Mülli. - France : Tarn (H. Sudre).

Fructifie bien; a le turion plus nettement canaliculé que la plante de la Vienne, mais ne me paraît pas en différer. Le $R$. consobrinus Sud. croît dans la montagne d'Anglès, mais je ne crois pas que le $\mathrm{n}^{0} 131$ en dérive. Est très répandu dans toute la montagne d’Anglés.

Sect. IV - Appendiculati Gen.

Gr. b - Vestiti Focke

No I3:- IR. VESTRTUS Wh. - Suisse (Jaquet).

No 133 - TR. ADSCITUS Gen. - France : Valois (Questier).

No $13 \mathbf{3}-\times$ R. TOUSSAINTI Sud.; $R$. adscitus $\times$ ulmifolius. - France, Orne (Jourdes).

Rappelle le $R$. adscitus Gen. par la pilosité des turions, de la face inférieure des feuilles et des axes florifères, le $R$. ulmifolius Schott par ses turions glaucescents et ses feuilles d'un vert sombre en dessus.

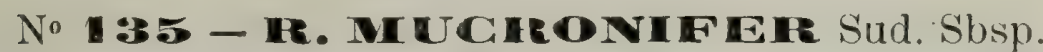
Re. parcepilosus Sud. - Hautes-Pyrénées (H. Sudre).

No 36 - Ie. COTEMINNT Blox. Susp. Re. Gemlii Focke - Styrie (Sabransky).

La forme distribuée, répandue en Styrie, en Bavière, en Bohême, en Moravie, etc., est plus glanduleuse, plus robuste et plus hétéracanthe que le type de Gremli, mais des intermédiaires insensibles semblent la rattacher directement à ce type. Diffère des R. Colcmanni Blox. surtout par ses aiguillons moins forts et moins courbés dans l'inflorescence, ses étamines moins longues et ses folioles terminales insensiblement acuminées.

(Xr. c. Radula Focke.

No 137 - TR. GENEVHEIRI Bor. Micr. R. brigantinus G. Samp. ! - Portugal (G. Sampaio). 
Diffère dı R. Genevieri Bor. par ses turions subarrondis, ses aiguillons un peu coniques, ses dents plus fines, son intlorescence plus étroite, plus dense, peu armée et à glandes courtes. Se rapproche de certaines formes du groupe du R. macrostachys P.-J. Müll., f:n particulier du R. rubellus Müll. An R. Genevier $i$ X?

No 138 - He. GENETIERI Bor. Sbsp. R. discerptus Müll.! - France : Vienne (Chaboisseau).

Spécimens authentiques! - Le nom de $R$. echinatus Lindl. paraît avoir été appliqué à des formes diverses; il me semble préférable d'adopter celui de discerptus dont la description est très précise et dont l'attribution est certaine. - J'ai vu des forêts de l'Oise des intermédiaires embarrassants qui ine portent à subordonner cette plante au $R$. Genevieri Bor.

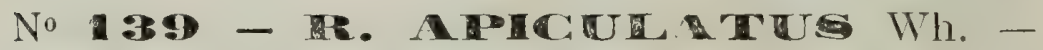
France: Hte-Garonne (Timbal).

La plante distribuée semble avoir très exactement tous les caractères de celle de Weihe et Nees; je l'ai retrouvée, après Timbal, dans les bois d'Aufréri, près de Toulouse. Elle croît dans un endroit très ombragé et est moins discolore que d'habitude.

$\mathrm{N}^{\circ}$ A - DE. MICA God. Microg. R. micantiformis Sud. - France: Tarn (H. Sudre).

Diffère du R. micans God. par ses turions glabres et glauques, dressés; sa foliole caul. terminale largement ovale ou suborbiculaire, en cœur, brusquement et brièvement acuminée.

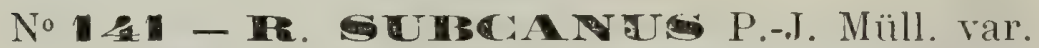
supinus (Sabrs. p. sp. O.-b-Z; 1905, n 8). - Styrie or. (Sabransky).

Très voisin du type de Müller; toutefois, turion plus nettement glauque ; feuilles caulinaires toutes ternées, plus pubescentes en dessous; calice aculéolé; styles verdâtres.

No 12 - He. GHANULATUS M. et L. Sbsp. Fe.miamiensis Hofm. - Saxe (Hofmann).

Le rameau est très hétéracanthe comme dans les vrais $R$. Rachlae, mais le turion n'a que quelques 
rares aiguillons tuberculiformes. Malgré cela je ne crois pas que la plante puisse se metre dans les $R$. silvatici P.-J. Müll., où se place le $R$. amygdalanthus Focke. Le $R$. misniensis Hofin. est intermédiaire entre le $R$. granulatus M. et L. et le $R$. silesiacus Wh.

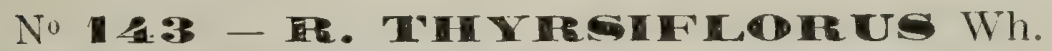
Sbsp. It chloranthus (Sabrs, ! pr. var. O.-b.-Z., 190 o, nº 8 - Styrie (Sabransky).

Remarquable par ses turions glabres et glaucescents, son inflorescence feuillée, à glandes très courtes, et la brièveté de ses étamines.

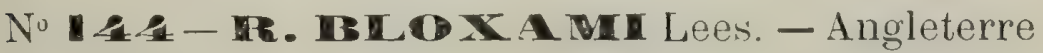
(E. F. Linton).

La plante a des turions très velus; elle me paraît plus rapprochée du $R$. pallidus Wh. que du $R$. thyrsiflorus Wh., auquel M. Focke l'identifie. Elle est également distincte des $R$. condensatus et rhenamus P.-J. Müll. - Se rapproche du $R$. Menkei Wh. par le $R$. multifidus B. et M.

No 15 - TR. Honniquosi G. Samp. - Portugal (G. Sampaio).

Intermédiaire entre $R$. Menkei Wh. et $R$. mucronifer sud. Diffère de ce dernier par ses turions plus scabres, ses feuilles :3-nées, ses anthères non poilues. - "Très fréquent dans toutes les montagnes du nord du Portugal "(G. Sampaio, ni litt.)

Gr. e. Hystrices Focke

No A6 - Re. IEQEHCETEX Wh. - Saxe (H. Hofmann).

Gr. f. Glandulosi P.-J. Müll.

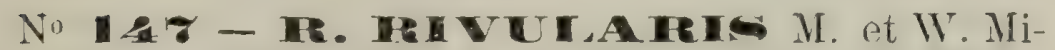
cron. $\mathrm{R}$. horridipes sud. - Italie: Ligurie (C. Bicknell).

Voisin du R. horridulus I'.J. Müll. En diffëre par ses turions glanques, ses feuilles moins finement dentées, velues en dessous, les caul. j-nées; par sa foliole caul. terminale nettement émarrinée; son in- 


\section{$-48-$}

florescence plus allongée, plus multiflore, plus armée et plus feuillée; ses styles pâles et ses carpelles glabres.

No 148 - M. TERETICAULIS Müll. var. breviglandulosus Sud. - France : Maine-et-Loire (Genevier).

Diffère du type par ses folioles caul. terminales plus courtes, brièvement acuminées, son inflorescence paucitlore, dépassant peu les teuilles, munie de glandes pâles et courtes.

$$
\text { Sect. V - Triviales P.-J. Müll. }
$$

No $19-X$ R. IMPLEXUS Lef.; R. rudis $\times$ cosius N. Boul. - France : Oise (Questier).

$$
\text { Sbg, Idæobatus Focke }
$$

No 150 - R. ID AEUS L. - Alsace (Müller).

\section{H. SUDRE.}

Novembre 1905.

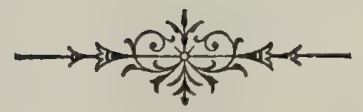




Batotheca

Fasi IV

Myor 



\section{BATOTHECA EUROPAA}

PUBIIE PAR

H. SUDRE

Professeur à l'Ecole normale de Toulouse (France)

Allées Saint-Michel, 19

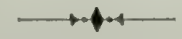

FASCICULE IV - 1906

Parait par fascicules annuels de 500 numéros. I.es collaborateurs qui fournissent 5 numéros convenus d'avance et préparés à 2ố parts, recoivent un fascicule en échange. Chaque exemplaire doit comprenure, autant que possible, deux rameaux, l'un florifère et l'autre fructifère, plusieur's feuilles caulinaires et quelques pétales préparés à part. Les espèces déjà distribuées ne sont admises que si elles diffèrent quelque peu des formes publiées.

\section{Prix du fascicule : 25 fr.}

\section{एक \\ COLLEGTEURS POUR LE $4^{\circ}$ FASGICULE}

$\times \mathbf{N}$. Boulay, prof. â Lille (Nord), nº 164 .

Di: Bouly de Losdain à Dankerque (Nord), $n^{0 ;} 158,169,186$.

Chanoine Chenon (Sarthe), no 183.

Coilliot. au Mans (Sartle), no 172.

K. Friderichsen à Hover (Schleswig), no 192.

F. Gravet, ¿ Louette-St-Pierre (Belgique), ns 177, 178, 179, 191, 196.

A. Henry, au Mans (Sarthe), nos 155, 165, 16\%, 170.

H. Hofmann, prof. a Grossenhain (Saxe), nos 152, 15', 187, 189, $195,198$.

Jaquet, à Châtel (Suisse), no 163.

D' H. Kinscher, à Peterwit\% (Silésie), no 184.

Rv. E. F. Linton, Salisbury (Angleterre), n 168.

$\times$ P.-J. Mủller. Wissembourg (Alsace), nos 151, 200.

$\times$ Questier. Thiury-cri-Valois (France), nos 156, 175, 180.

D. H. Sabransky. Sorliau (Sirric). $11^{0 *}, 173,188,190,193,194,167$.

G. Sampaio, Acad. polyt., Poito (Portugal), no 182.

spribille, prof. à Holiensalza (Posen), 1 $^{\text {ns }} 176.185$.

H. Sudre, Hol. à Toulouse, 11ut 153, 157, 159, 161, 162, 1C6, 171, 181, 199.

$\times$ Timbal-Lagrave, Toulouse, no3 $160,1 \% 4$.

Ce fascicule renferme 30 Rubur rui n’ont jamais été rlistribués dans des exsiccata ıumérotés, et jó numéros dont l'authenticité est absolue !

H. SUDRE. 
Sect. I. - Suberecti P.-J. Müll.

No 151 - TE. NHTDES W. et N. var. DIFARicatus (P.-J. Müll. !). - Alsace (Müller).

Spécimens authentiques ! La centurie de Müller n'ayant que quelques feuilles caulinaires, j'en ai reproduit deux par autographie.

No 152 - Re. NITIDES W. et N. var. Divaricatus (P.-J. Müll.) - Saxe (H. Hofmann).

Forme aprique bien développée, à aiguillons un peu plus denses et plus courbés que dans le $n^{0} 151$, et se rapprochant de la var. hamulosus (Lef. et Müll.).

Sect. II. - Silvatici P.-J. Müll.

a. Grati Sud.

No153- R. TRETAGUS Sud. - France : Tarn (H. Sudrè).

Diffère du $R$. gralus Focke par ses turions glabres, ses feuilles glabrescentes en dessous, moins grossièrement dentées, son inflorescence non poilue, courte, ample, et ses pétales très étroits.

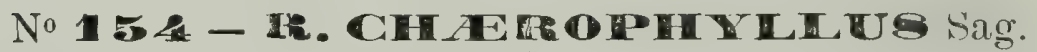
et Sch. - Saxe(H. Hofmann).

Le $R$. charophylloides Spribille, identifié par M. Focke au $R$. chcerophyllus, appartient aux $R$. vestiti et est voisin de mon $R$. hebecaulis.

No $155-X$ R. GRANIIBRACTEATUS Sud. ; R. Questieri X Fuscus. - Sarthe, Le Mans (Henry).

Faciès du R. Questieri Lef. et M. qu'il rappelle par la forme de ses tolioles, son inflorescence très feuillée et ses fleurs roses; mais turion pubescent, à quelques aiguillons tuberculiformes ; rameau manifestement hétéracanthe, inflorescence glanduleuse comme chez le $R$. fuscus Wh. Stérile en 1905, a fructifié partiellement en 1906. 
b. Euvirescentes Gen.

No $56-R$. eglandulosus M. et Lef. ! - Aisne (Questier).

Fructifie. Intermédiaire entre $R$. Questieri L. et M. et $R$. macrophyllus $\mathrm{W}$. et $\mathrm{N}$. Dérive peut-ètre du $1^{\mathrm{e}}$ par croisement. Jusqu'ici spécial au Valois (Aisne et Oise).

\section{c. Discoloroides Gen.}

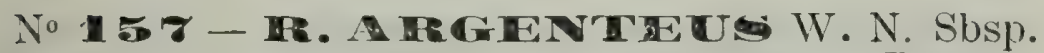
Re. inceanatus Müll. var. Conformis. - Tarn-etGaronne (H. Sudre).

Le R. incarnalus Müll., d'abord subordonné au $R$. villicaulis (Bat. eur. no 115), me paraît plus rapproché du $R$. cirgenteus IV. N. Le $n^{0} 155$ diffère du $n^{0} 115$ parses folioles caul. terminales à base moins large, ses dents moins divariquées, ses aiguillons moins courbés sur le rameau, ses carpelles velus.

No $158-\times$ R. DREPANOSTACHYS Sud, R. LESDaini $X$ ulmifolius. -- Nord (B. de Lesdain).

Le $n^{\circ} 195$ des Rubi gallici, portant le nom de $R$. ulmifolius $\times$ foliosus, est selon moi une forme pure du grompe du R. alterniflorus M. et L., que j'ai appelée R. Lesdaini (ap. Gdg. Nov. consp. p. 139). Le no 157 a le faciès du $n^{\circ} 195$ mais en diffère par ses turions glanques, ses feuilles plus discolores, ses glandes courtes et peu apparentes, son inflorescence lâche, très armée ; il est stérile.

No 59 - He. ATTERENHLORUA M. et $\mathrm{L}$. s. Sbsp). R. phyllanthoides Sud. - 'Tarn, monts de Lacaune (H. Sudre).

Differe du R. allerniflorus M. et L. par sa foliole caulinaire terminale largement ovale, échancrée, acuminée ; son inflorescence allongée, abondamment feuillée jusqu'au sommet, à pédoncules ascendants, couverts de nombreuses glandes courtes et peu apparentes. - Assez communi dans la région montagneuse du Tarn se rencontre aussi dans le l'inistère. 
Sect. III. - Discolores P.-J. Müll.

a. Gypsocaulon Müll.

No 60 - R. ULMIFOLIUS Schott Micrg. He. Serriculatus Rip. var. Congeneratus (Müll. et Timb.)! - Toulouse (Timbal).

Diffère du type $R$. serriculatus Rip. par sa denticulation un peu irrégulière et son inflorescence subinerme.

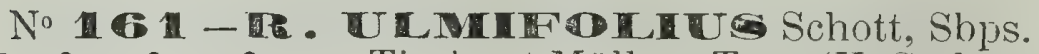
He. insignitus Timb. et Müll. - Tarn (H. Sudre)'

Diffère du type $R$. insignilus par ses styles pàles; se rapproche un peu de la var. dispalatus Sud. Rub. Pyr. p. 10.

\section{b. Hedycarpi (Focke).}

No 1 - Th. difficilis Sud. - Htes-Pyrénées: Cazeaux-Débat (H. Sudre).

Cette espèce, jusqu'ici spéciale aux Pyrénées centrales, est à rapprocher du $R$. cuspidifer Mül. et Lef. La forme de ses folioles terminales, son inflorescence grande, nue, à pédoncules élalés, ses pétales étroits, ses organes floraux entièrement roses et son pollen inparfait l'en distinguent suffisamment. Certains rameaux, cueillis dans une haie très fournie, sont presque virescents.

No 63 - PE. PR⿴囗十 SANGulus (Gremli) - Suisse (Jaquet!

Plus grêle que IR. procerıs, feuilles à poils apprimés en dessus; folioles moins larges.

c. Candicantes Focke.

No 164 - TR. THYTRGOHDEUS Wimm. Sbsp. Re. phyllostachys P.-J. Müll. - Vosges (N. Boulay). 
C'est la mème plante que le $\mathrm{n}^{0} 4$ des Ronces rosgiennes, déterminé par Müller. L’abbé Boulay, qui consilérait le $R$. phyllostachys d'Alsace comme un $R$. lllyrsoideus $\times$ macrophyllus, rattachait ce $n^{0} 4$ an $R$. cundicans comme forme. J'estime que l'interprétation de ce batologue est erronée et que la plante de Rambervillers est à peu près identique à celle d'Alsace. Voici lu reste la note de Müller: "N’étaient les flenrs rosées, je le prendrais assez volontiers pour le R. pluyllostachys, dont il offre les principaux caractères $"$.

\section{d. Subtomentosi Sud.}

No 165 - Th. Linkiamug Ser. - Sarthe (Henry).

Paraît mal fructifier et dérive apparemment du k. tomentosus Sbsp. Lloydianus par croisement. Naturalisé sur un grand nombre de points en France.

$$
\text { Sect. IV. - Appendiculati Gen. }
$$

a. Tomentosi Wirtg.

No 16G - TE. TONAENTOSED Borckh. T'oulouse (H. Sudre).

A été cueilli en pleine forêt.

b. Vestiti Focke.

\section{No 167 - RE. VEGTITUE Wh. Sbsp.} IR. leucotriehus Sud. - Sarthe (Henry).

Diffère du $R$. vestilus Wh. par ses aiguillons à peu près égaux, ses glandes rares et courtes, ses feuilles ininces, glabres ou à poils rares en dessus, à foliole caul. terminale obovale, entière, cuspidée ; son intlorescence i villosité blanche, longue et dense. Ce $n^{\circ}$ a mal fructifié en 1906, probablement à cause de la sécheresse exceptionnelle de l'été. La même plante a bien fructifié à l'Epau (Sarthe) cette année, ainsi que l'a observé M. Henry.

No 163 - IR. VESTTE- Wh. Sbsp. re. leveoteichus sud. var. Subeglandulosus. - Angleterre, Edmondsham (E. F. Linton). 
Diffère du précédent par son inflorescence à glandes à peu près nulles, ses turions plus anguleux, moins hérissés. Il n'est pour ainsi dire pas héréracanthe et semble appartenir aux $R$. silvatici; il a dû pousser à l'ombre car, si certains rameaux sont assez nettement discolores, d'autres sont entièrement virescents.

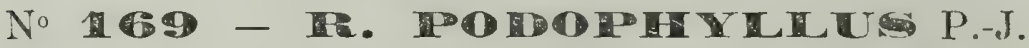
Müll. var. Lutetranus Sud. - Seine (B. de Lesdain).

Turion glaucescent, à glandes nombreuses; feuilles plus discolores que dans les autres variétés de cette espèce ; foliole caul. terminale courtement obovée, un peu échancrée, brusquement acuminée ; inflorescence ample, presque nue, peu armée, à axe très poiluhérissé, à pédoncules étalés ; pétales elliptiques ; carpelles très hérissés; pollen peu mélangé. Se rapproche du $R$. apiculatus Wh. Croît aussi dans la forèt de Rambouillet (Mlle Bélèze).

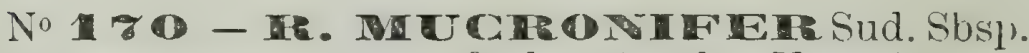
IR. conomanensis Sud. - Sarthe (Henry).

Diffère du $R$. mucronifer surtout par ses turions glauques, ses folioles obovales, bien plus étroites, ses anthères glabres.

No 1 - R. TETECAUIS Sud. Sbsp. 1R. podophylloidos ej. - Tarn (H. Sudre).

Diffère du $R$. hebecaulis par ses folioles obovales, finement dentées; est ordinairement discolore.

\section{c. Radulce Focke.}

No $\mathbf{z} \mathbf{z}-X$ R. BREviATUS Sud. et Bouv.; $\mathrm{K}$. Genevieri X ulmifolius. - Sarthe (Coilliot).

Diffère du R. Genevieri Bor. par ses turions glauques, peu hétéracanthes, son inflorescence ì glandes courtes, ses fleurs roses. Stérile.

No 1 \%:B - Fe. MIMANS God. Sbsp. Fe. heterochnous sud. var. barbatus (Sabrs.) - Styrie (Sabransky). 
Diffëre du R. heterochrous des Pyrénées par sa denticulation plus vive, son inflorescence plus allongée et très feuillée. S'éloigne du $R$. obscurus Kalt. par ses feuilles un peu discolores, et du $R$. insericatus P.-J. Müll. par ce même caractère et ses sépales étalés.

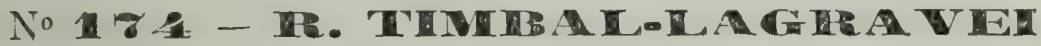 P.-J. Müll. ! - Luchon (Timbal-Lagrave).}

Plante spéciale à la vallée de Luchon et indiquée à tort par Timbal comme commune dans les Pyrénées. Le calice est nettement réfléchi et non plus ou moins redressé comme le dit la Flore de France, où cette erreur n'a pas été rectifiée malgré la remarque que j'ai faite dans mes Rubus des Pyrénées ; de plus les styles sout constamment verdattres et non d'un rose vif!

No uus (Lef. et M.) - Valois (Questier).

Spécimens tout à fait conformes aux types de Lefèvre.

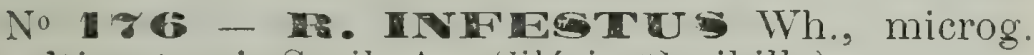
R. altipratensis Sprib. ! - Silésie (Spribille).

Diffère du $R$. infestus Wh. par ses aiguillons moins courbés, son inflorescence allongée, feuillée, ses étamines courtes. Semble fructifier partiellement. Peutêtre pourrait-on en expliquer sur place l'origine par croisement?

No I - TR. INFESTES Wh. Microg. R. holochlous sud. - Belgique (F. Gravet).

Grèle ; turion subarrondi, glabrescent ; feuilles vertes et velues en dessous, grossièrement et irrégulièrement dentées, les caul. 3-5-nées; foliole caul. terminale ovale. échancrée, insensiblement acuminée. Rameau velu, à aiguillons faibles et pâles. Infloresrence allongée, hérissée, non feuillée, à aiguillons pailes, nombreux, fins ou médiocres, déclinés ou falqués ; sépales verdàtres, étroits, acuminés, lâcheirent relevés sur le fruit ; pétales blancs, étamines blanches, dépassant les styles rerdâtres ; carpelles glabres. 
No 88 - Re. Whedous Wh. Sbsp. Fr. Follolatrs Lef. et M. - Belgique ( $\mathrm{F}$. Gravet).

A l'inflorescence un peu plus glanduleuse et les folioles un peu plus étroites que le type de Lefèrre, mais y est relié par des formes intermédiaires et je ne crois pas qu'il y ait lieu le l'en séparer.

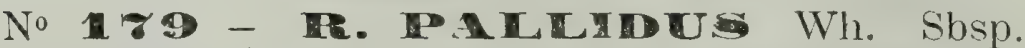
Fe. hin-sutus Wirtg. Var. PSEUDO-ERUBESCENS. Belgique (F. Gravet).

Turion très anguleux et très hétéracanthe; denticulation grossière, irrégulière; feuilles supérieures grises en dessous; foliole caul. terminale largement ovale-rhombée, acuminée; tleurs blanches, à étamines longues; carpelles velus.

Le $R$. erubescens Wirtg. est à rapprocher du $R$. foliolatus L. et M.

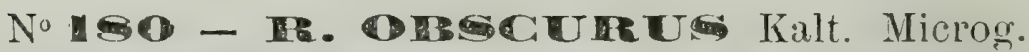
R. Sprengeliflorus Sud. - Aisne (Questier).

Je l'avais d'abord rapproché de mon $R$. erralicus (Rub. Hb. Bor. p. 67), mais l'examen de la centurie de Questier m’a convaincu que les plantes étaient réellement distinctes.

\section{d. Rudes Sud.}

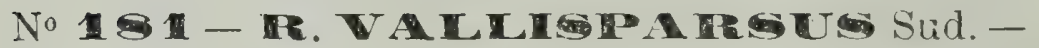
Tarn (H. Sudre).

Dans la série des $R$. rudes, le $R$. vallisparsus comprend toutes les formes faibles, à turion glaucescent, à fleurs roses et à sépales plus ou moins relevés sur le fruit. Mes R.pergratiosus, separatus, granitophilus et alnicolus rentrent dans ce groupe, entendu dans un sens très large. Le no 181 a le turion pubescent, mais est relié aux formes habituelles par de nombreux intermédiaires.

e. Hystrices Focke.

No S:- IR. TIETEGA RE PS P.-J. Müll. Microg. R. vagabundus G. Samp. - Portugal : Vieira (G. Sampaio). 
Diffère de la plante des Vosges par ses pédoncules plus courts, ramifiés dès la base, ses pédicelles plus ascendants, ses étamines plus longues et ses carpelles glabres ; il fructifie moins bien. Je subordonne au R. hebccarpus les $R$. hystrices discolores et à fleurs blanches : R. aceratispinus, infestiformis, Lapeyrousianus, doranus, spissifolius, disinnctiflorus, rubriglandulosus Sud., bavaricus Focke, etc.

No 183 - He. HETHETEL Wh. Sbsp. Te. Chemoni sud. - Sarthe (Chenon).

L'aculéation de cette plante est remarquable et, bien que les échantillons que M. le chanoine Chenon a bien voulu récolter pour ma publication soient un peu maigres, l'espèce est nettement caractérisée et facile à reconnaître; les feuilles caul. sont 3-4-5-nées.

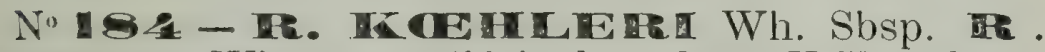
apr•icus IVimm. - Silésie, loc. class. (H. Kinscher).

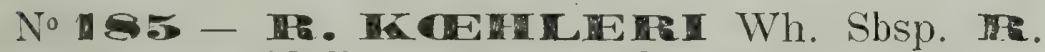
ax xicollus Müll. var. Figerti (Spribille). - Silésie (Spribille et Figert).

Plus grêle que le $R$. saxicolus Müll.; feuilles caul. en partie 3-nées, à folioles moins larges, plus vertes en dessous ; inflorescence moins développée ; sépales lâchement réfléchis.

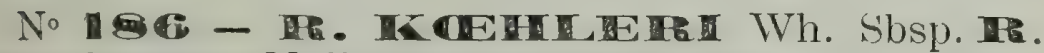
anxicolus Mill. var. PARISIENSIS Sud. - Seine-etOise (B. de Lesdain).

Feuilles amples, à dents plus grosses que dans le R. saxicolus Mïll.; foliole caul. terminale suborbiculaire, échancrée, cuspidée ; inflorescence dense. Se ripproche du $R$. horridicaulis Müll., mais a les aiguillons inoins forts et les dents plus profondes.

f. Glandulosi P.-J. Müll.

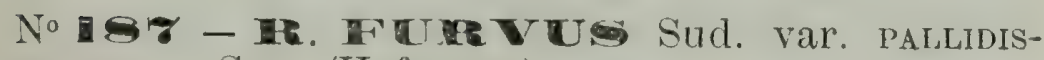
TAMINEUS. - Saxe (Hofmann). 
Le $R$. furvus a les aiguillons caulinaires nettement dilatés ‘̀ la base comme le $R$. Schleicheri, mais des fleurs roses. Le $\mathrm{n}^{0} 187$ a l'inflorescence peu armée, munie de glandes un peu brunâtres qui lui donnent l'apparence du R. Kaltenbachii Metsch.

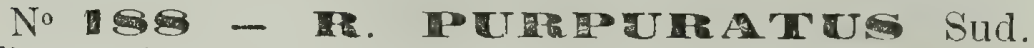
Microg. R. carneus Sabrs. - Styrie (Sabransky).

Diffère du $R$. purpuralus Sud. par ses turions un peu velus, ses dents très superficielles, même sur les feuilles raméales, son inflorescence plus armée, munie de glandes longues et denses. Intermédiaire entre R. purpuratus Sud. et $R$. prodatus Schmid. Il rappelle le R. lithophilus sud. par son inflorescence très aciculée.

No 89- TE. C IBETE Wh. - Saxe (H. Hofmann).

Le $R$. scaber, d'abord mis dans les $R$. rudes, est mieux placé dans les $R$. glandulosi, à côté du $R$. tereticaulis P.-J. Müll.

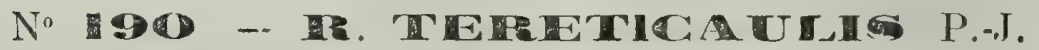
Müll. Sbsp. 直. contiulandulosms Sud. var. scYrHicus (Sabrs.) - Styrie (Sabransky).

Le $R$. curtiglandulosus diffère du R. terelicantis par ses turions plus scabres, son feuillage glaucescent, sa denticulation moins régulière, ses folioles caul. terminales de forme un peu rhombée, ses étamines plus longues. La var. scythicus diffère du type par ses axes florifères plus manifestement poilus-hérissés et son inflorescence plus aciculée. Se rapproche du R. serpens Wh.

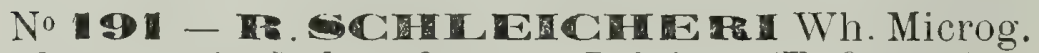
R. louettensis Sud. et Grav. - Belgique (F. Gravet).

Turion velu, non glauque; feuilles 3-5-nées, à dents peu profondes, la plupart simples ; foliole caul. terminale étroitement elliptique-obovale, entière à la base, longuement acuminée; inflorescence lâche, hérissée, presque inerme, à glandes fines et peu apparentes, étamines courtes, carpelles grabres. 
No 192 - IE. SRERTNG Wh. var. PURIPLLvis. - Schleswig (K. Friderichsen).

C'est à peu près le type du $R$. serpens tel que je le comprends. Ici le pollen présente quelques grains atrophiés, mais cela peut être dù à la station de la plante. Ce no destiné aux Rubi gullici de MM. Boulay et Bouly de Lesdain, avait aussi été appelé $R$. serpens par l'abbé Boulay.

No 193 - IR. HERERS Wh. Microg. R. longisepalus P.-J. Müll. var. scotophilcs (Halacsy). - Styrie (Sabransky).

Diffère du $R$. longiscpalus Müll. par ses folioles plus acuminées, son inflorescence plus allongée et plus feuillée.

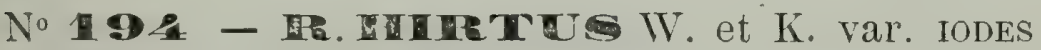
(N. Boul.) - Styrie (Sabransky).

La var. iodes ne diffère du type que par sa denticulation plus superficielle et ses carpelles glabres. Les sépales sont assez souvent diversement étalés.

No 195 - F. MRTTES W. et K. Sbsp. TR. Meneymicus G. Br. var. pubescens. - Bohème (H. Hofmann).

Remarquable parses turions et ses rameaux velus, sa denticulation très fine, ses pédoncules très étalés. Se rapproche du $R$. nigricatus Lef. et M.

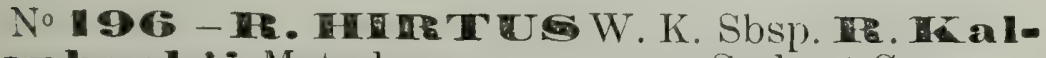
tenbachii Metsch var. ANOPLON Sud. et Grav. Belgique (F. Gravet).

Diffère de la plante de Wirtgen (Hb. $\cdot u b \cdot v h . \mathrm{n}^{\text {os }} 92 \mathrm{et}$ 42 bis) qui, d'après M. Focke, correspond au $R$. Fallenbrehii Metsch, par son inflorescence inerme, plus allongée, moins lâche, à pédoncules moins longs, plus ascendants, et par ses étamines plus longues.

No 198 - Ue. HERTES W. et K. Micrg. R. pectinatus sud. et Grav. var. HiRTissimus (Sabrs.) Styrie orient. (Sabransky). 
Diffère du R. pectinatus par ses feuilles plus manifestement velues en dessous, ses folioles acuminées, son inflorescence plus développée et plus armée, et ses styles pàles. Le $R$. erythrostachys Sabrs. $p . p$. (R. gracilis Hol.) est voisin des $R$. stellatiflorus P.-J. Müll., longipes N. Boul., longistylus Borb. et autres que je subordonne à mon $R$. interruptus Rub. Pyr. 28 .

Sect. V. - Triviales P.-J. Müll.

\footnotetext{
No $98-X$ R. HOHMANNI Sud. ; R. SENTICosus $X$ cesius. - Saxe (Hofmann).
}

Rappelle le R. senticosus Kœhl. par ses turions glabres, ses feuilles caul. en grande partie 5-nées, glabres en dessus, son inflorescence courte, norı poilue, à pédoncules très étalés, munie d'aiguillons très forts. Ce dernier caractère l'éloigne des hybrides de la forme $R$. thyrsoideus $\times$ coesius. Stérile ou peu fertile.

No $\mathbf{9 9}-X$ CHATEAUI Sud.; R. CæSiUs $X$ GILLoti. - Cantal (H. Sudre).

No $200-X$ R. VIRGULTORUM P.-J. Müll.! R. CANDiCANS $\times$ CÆsius. - Alsace (Müller).

La synonymie de cet hybride est très compliquée et je n’ai fait que l'ébaucher.

\section{H. SUDRE.}

Novembre 1906. 


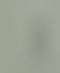
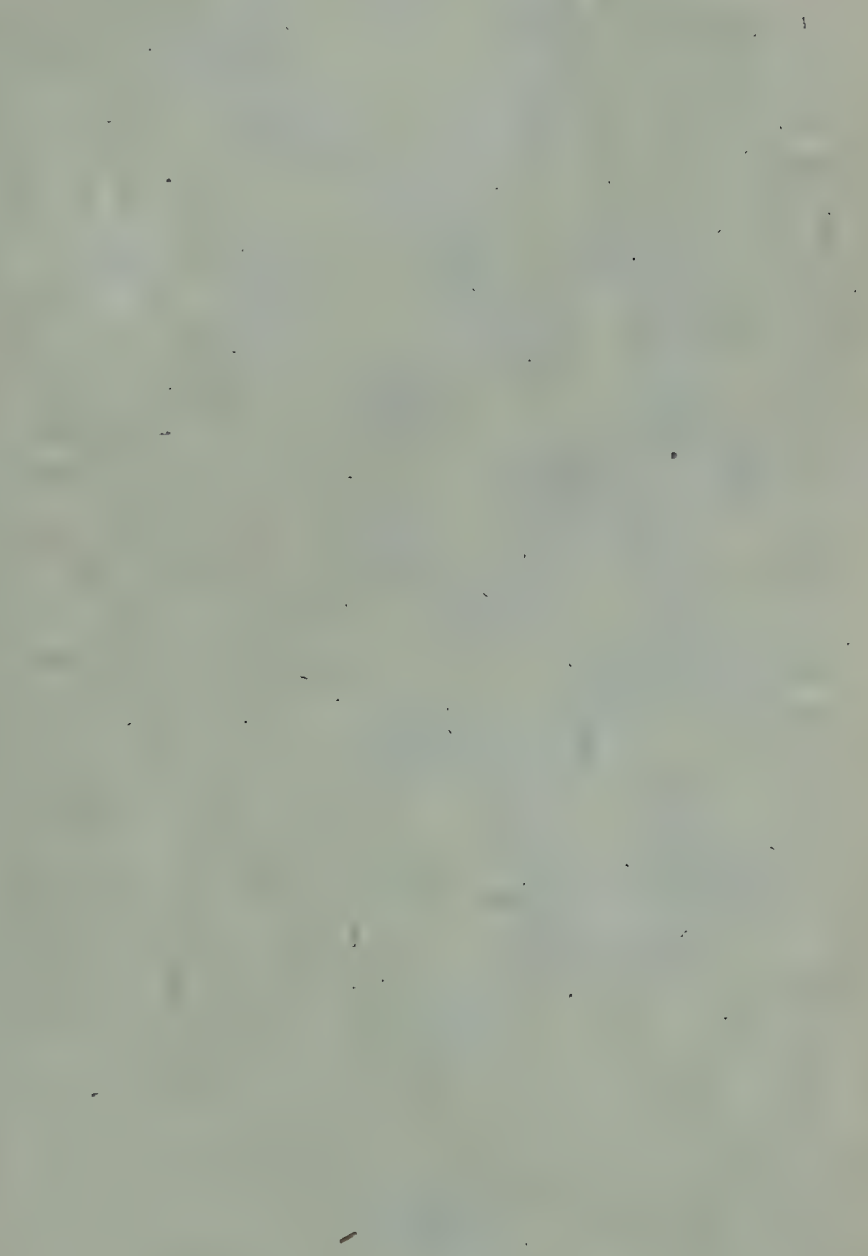

ins $\cdots=x \times x y \cdot 2$

$$
w, \ldots i
$$

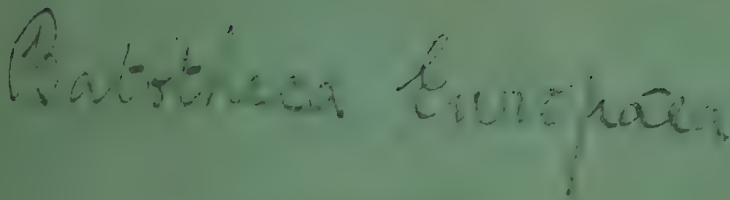

Ijecicisi $\bar{V}$

1907

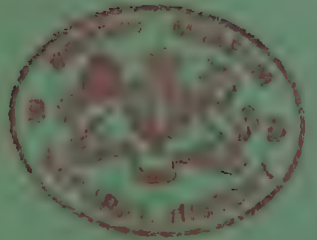





\section{BATOTHECA EUROPEA}

PUBLIE PAR

\section{H. SUDRE}

Professeur à l'Ecole normale de Toulouse (France)

12, rue André-Délieux

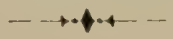

FASCICULE V - 1907

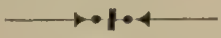

Parait par fascicules annuels de 50 numéros. Les collaborateurs qui fournissent 5 numéros convenus d'avance et préparés à 200 parts, recoivent un lascicule en échange. Chaque exemplaire doit comprenire, autant que possible, deux rameaux, l'un florifere et l'autre fructifère, plusieur's feuilles caulinaires et quelques pétales préparés à part. Les espèces déja distribuées ne sont admises que si elles diffèrent quelque peu des formes publiées.

\section{Prix du fascicule : $25 \mathrm{fr}$.}

\section{एक \\ COLLECTEURS POUR LE $5^{\circ}$ FASGICULE}

$\times \mathbf{N}$. Boulay, de Lille (Nord), nos $20 \%, 249$.

$\times$ Brochon, de Bordeaux, no 217.

Coilliot, au Mans (France), nos 216, 221 (p. p.).

Figert, professeur à Lieśnitz (Silésie), no 242.

D. W. O. Fockre, à Brême (Allemagne), nos 218, 223, 231.

Gravet, à Louette-St-Pierre (Belgique), nos 228, 229, 230.

A. Henry, au Mans (France), nos 212, 213, 216, 221, 235.

H. Hofmann, profesieur a Gloxsenhain (Saxe), nos 201. $2013,215,216$,

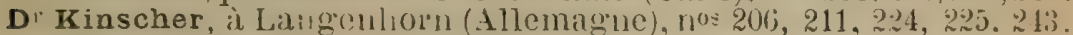

$X$ P.-J. Müller (Alsace), $n^{n s} 204.205$.

A. Ninck, ingérileur à Bar-1e-Duc (France), nos 232, 247.

$\times$ Questier (France), nos 202, 210, 214, 219, 248, 250.

Spribille, professeur a Hohensalya (Allemague), nos 208, 239, 210, 241,244 .

Sudre, professeur à Toulouse (France), noz 209, 215, 220, 29.), 2:0), $227,233,234,236,237,238$.

Ce fascicule renferme 21 Rulus qui n’ont jarnais été distrilués dans des cxsiccata numérotris; il ne contient que des R. suberecti et des $R$. silcutici. Le prochain scra consacré aux $R$. silcutici (I)iscoloroides) et aux $R$. discolores.

H. SUUDR. 
Sect. I. - Suberecti P.-J. Müll.

201 - R. SUIARTES Vest. - Saxe, où il est rare (H. Hofmann).

20: - R. GULCATUS Vest. - France; forêt de Villers-Cotterets (Questier).

(H. Hofmann).

N'est pas spontané en Europe.

204 - Te. PLIC TUS W. et N. - Alsace (Müller).

Cette forme albiflore paraît correspondre très exactement au type des Rubi germanici.

205 - R. PITCATES W. et N. s.-var. rosulentus P.-J. Müll. - Alsace (Müller).

La feuille caulinaire manquant, j'en ai reproduit une par autographie; elle a été prise vers la base du turion; celles du milieu ont les folioles un peu acuminées.

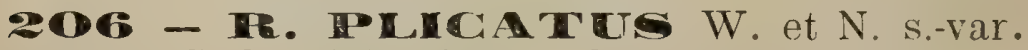
latipetalus Sud. et Kinscher. - Silésie (Kinscher).

Diffère de la sous-var. rosulentus ( $\mathrm{n}^{\circ} 205$ ) par sa denticulation plus grossière, et surtout par ses pétales plus larges, courtement ovales ou suborbiculaires, plus brusquement contractés à la base. Dans les exemplaires de $R$. rosulentus de Müller on trouve quelques fleurs à pétales relativement larges, mais ici ce caractère s'observe sur tous les rameaux.

208 - Fe. FRECATES W. et N. var. interfoliatus (N. Boul.)! - Vosges (Boulay).

Aiguillons forts ; denticulation grossière ; inflorescence feuillée ; feuilles fortement plissées, épaisses. Me paraît n'être qu'une simple variété du type.

208 - R. opacus Focke var. smiglensis (Spribille)! Allemagne : Posen (Spribille).

Ce $1^{\circ}$ diffère du $R$. opacus $F$. par ses anthères poi- 
lues et ses sépales lâchement réfléchis. Il tient du $R$. plicatus par la forme de ses folioles caulinaires terminales, et du $R$. nitidus par ses autres caractères; les bractées portent quelques glandes fines et courtes.

209 - R. NITIDUS W. et N. var. hamulosus Lef. et M. - France: Hte-Garonne (H. Sudre).

Je crois que la var. hamulosus correspond à peu près au type de l'espèce; dans le no 209 les fleurs sont un peu plus vivement colorées que d'habitude, et par ce caractère la plante rappelle un peu le $R$. holerythrus F.

210 - R. NHTIDUS W. et N. Sbsp. $R$. integribasis P.-J. Müll. - France : Valois (Questier).

Je place dans ce groupe les formes à fleurs blanches ou rosées, à étamines lungues. La foliole caulinaire terminale n'est qu'exceptionnellement entière à la base, mẻme dans les types de Müller des Ronces vosgiennes (nos 21,21 bis, 21 ter).

21-18. NITH IDU W. et N. Sbsp. Tr. integnnibasis P.-J. Müll. - Allemagne : Hambourg (Kinscher).

Ce no, qui représente le $R$. holsaticus Erichsen! est exactement le type $R$. integribasis.

212 - TR. integribasis P.-J. Müll. var. pseudosulcalus Sud. - France: Sarthe (Henry).

Turion canaliculé, foliole caulinaire terminale largement ovale, émarginée, aiguë ou brièvement acuminée, les inférieures courtement pétiolulées ; inflorescence peu armée ; sépales réfléchis.

21: - R. integribasis P.-J. Müll. var. corlitispinus Sud. - France: Sarthe (Henry).

Diffère du K. inlegribasis par ses aiguillons beaucoul' plus forts, son inflorescence très armée, ses pétales grands et largement ovales, blancs, ses étamines très longues. Ses feuilles raméales supérieures sont parfois subdiscolores, ce qui m'avait porté tout d'abord à rattacher la plante au R. affinis W. et N. Elle tient du $R$. integribasis par ses fleurs blanches, et du $R$. holerythrus par ses grands aiguillons. Croit aussi en VIaine-et-Loire et dans la Loire-Inférieure. 
21 1 - Re. integnibasis P.-J. Müll. Microg. R. nitidiformis Sud! - France : forêt de Retz (Questier).

Remarquable par ses grands aiguillons, ses calices aculéolés, étalés, et la pilosité de la face inférieure des feuilles, qui rappelle celle du $R$. pyramidalis Kalt. Questier l'appelait $R$. appendiculatus (non Tratt.) et Godron le lui avait déterminé $R$. affinis. Il est intermédiaire entre $R$. nitidus et $R$. carpinifolius; par ses sépales nettement verts et bordés de blanc, il appartient aux $R$. suberecti. An $R$. nitidus $\times$ pyramidalis? Cela me paraît très douteux.

M. Spribille a récolté à Gross-Strehlitz (Silésie) une forme qui me paraît être identique au no 214.

P 5 - F . indutus Boul. et Vendr. - France : Tarn (H. Sudre).

Intermédiaire sous certains rapports entre $R$. nitidus W. N. et $R$. affinis W. N. et paraissant se placer à la suite du R. integribasis P.-J. Mïll. Il existe en effet des formes assez nettement intermédiaires entre $R$. integribasis et $R$. indutus, en particulier la plante distribuée dans Set of British Rubi, $n^{\circ} 106$, sous le nom de $R$. opacus fa minor Focke (R. subopacus sud.) et une autre forme trouvée en Maine-et-Loire par M. Bouvet. S'éloigne sensiblement du R. affinis W. N. par la forme de ses folioles et son inflorescence. N'est pas rare dans le Tarn, d'ou je la possède de plusieurs localités.

21 - He. holemyhnus Focke. - France: Sarthe (Henry et Coilliot).

Cette espèce se présente sous des formes faibles, à foliole caulinaire terminale peu échancrée à la base (Bat. eur. n० 52), correspondant au R. burdigalensis Boul. et Clavaud, Assoc. $* u b .599,668,670$, et rappelant le $R$. nitidus W. et $\mathrm{N}$.; mais dans les formes vigoureuses, comme dans ce no 216 et le suivant, elle rappelle souvent d'une facon frappante, par la forme deses feuilles dont la foliole caulinaire terminale est profondément cordée et acuminée, le type $R$. affinis, représenté par le $n^{\circ} 21 \%$; les pédoncules sont toutefois plus étalés et les organes tloraux vivement colorés. Sur certains spécimens d'herbier la distinction des $R$. holerylhrus et affunis est difficile a faire, surtout si la couleur de la fleur n'est pas notée. 
217 - Th. holerythrus F. - France: Gironde (Brochon).

Ce numéro, que j'ai reçu de M. le $\mathrm{D}^{\mathrm{r}}$ Bouly de Lesdain, était destiné aux Rubi Gallici. La plante a dủ pousser sur un terrain gras; certaines feuilles caulinaires, à foliole terminale très profondément cordée, paraissent identiques à celles du $R$. vigorosus M. et Wirgt, qui par ses fleurs blanches, appartient au R. affinis W. et $\mathrm{N}$.

Obs. - C'est par erreur que, sur les étiquettes des nos $215,216,21 \%$, le nom spécifique a été imprimé en CAPITARES VORMANDE; il aurait dû l'être en petites normandes.

218 - R. ARING W. et N: - Allemagne (Focke).

Sect. II. - Silvatici P.-J. Müll.

A -- Grati Sud.

$$
\text { a - Eugrati. }
$$

219 - R. CATPINTHLTU Wh. Microg. Re. platybelus Sud. - France : Oise (Questier).

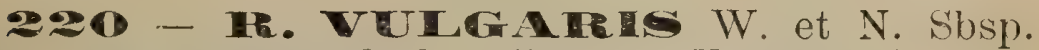
IR. Closianus Sud. - France : Tarn (H. Sudre).

Robuste; turion glauque, à aiguillons forts et denses; feuilles vertes ou un peu grises-tomenteuses en dessous, à dents fines, irréguliêres ; foliole caul. terminale obovale, entière, brusquement acuminée; rameau à aiguilons forts, falqués. Inflorescence grande, feuillée et interrompue à la base, multiflore, à aiguillons nombreux, déclinés ou falqués; calice poilu, un peu aculéolé, imparfaitement réftéchi ; 1leurs rosées; carpelles velus ; fertile. Croît dans le voisinage du $R$. fagicola de Martr. - Dédié à M. le Dr Clos, Directeur honoraire du Jardin des Plantes de Toulouse, ancien professeur de botanique à la faculté des sciences, et originaire de Sorèze, dont il a longtemps étudié la flore.

221-T.CLETEROPHILUS Gen. France : Sarthe (Henry et Coilliot). 
222 - X R. TENUICUSPIS Sud.; $R$. clethrophilus $\times$ tomentosus (Lloydianus). - France : Tarn (H. Sudre).

Croît dans le voisinage àu $R$. clethrophilus, qu'il rappelle par la forme de ses folioles, mais est discolore comme le $R$. tomentosus et a l'inflorescence poiluehérissée et garnie de petits aiguillons jaunâtres. Stérile.

\section{2ะ3 - TR, ARATUS F. - Allemagne (Focke). !}

224 - R. GTR TUSF. Sbsp. TR. sciocharis Sud. - Allemagne: Holstein (H. Kinscher).

Comme il existe un R. sciophilus Lef. et M. bien antérieur à celui de J. Lange, j'ai dû changer le nom de cette sous-espèce, qui se rapproche un peu du $R$. danicus par ses anthères poilues.

225 - HE. DANICUS Focke. - Allemagne: Holstein (Kinscher).

Par ses sépales nettement étalés, son inflorescence plus armée, ses anthères poilues, cette espèce me paraît bien distincte du $R$. macrophyllus W. et $\mathrm{N}$.

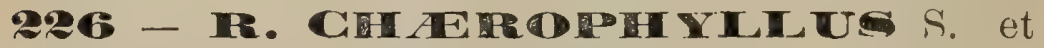
Sch. Microg. R. brachythyrsuides Sud. - France : Corrèze (H. Sudre).

Cultivée de graines prises sur les échantillons que j'ai récoltés à Bort. La runce est âgée de ô ans. Elle a tous les caractères de la plante sauvage, toutefois l'inflorescence est moins glanduleuse et les feuilles sont encore tout à fait virescentes, au lieu d'être un peu grises-tomenteuses en dessous; le calice reste manifestement étalé, et ce caractère me paraît de premier ordre. Ce $n^{\circ}$, fourni par un buisson encore très jeune, sera complété plus tard.

22y - Th. brachythyrsus Sud. - France: Luchon (H. Sudre).

Se place dans le voisinage du $R$. chcerophyllus Sag. et Sch. Spécial aux Pyrénées centrales. 


\section{b - sprengeliani.}

228 - $\times$ R. ORTHOCLADOIDES Sud. $R$. orthocladus $\times$ pyramidalis. - Belgique (F. Gravet).

Faciès du $R$. orthocladus A. Ley $\left(B a l . \mathrm{n}^{0} 56\right)$, mais turions plus anguleux, pilosité des feuilles et des axes florifères rappelant le $R$. pyramidalis Kalt.; pollen très imparfait ; plante peu fertile.

229 - R. ORTHOCLANUA A. Ley, Microg. R. euchlous Focke. - Belgique (F. Gravet).

Turion couvert de nombreuses glandes pâles, inégales; stipules glanduleuses, ainsi que les pétioles; folioles plus longuement acuminées que dans le $R$. orthocladus, de forme plus allongée, moins arrondies à leur base. M. Focke (ap). Asch. et (ìr. Syn. VI $p .470$ ) appelle $R$. cuchlous le type $R$. orthocladus A. Ley, tandis que dans l'herbier de M. Gravet il applique ce nom à la forme distribuée. Tout en reconnaissant que les nos 56 et $2: y$ sont très affines, jc ne crois pas qu'il soit possible de les réunir. A ce sujet, voici ce que m'écrit M. Gravet: "Je crois que le R. orthocladus A. Ley est bien distinct dı $R$. euchlous F. Du reste M. Focke me les a toujours nommés séparément ".

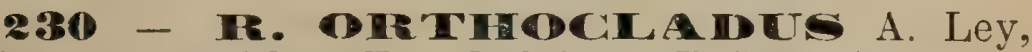
Microg, R. euchlous F. - Belgique (F. Gravet).

C'est la plante sauvage. Ses caractères se sont bien conservés par la culture et sont plus apparents sur le $\mathrm{n}^{\circ}$ précédent. Il est possible que le $R$. euchlous dérive du $R$. orlhocladus par croisement, c'est pour cela que je le place à còté de ce dernier ; par son hétéracanthie, il se rapproche du $R$. infestus Wh., de la série des $R$. Radule.

231 - R. A RTRHE ENII Lange. - Allemagne: Brême (Focke).

23:. - Me. Ninckii Sud. - France : Marne (A. Ninck).

Turion à faces planes, à quelques poils épars, subglaucescent, à glandes sessiles; feuilles presque toutes 3-nées, amples, tlasques, à poils épars en dessus, vertes et à poils brillants en dessous, assez grossièrement dentées; foliole caul. terminale ovale, échancrée, acu- 
minée. Rameau làchement poilu, à aiguillons déclinés ; inflorescence courte, un peu feuillée, poilue-herissée, à glandes fréquentes, mais la plupart subsessiles, à aiguillons faibles, déclinés ; sépales tomenteux, hérissés, aculéolés, lâchement relevés sur le fruit ; pétales obovales, d'un beau rose vif ; étamines pourpres, dépassant peu les styles verdâtres; carpelles glabres; pollen peu mélangé. Plante intermédiaire entre les $R$. gratus F. et Sprengelii Wh. M. Ninck a observé de nouveau cette ronce en 1907 ; ce n'est qu'exceptionnellement qu'elle présente quelques feuilles à ‘े folioles. Abonde sur plusieurs points du voisinage.

233 - TE. HTCUSO Sud. - (spi. collecl.) Microg. R. teretipes Sud. - France : Tarn (H. Sudre).

Les caractères communs aux formes de ce groupe sont: Turion obtusément anguleux ou arrondi, glabre ou peu velu, à aiguillons égaux ou presque égaux, à glandes nulles ou rares; feuilles 3-5-nées, la plupart grises ou blanches-tomenteuses en dessous; inflorescence ordinairement glanduleuse ; étamines plus courtes que les styles ou les égalant; calice élalé ou relevé sur le fruit; réceptacle velu.

Le $R$. teretipes a le turion glabre et glaucescent, les feuilles finement et superficiellement dentées, la foliole caul. terminale ordinairemeut obovale, assez brusquement acuminée ; l'inflorescence très courtement poilue, un peu glanduleuse, à aiguillons faibles; le calice tomenteux, à lobes appendiculés, lâchement relevés sur le fruit, et les fleurs roses, à étamines très courtes. La forme distribuée (var. pilocarpus) a ]es carpelles velus et les fleurs beaucoup plus pâles que le type, qui n'est pas rare dans le Tarn. Ces formes, rappelant le $R$. Sprengelii Wh. par leurs étamines courtes, en sont toutefois bien distinctes par leur feuillage discolore. - Le $n^{0} 233$ a été cueilli dans un endroit frais et couvert.

2\$ - R. saltuivagus Sud. - France : Pyrénées (H. Sudre).

Plus hétéracanthe que les autres formes de ce groupe; se rapproche des $R$. vestiti. Très fertile. 
B - Euvirescentes Gen.

a-Calvescentes Gen.

235 - X GALISSIERI Sud. ; R. Questieri $X$ ulmifolius. - France : Sarthe (Henry).

Faciès du R. Questieri, mais turion glaucescent, feuilles un peu discolores, axe tlorifère peu poilu, étamines moins longues; plante stérile.

236 - M. QUESTERE Lef, et M. var. Acutifolius Sud. - France : Ariège (H. Sudre).

Aiguillons un peu coniques; foliole caulinaire terminale largè, ovale ou obovale, aiguë ou cuspidée; pétales aigus, entiers; styles ordinairement verdâtres. C. aux environs de Foix ; croît avec les $R$. clethrophilus Gen. et elongatispinus Sud. Fertile.

238 - TE. FATCOLA de Martr. - Tarn: (H. Sudre).

Me parait bien distinct du R. Questieri et je ne crois pas qu'on puisse l'y subordonner. Ne descend guère au dessous de $300 \mathrm{~m}$.

238 - MAdUCUCUS Sud. - France: Tarn (H. Sudre).

D'abord rapproché du R. macropluyllus par l'ablié Boulay, mais plus tard reconnu bien distinct ; est intermédiaire entre ce dernier et R. Queslieri. AC. dans tout le département du Tarn. Reconnaissable à ses turions plans, un peu pubescents ; a ses folioles terminales obovées, cuspidées; à son inflorescence peu poilue, églanduleuse, à pédoncules ascendants; à ses fleurs blanches, à pétales étroits, distants.

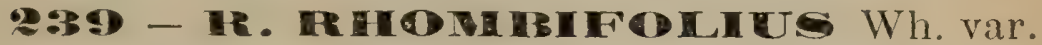
pyramidatiformis spribille Ver. Bot. ler. Prov. Brand. (1907) p. 1399 ; R. negleclus ej. (prius) non P.-J. Müll. - Allemagne: Silésie (Spribille).

Aiguillons forts sur le raneau et sur l'intlorescence; styles pailes. Pour le reste semblable au type.

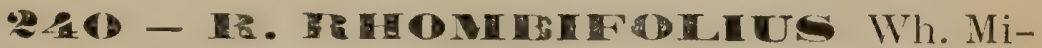
crog. R. Wimmerianus siprib.! - Allemanne : silésie (Spribille).

Diffère du R. rhombifolius par ses turions glabres- 
cents, son inflorescence très feuillée, ses fleurs blanches et ses étarnines courtes.

2R1 - SULESIACUS Wh. - Allemagne: Silésie (Spribille).

2\% - H. STESIACS Wh. var. tabanimontanus (Figert)! - Allemagne: Silésie (Figert).

Turion glabre, à aiguillons subégaux; feuilles glabrescentes en dessous ; denticulation grossière ; axe florifère lâchement poilu, un peu glanduleux; étamines dépassant les styles: tels sont les caractères qui distinguent cette plante du type $R$. silesiacus.

243 - TR. GILESRACS Wh. Microg. R. silingicus Kinsch. ! - Allemagne: Silésie (Kinscher).

Bien distinct du $R$. silesiacus Wh. par ses folioles caul. terminales entières à la base et courtement pétiolulées, ses glandes bien plus fréquentes et ses étamines plus courtes que les styles. Est voisin du $R$. vallicolus Müil., mais non identique.

24 - Te. GILESIACU Wh. Microg. R. amygdalanthoides Sprib. 1 - Allemagne: Silésie (Spribille).

Caractères du $R$. silesiacus, mais pétales et étamines d'un rose vif. Intermédiaire entre cette espèce et $R$. amygdalanthus $\mathbf{F}$, que je considère comme une sous-espèce du $R$. silesiacus Wh. M. Spribille a décrit cette plante en 1907 (Ver $\%$. Bot. Ter. Prov. Brandy. IL, p. 191).

b - Piletosi Gen.

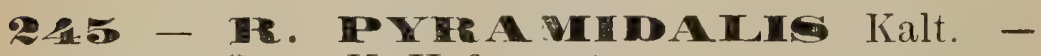
Allemagne: Saxe (H. Hofmann).

246 - M. MACIEOPHYLUG W. et N. - Allemagne : Saxe (Hofmann).

J'avais jusqu'à ces derniers temps distingué le $R$. piletostachys God. du type $R$. macrophyllus W. et N., ainsi que l'avaient fait M. Focke (Syn. Rub. Germ.) et l'abbé Boulay ( $a p$. R. et C. $F l$. Fr. VI) et en me basant sur les spécimens de $R$. macrophyllus distribués par Wirtgen (Hb. rub.rh.éd. $\left.1 \mathrm{n}^{0 \mathrm{~s}} 79,80, e ́ d .2 \mathrm{n}^{0 \mathrm{~s}} 16 \mathrm{et} 17\right)$. Bien que ces sfécimens correspondent à la description des Rubigermanici, je crois qu'ils représentent 
une forme aprique de l'espèce: les aiguillons du rameau sont en effet plus forts que d'habitude, et la plante est un peu discolore. Cette forme, que Müller avait appelée $R$. megaphyllus in Flora (1858). $Z$. $u . B$; Pollich. (1859), '293] et que M. Kretzer avait distribuée dans l'Herb. eur. de M. Baenitz sous le nom de $R$. W'interi f. latifolia, ne m'est connue que de Westphalie, des provinces rhénanes et de Lorraine ; elle est beaucoup plus rare que celle que l'on désigne habituellement sous les noms de $R$. macrophyllus ou de $R$. piletoslachys, et qui est largement répandue dans toute l'Europe centrale. Aussi j'estime que c'est cette dernière qui doit être envisagée comme le type de l'espèce. La var. megaphyllus mise à part, je ne vois pas qu'il soit possible de conserver une var. piletostachys du $R$. macrophyllus. Pour montrer combien la distinction est difficile à faire, il me suffira de rappeler: $1^{\circ}$ que le numéro 8 bis des Ronces vosyiennes distribué sous le nom de $R$. piletoslachys par N. Boulay est rattaché au type par ce batologue dans la Flore de Rouy et Camus; ;0 que le $n^{n} 1660$ des exsiccata de Billot, rapporté au type $R$. macrophyllus par M. Focke, est très exactement un $R$. piletostachys God. ! et a été déterminé comme tel par Godtron lui-même (in $h b$. Questier) $13^{\circ}$ enfin que la plante de Brême publiée par M. Focke dans l'Association rubologique ( $\left.\mathrm{n}^{\circ} 603\right)$ sous le nom de $R$. macrophyllus et que M. Focke et l'abbé Boulay rattachent au type de l'espèce, ne diffère en rien de la plante de Godron. - En réalité, dans une même centurie, ainsi que je l'ai remarqué sur les $\mathrm{n}^{0 \mathrm{~s}} 246,247,248$ du Balotheca, on rencontre des rameaux dont l'inflorescence est maigrement hérissée et qui, pris isolément, se rattacheraient au type, et d'autres, plus trapus, à axe florifère épais et fortement hérissé, qui seraient des $R$. pilelostachys très typiques. Ceci nous montre que, dans ce genre particulièrement litigieux, les espèces ne peuvent être sû'cment interprétées que lorsqu'elles sont représentées jar de bons et abondants matériaux. Aussi n'aije pas hésité à distribuer 3 numéros consécutifs qui représenteront largement le type $R$. macrophyllus tel que je le comprends.

Le no 246, que M. Hofmann arait d'abord appelé R. danicus (non F.), me paraît représenter exactement la plante que IVeihe avait nommée $R$. Wimmeri ( $a p$. Wimm, et Grab. Fl. sil. II $p$. 2). Ce R. Wimmeri, d'après les recherches faites par mon dévoué collabora- 
teur M. Kinscher, et les spécimens de $R$. macrophyllus de Silésie qu'il a bien voulu m'adresser, n'est pas autre chose qu'un $R$. piletostachys; il est représenté dans l'Herb. eur. de M. Baenitz par le no 9056 (R. macrophylloides Utsch, non Gen.) récolté dans le voisinage de l'une des trois localités classiques du R. Wimmeri (M. Kinscher).

248 - R. MACROMIIYLCUS W. et N. - France : Meuse (Ninck).

Ce $n^{0}$ représente un $R$. pileloslachys. La plante est très vigoureuse et a dù pousser sur un terrain fertile. Les fleurs sont ordinairement rosées, mais parfois tout à fait blanches, comme dans la plante des Kubi germanici.

248 - RE. MACIROPHTLIUUSW. et N. - France : Valois (Questier).

C'est la plante distribuée par Billot sous le $n^{0} 1660$; Godron l'avait appelée R. piletostachys!

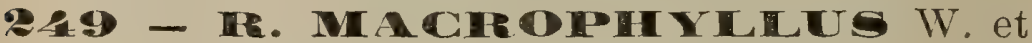
N. var. Boulayi Sud. -- France (N. Boulay).

Ce $n^{\circ}$ avait été préparé pour les Rubi gallici. Après la mort de l'abbé Boulay, il me fut donné par son collaborateur M. le $\mathrm{D}^{\mathrm{r}}$ Bouly de Lesdain, mais sans autre étiquette qu'un bout de papier portant le mot " $R$. macrophyllus ". Il est remarquable par ses feuilles très fineinent dentées et ses folioles terminales nettement obovales, ordinairement entières à la base, cuspidées au sommet; par ce caractère il se rapproche du $R$. Schlechlendalii Wh. Toutefois ses turions nettement anguleux, très vigoureux, me le font placer à côté du $R$. macrophyllus.

Il provient apparemment du nord de la France, mais il m'est impossible de préciser davantage.

250- TR. GCHICIECHTENDACI Wh.

- France: Oise (Questier).

Turion plus faible et plus velu que dans le $R$. macrophyllus ; aiguillons moins forts ; foliole terminale obovée. C'est le R. Schlechtendalii de Müller (Tersüch).

\section{H. SUDRE.}

Novembre $190 \%$.

Albi.-Impr. A. Nouguiês, rue de l'Hôtel-de-Ville, 33 
(n) 

Cusce $150 \cdot \times \times \times V=$

$\frac{2.2 n d i c}{2.2}$

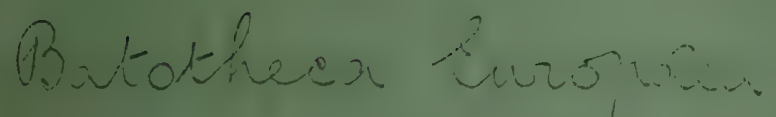

fascione VI

$$
1908
$$





\title{
BATOTHECA EUROP在A
}

PUBLIÉ PAR

\begin{abstract}
H. SUDRE
Professeur à l'Ecole normale de Toulouse (France)

12. rue André Délieux

FASCICULE VI - 1908

$\longrightarrow$.

Paraît par fascicules annuels de 5ొ0 numéros. Tes collaborateurs qui fournissent 5 numéros convenus d'avance et préparés à $20 \%$ parts, regoivent un fascicule en échange. Lihaque exemplaire doit comprenilre, autant que possible, deux rameaux, l'un tlorifere et l'autre fructifère, plusieurs feuilles caulinaires et quelques pétales préparés à part. Les espèces déja distribuées ne sont admises que si elles diffèrent
\end{abstract} quelque peu des formes publiées.

\section{Prix du fascicule : 25 ir.}

\section{resorem \\ COLLECTEURS POUR LE $6^{\circ}$ FASGICULE}

Dr Bouly de Lesdain, à Dunkerque (Nord), no 281.

Coilliot, au Mans (Sarthe), nos 283, 294.

A. Henry, au Mans (Sarthe), n’s 263, 273, 282, 295.

E. Holzfuss, prof. à Stettin (Poméranie), nos 251.255.

D. H. Kinscher, à Péterwit\% (Silésie), nos 257, 261, 270.

A. Ley, à Ross (Angleterre), nos 252, 262.

$\times$ Questier, Thury-en-Valois (France), nos 266, 268.

Dr $\mathbf{H}$. Sabransky, â Soechau (Styrie), no 592.

Spribille, prof à Hohensalza (Posen), no3 253, 265.

H. Sudre, yrof à Toulouse, nos 254, 256, 258-60, 264, 267, 269, 271-2, 274-280, 284-9, 291, 293, 296-300.

Zinsmeister, Wengen, Bürgheim (Bavière), no 290.

\section{OBSERVATIONS}

Je prie les collaborateurs de vouloir bien m'adresser leurs plantes oers la fin de septembre, la préparation du batotheca ayaut lieu au commencement d'octobre.

Le fäscicule VII sera consacré aux $R$. discolores P.-J. M., mais ou peut préparer des Rubus appartenant à d'autres sections, pourvu qu'uls n'xient pas été distribués.

Ce fascicule renferme 36 Rulous qui n'ont jamais été publiés dans des exsiccata numerrotés.

H. SUURE. 
Sect. I. - Suberecti P.-J. Müll.

251 - P. FISSUS Lindl. - Poméranie (E. Holzfuss).

25: - R. FISSUS Lindl. - Angleterre (A. Ley).

La plupart des spécimens proviennent du comté de Brecon; quelques feuilles ont été récoltées dans celui de Gloucester.

\section{Sect. II. - Silvatici P.-J. Müll.}

Gr. a - Grati Sud.

\section{3 - F. VUCGATE W. N. - Silésie (Spribille).}

M. Spribille m'a adressé cette plante sous le nom de $R$. rhamnifolioides Sprib. (ad. int.) Je crois que ce n'est qu'une variation aprique du type vulgaris. Dans la plupart des rameaux florifères le calice est lâchement réfléchi, mais c'est surtout peu de temps après l'anthèse que la direction des sépales est importante à noter.

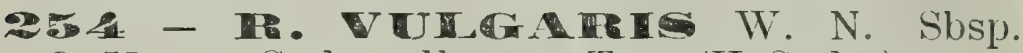
T. fallens Sud. - France, Tarn (H. Sudre).

Diffère du $R$. vulgaris W. N. par ses turions subarrondis. ses aiguillons moins denses, ses feuilles presque simplement dentées, à folioles caulinaires terminales plus nettement obovées, son inflorescence peu armée, à pédoncules plus ascendants. Plante peu ou point glanduleuse, à pollen très peu mélangé.

255- Re. Ge AUE F. v. Holzfussii Sud. Poméranie (E. Holzfuss).

Diffèredu $R$.gratus F., auquel il respemble beaucoup, par ses turions lâchement poilus, à faces planes, son inflorescence moins feuillée et son pollen fortement mélangé. Peut-être dérive-t-il du $R$. gratus par croisement?

256-R. subglaucus Sud. - Tarn (H. Sudre).

J'ai récolté cette plante un peu trop tard; le calice, 
qui est d'abord étalé, se renverse à la fin. I a place de ce Rubus reste douteuse. Il rappelle un peu le $R$. vallisparsus Sud., de la série des $R$. rules, mais est moins hétéracanthe et a le turion plus anguleux. 'Turion très glauque.

25 - $\mathbf{z}$. ORTHOCLADUS A. Ley v. transsudeticus Kinscher! - silésie (Kinscher).

Diffère du type par sa dentelure moins vive, ses feuilles caulinaires J-nées, à folioles terminales largement ovales, cordées, par son inflorescence bien plus armée et ses sépales aculéolés.

258 - R. FICTUS Sud. Microg. R. albulus Sud. Tarn (H. Sudre).

Cette plante étant décrite dans le 1 er fasc. de mes Rubi Europe je me borne à dire qu'elle est caractérisée par ses turions épruineux, courtement poilus, ses feuilles tris discolores, finement denticulées, les caul. pédato-quinées, ses axes brièvement poilus ; elle est églanduleuse.

25\$-R. FICTUS Sud. Microg. R. teretiramus Sud. - Tarn (H. Sudre).

Pour la description, voir mes Rubi Europe. Le calice reste longtemps étalé, mais finit par se réfléchir à la maturité du fruit. Les circonstances ne m’ont pas permis de récolter plus tôt cette plante. Je compléterai ce $\mathrm{n}^{\circ}$ quand il me sera possible de cueillir des rameaux florifères.

260 - R. teretipes Sud. var. validus. - Tarn (H. Sudre).

Robuste ; feuilles caul. 5-nées, à folioles terminales ovales, carpelles poilus. Turion glaucescent.

261 - R. Schwenckfeldii Kinscher. - Silésie (H. Kinscher).

Bien que les sépales soient réfléchis sur les rameaux fructifères, ont voit qu'ils sont étalés après l'anthèse. Par ses étamines courtes et ses sépales verdâtres et bordés de blanc, la plante me parait se placer dans le voisinage du $R$. cimbricus $\mathrm{H}$., dont elle diffère surtout 
par la dentelure de ses feuilles et la forme de ses folioles terminales.

$$
\text { Gr. b-Euvirescentes Gen. }
$$

$26:-$ R. MAASSII F. Sbsp. R. oxyanchus Sud. var. silurum (A. Ley!) - Angleterre (A. Ley).

Etudiée sur de nombreux sjécimens la plante se montre normalement virescente et s'éloigne sensiblement du R. rhamnifolius W. N. Le $\mathrm{n}^{0} 109$ de Sel of British Rubi, qni est moins typique que les échantillons du Balotheca, a dû être cueilli dans un endroit découvert, car il est un peu discolore.

$263-$ R. MAASSII F. Microg. R. viridicatus Sud. - Maine-et-Loire (Henry).

Identique à la plante d'Ifort Bridge (S. Hants).

Les fruitssont tombés sur quelques rameaux ; la plante est fertile.

262 - R. opentus Sud. - Hte-Garonne (H. Sudre).

Un peu plus grêle que le type des Pyrénées, folioles moins larges, axe tlorifère moins poilu-hérissé, inflorescence plus lâche, feuillage un pell discolore, caractères secondaires dus sans doute à la station de la plante.

265 - F. amydalanthus Fock. var. gliviciensis Spribille - Silésie (Spribille).

Intermédiaire entre le $R$. silesiacus Wh. et le $R$. amygdalanthus F. S'éloigne de ce dernier par ses aiguillons rameaux allongés, déclinés, son inflorescence courte, large, peu poilue, plus manifestement glanduleuse. Ses tleurs roses et ses aiguillons caulinaires plus forts le distinguent du $R$. silesiacus.

$26-X$ R. PSEUDO-SCHLECHTENDALII SUd.; R. Schlechtcndaliix Sprengelii. - Valois (Questier).

J'ai extrait cotte plante de la centurie de $R$. Schlechtendalii de Questier (I3at. eur. no 250 ). Elle diffère du R. Schlechtendalii par ses feuilles caul. en partie 
3-nées, son inflorescence presque aphylle, à pédoncules étalés, ses glandes très courtes et son pollen très imparfait.

268 - R. debilispings Sud. - Tarn $(H$. Sudre),

268 - R. NEMORENSIS L ef. et M. ! - Aisne (Questier).

Espèce rare et jusqu'ici peu connue. M. Spribille m'a adressé de Silésie, sous le nom de $R$. dombrovicus Sprib., une forme qui n’est pas très éloignée du type et qui rappelle mon $R$. debilispinus par son feuillage.

\section{Gr. c - Discoloroides Gen.}

$a-$ Subvirescentes Sud.

\$69-R. carmauxensis Sud. - Tarn (H. Sudre).

Plante tenant à la fois des $R$. macrophyllus W. N., argenteus $\mathrm{W}$. et $\mathrm{N}$. et villicanlis Koehl. Diffère de ce dernier par ses turions plus velus, ses aiguillons moins forts, ses feuilles presque simplement dentées, mollement pubescentes en dessous, son inflorescence peu armée, peu ou point glanduleuse. Les sépales ne se réfléchissent pas nettement, ce qui fait que la place à donner à ce Rubus reste un peu douteuse.

280 - R. Langei G. Jensen var. parvifolius G.Jens. - Holstein (H. Kinscher).

Folioles petites, la caulinaire terminale plus étroite que dans le type, plus ou moins cunéiforme à la base. Anthères poilues.

\section{$b$ - Subdiscolores Sud.}

28 - T. A TEGENTEUS W. N. var. longicuspidatus. - Tarn (H. Sudre).

Folioles plus étroites que dans le type, longuement acuminées.

27 2 - IR. ATEGINTEUS W. N. var. castrolinensis. 
Plante âgée de 6 ans et tout à fait identique à la plante sauvage, qui croît sur le grès armoricain ; est normale depuis quelques années.

28:3 - FE. A HaGERTEUS W. N. var. armatissimus Sud. - Loire-Inférieure (Henry).

Turion velu; aiguillons très forts et très denses sur les turions, les pétioles, les rameaux et les pédoncules; feuilles très pubescentes en dessous, doublement et très inégalement dentées ; foliole caul. terminale rhombée. Plante églanduleuse ; se rapproche beaucoup de la var. ferox du R. consobrinus sud. De l'Isle avait cueilli cette plante à la Haye (Loire-Inférieure) et d'après M. Henry cette var. serait commune dans ce département.

$28-R$. aurensis Sud. - Luchon (H. Sudre).

Se rapproche du $R$. argenteus W. N. mais fructifie très mal. Dérive peut-être du $R$. consobrinus par croisement?

275 - R. consobrinus Sud. - Ariège (H. Sudre).

C'est le type de cette sous-espèce; toutefois la foliole caul. terminale est moins large que d'habitude, ce qui rapproche la plante du $R$. argenteus W.N.

$2 \boldsymbol{8} \mathbf{6}$ - R. incarnatus Müll. var. conformis sud. - Tarn-et-Garonne (H. Sudre).

Forme très vigoureuse, montrant bien les caractères de cette variété.

2ซร - Te. incanonatus P.-J. Müll. var. callichroanthus Sud. - Hte-Garonne (H. Sudre).

Plante très vigoureuse, que j'avais. d'abord placée à tort dans les $R$. discolores.

288 - Re. inca anatus P.-J. Müll. var. pseudonemoralis Sud. - Hte-Garonne (H. Sudre).

Je l'avais d'abord rapproché du $R$. villicaulis Knhl., mais après l'avoir étudié sur place j'estime qu'il est plus voisin du $R$. incarnatus Müll. 
289- Ts. enyptadenes Sud. var. pubiranus. - Tarn (H. Sudre).

Turion canaliculé, feuilles discolores, foliole terminale obovale, cuspidée, inflorescence allongée, lâche, feuillée; sépales lâchement réfléchis.

280 - TR. captadenes Sud. var. stereacanthoides. - Toulouse (H. Sudre).

Plante née en 1902 et aujourd'hui très vigoureuse, ayant tous les caractères de la forme sauvage de Bretagne; toutefois encore peu discolore.

Ce $n^{0}$ sera complété ultérieurement.

281 - Te. cryptadenes Sud. var. bipartitus (B. et Bouv.) - Seine (Dr Bouly de Lesdain).

Cette var. est commune aux environs de Paris ainsi que dans l'Anjou et la Sarthe; il est parfois difficile de la distinguer du type, qui n'est pas rare dans le N.-O. de la France.

\section{2 - R. oplothyrsus Sud. - Sarthe (A. Henry).}

Turion glaucescent, inflorescence très armée, à bractées un peu glanduleuses. liot).

283 - R. oplothyrsus Sud. - Sarthe (Coil-

284 - Pe. multivagus Sud. - Tarn $(H$. Sudre).

Turion obtus, glabrescent; feuilles 3-4-5-nées, mollement pubescentes et grises ou blanches-tomenteuses en dessous ; foliole terminale largement ovale ou suborbiculaire, un peu émarginée à la base, brièvement acuminée; inflorescence courte, peu feuillée et peu armée, poilue, munie de bractées incurvées et de glandes subsessiles. - Rappelle le $R$. bifrons West mais a le turion plus obtus, les feuilles moins discolores, velues en dessous, les caulinaires très souvent 3-nées. Commun dans la région montagneuse du Tarn. 


\section{5 - T. gneissogenes Sud. - Tarn $(H$. Sudre).}

Le calice n'est pas nettement réfléchi, et lorsque la plante croît à l'ombre, elle ressemble beaucoup à certaines variétés de $R$. carpinifolius Wh.qui croissent dans les mêmes lieux. A aussi un peu les apparences du $R$. pyramidalis Kalt., mais est plus discolore.

28 - R. LASIOTHYRSUS Sud. var. flavescentispinus. - Ariège (H. Sudre).

$98 \boldsymbol{8}-\times$ R. INOPS Sud. - Ariège (H. Sudre).

J'avais cru que cette plante était un $R$. continens $X$ ulmifolius; après l'avoir observée de nouveau sur place je crois qu'elle dérive plutôt du $R$. lasiolhyrsus, mais l'autre parent reste problématique. Stérile.

288 - R. ALTERNIFLORUS M. et Lef. var. gymnostachys. - France (H. Sudre).

Cette plante est née en $190^{2}$; elle ne diffère en rien de la for'me sauvage. Peut-être est-ce un hybride fertile des R. adscitus Gen. et ulmifolius Schott?

289-R. oreigenus Sud. - Puy-de-Dôme (H. Sudre).

290 - IR. sueviacus Sud. - Souabe (Zinsmeister).

Se distingue des autres sous-espèces du $R$. alterniflorus M. et L. par ses turions non pruineux, glabrescents; ses feuilles mollement relues-veloutées en dessous, à voils pectinés le long des nervures; ses folioles caul. terminales largement ovales ou un peu obovées, brièvement acuminées; par son inflorescence feuillée, lâchement poilue, munie de glandes éparses et d'aiguillons nombreux, allongés, la plupart presque droits. Carpelles velus. Rappelle le $R$. villicaulis Kohl. par ses grands aiguillons et le $R$. pyramidalis Kalt. par la pilosité de ses feuilles.

291 - Tr. soparinus Gen. - Vendée (H. Sudre). 
292 - TR. durimontanus Sabrs. - Styrie. (Sabransky).

Cette plante a le pollen peu mélangé et me paraît bien fructifier; je ne la crois pas hybride; elle est un peu glanduleuse vers la base de l'inflorescence, ce qui me la fait ranger dans le groupe du $R$. alterniflorus M. et I. Elle est surtout caractérisée par ses turions épruineux, velus, sa dentelure très aigüe et irrégulière, son inflorescence très brièvement foilue, peu armée et très peu glanduleuse.

29:B - He. obvallatens B. et Gill. - Tarn (H. Sudre).

291 - R. obvallatus B. et Gill. var. uncinulatus. - Sarthe (Coilliot).

Foliole caul. terminale cuspidée; dents divariquées, aiguillons pétiolaires crochus et nombreux.

295 - R. olbvallatus B. et Gill. var. uncinulatus. - Sarthe (Henry).

296-R. Nouletii Sud. - Hte-Garonne (H. Sudre).

Turion glabre, non pruineux ; inflorescence pauciflore, armée, glanduleuse ; fleurs d'un rose vif, à carpelles glabres. Pour le reste, voisin du $R$. obvallatus B. et Gill.

\section{$c$ - Imbricati Sud.}

Q98 - IR. HMIBMCATUS Hort. v. virescens Sud. - France (H. Sudre).

Plante née en 1902 et bien normale depuis deux ou trois ans. Ne diffère en rien de celle d'Angleterïe; elle a une tendance à devenir un peu discolore, caractère qui s'accentuera apparemment avec l'âge. - Je compléterai ce $n^{\circ}$ ultérieurement.

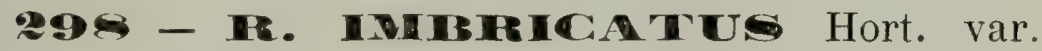
subrotundus Sud. - France (H. Sudre).

Plante née en 1902, ayant bien conservé tous ses caractères; a un peu le port des $R$. Triviales P.-J. Müll. - Ce $n^{0}$ sera complété ultérieurement. 
299 - IE. IMTEICA TUE Hort. var. hamulosus Sud. - France (H. Sudre).

Plante née en 1902. Elle ressemble beaucoup à la forme sauvage de Vendée, mais est moins discolore; il est probable que le tomentum s'accroîtra avec l'âge.

Cette var. est très rapprochée de la var. cariensis (Rip. et Gen.), qui dérive certainement aussi du R. $i m$ bricatus Hort.

300 - Tr. Hecosnitras Sud. - Tarn (H. Sudre).

Se rapproche du $R$. imbricatus Hort. En diffère par ses aiguillons moins forts, ses feuilles plus finement dentées, à folioles moins larges, la terminale plus longuement pétiolulée, son inflorescence pauciflore et son réceptacle glabre. Plante très précoce, intermédiaire entre les $R$. imbricatus Hort. et cardiophyllus L. et M.

226 - On trouvera dans ce fascicule quelques échantillons de $R$. brachythyrsoides provenant de mes cultures et destinés à compléter ce $\mathbf{n}^{0}$ (fasc. V).

Toulouse, le 10 Novembre 1908.

\section{H. SUDRE.}





\section{RUBI RARI VEL MINUS COGNITI EXSICCATI}

Je possède, à un très petit nombre d'exemplaires, des types authentiques de quelques espèces de Müller et de Lefèvre et de quelques autres Rubus décrits lans mes Diagnoses de Rubus nouvecux ou ailleurs. Je me propose de les distribuer par fascicules qui paraîtront à peu près ì la mème époque que les Rubi Europoe. Le prix en sera évalué à raison de $0 \mathrm{fr} .50$ par numéro. La collection complète comprendra environ de 120 à 150 numéros répartis en 5 ou 6 fascicules.

A part de très rares exceptions relatives à quelques types authentiques, les plantes des $R u b i$ rari ne figureront pas dans le $B$ atolneca.

Cornme je ne puis disposer que de 4 ou 5 exemplaires de cette nourelle collection, les botanistes qui désirent la recevoir complète feront bien de m'en aviser le plus tôt possible.

\section{H. S.}

\section{GOMPOSITION DU FASG. I}

1. - R. hamulosus L. et M.! 12. - R. Libertianus Vh.

2. - R. Rogersii Lint 13. - R. Vallicolus Müll.!

3. - R. carpinifolius v. laxus. 14. - R. chloophyllus Sud.

4. - R. Salteri Bab.

5. - R. latifolius Bab.

15. - R. dumnoniensis V. eupectus Sud.

6. $-\times R$. exhaustus Sud. 16. - R. fuxeensis Sud.

7. $-\times$ R. doburiensis Sud. et 17. - R. orbifer Sud. LeV.

18. - R. silvicolus M. et L.!

8. - R. immutabilis Sud.

19. - R. hirsutissimus Sud. et

9. - R. orbifolıatus Sud. Ley.

10. - R. stenoacanthus M. etL.! 20. - R. patuliformis Sud.

11. - R. belophorus M. et L. !

Prix : 10 frances (port en sus)

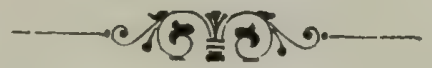





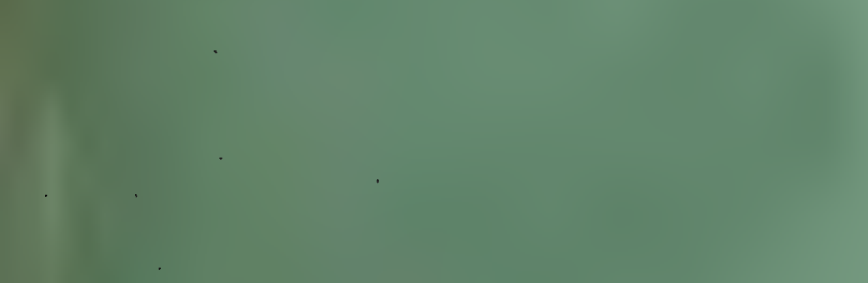

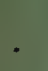

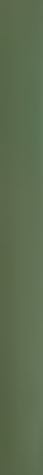







\title{
BATOTHECA EUROP龙A
}

PUBIIE PAR

\author{
I. SUD RE \\ Professeur à l'Ecole normale de Toulouse (France) \\ 12, rue André Délieux \\ FASCICULE VII - 1909 \\ $\rightarrow .9 * 6$
}

Paraît par fascicules annuels de 500 numéros. Les collaborateurs qui fournissent $\check{5}$ numéros convenus d'avance et préparés à 25 parts, reçoivent un fascicule en échange. Chaque exemplaire doit compren ire, autant que possible, deux rameaux, l'un florifère et l'autre fructifère, plusieur's feuilles caulinaires et quelques pétales préparés à part. Les espèces déjà distribuées ne sont admises que si elles diffèrent quelque peu des formes publiées.

\section{Prix du fascicule : $\mathbf{2 5} \mathbf{f r}$.}

\section{COLLECTEURS POUR LE $7^{\circ}$ FASCICULE}

Dr Bouly de Lesdain, à Dunkerque (Nord), no 319.

G. Bouvet, pharmacien à Angers (Maine-et-Loire), no 308.

$\times$ Brochon. Bordeaux, no 350 .

Abbé Charbonnel, La Chapelle-Laurent (Cantal), no 341.

Coilliot, au Mans (Sarthe), n ${ }^{\circ} 338$.

Duffort, pharmacien à Masseube (Gers), no 304 .

Dr Gillot, Autun (Saône-et-Loire), no 347.

$\times$ Levent (Marne), no 322 .

G. Maire, ingénieur à Alexandrie, no 331.

$\times$ P.-J. Müller (Alsace), n²32.

$\times$ Questier, Thury en Valois, nos $30 \%, 339,342,344$.

H. Sudre, Toulouse, nos $301,302,305,309-312,314,315,319-321,323$ $330,333337,340,343,345,348,349$.

$\times$ Timbal-Lagrave, Toulouse, nos $313,316,318$.

$\times$ Tourlet, Chinon (Indre-et-Loire), no 346.

Major Wolley-Dod, Walton-on-Thames (Angleterre), no 303 . .

\section{OBSERVATIONS}

Les collaborateurs sont priés de ne pas récolter les espèces déjà publiéeś dans le Butotheca. - Ce fascicule renferme 23 Rubus non distribués dans des exsiccata numérotés. Le fasc. VIII sera consacré aux $R$. discolores et aux $R$. vestiti.

H. SUDRE. 
Sect. II. - Silvatici P.-J. Müll.

Gr. $b$ - Euvirescentes Gen.

301 - R. tolosanus Sud. - Hte-Garonne (H. Sudre).

C'est en faisant des recherches à la ferme de Bégué dans le but de retrouver le Rosa Clotildea Timb. décrit par Déséglise, que j'ai rencontré ce Rubus. Je l'avais vainement recherché jusqu'à cette année. La plante croît à une centaine de mètres de la forêt de Bouconne, en pleine lumière, et a une tendance à devenir discolore; il y aurait peut-être lieu de la rapprocher du $R$. argenteus W. N. Ses fleurs sont très grandes et atteignent $0 \mathrm{~m} .035$ de diamètre. Elle est abondante dans cette localité. T'ient à la fois des $\boldsymbol{R}$. fagicola, macrophyllus et consobrinus.

302 - R. debilispinus Sud. var. ligerinus. - France (H. Sudre).

Plante née en 1902 et aujourd'hui très normale. A les aiguillons plus robustes que les autres formes de ce groupe; du reste c'est une espèce silvatique que je cultive en pleine lumière, aussi ses aiguillons sont-ils plus forts que dans la plante sauvage ; elle reste toutefois assez nettement virescente. La plante est originaire du bois de Torfou, en Maine-et-Loire, où je l'ai récoltée en 1901.

Gr. $c$ - Discoloroides Gen.

SOS - MR. TNDLEYANUS Lees. Angleterre (Wolley-Dod).

301 - R. Duffortii Sud. - France (Duffort).

Remarquable par la brièveté de ses aiguillons caulinaires.

3O5 - IR. ACTER NIFCGRUG M. et L. - France (H. Sudre).

Est originaire de Châteaulin (Finistère) et âgée de 7 ans. Le buisson est encore peu vigoureux et je compléterai ce $\mathrm{n}^{0}$ ultérieurement. 
306 - IE. REIATINIFOLIUSA W. N. Allemagne.

Ce Rubus était destiné aux Rubigallici ; il me fut donné par $\mathrm{M}$. le $\mathrm{D}^{\mathrm{r}}$ Bouly de Lesdain, après la mort de l'abbé N. Boulay. Malheureusement la plante était sans étiquette. Comme elle était logée dans du papier de journaux allemands portant la date de juin 1897, il est à peu près certain qu'elle a été cueillie en Altemagne, mais j'ignore par qui. Comme elle est très typique, il est possible qu'elle provienne des environs de Minden.

307 - R. CARDIOPHYLLUS Lef. et M.! - Valois (Questier).

Sous-espèce bien tranchée reliant les $R$. silvatic aux $R$. discolores.

Sect. III. - Discolores P.-J. Müll.

Gr. a - Gypsocaulones P.-J. Müll.

308 - R. bellidiflorus C. Koch. - Angers (G. Bouvet).

Le buisson n'ayant pas mis de turions cette année, les feuilles caulinaires seront distribuées ultérieurement.

309 - Te.peduncularis Timb. - Toulouse (H. Sudre).

Ordirairement les feuilles caul. sont plus amples et plus manifestement acuminées.

310 - TE. subtruncatus Sud. var. glaphyrus. - France: Ariège (H. Sudre).

311 - TR. Subtruncatus Sud. var. anoplothyrsus. - France: Ariège (H. Sudre).

Pour les caractères, voir Rubi Europa p. 70.

$3 \geq-R$. argillaceus Sud. - France : Albi (H. Sudre).

Le pollen n'est yas pur comme chez les autres formes dugr. du R. ulmifolius schott; toutefois la plante 
fructifie assez bien. Elle pourrait dériver par croisement du $R$. perconspicuus, qui sera distribué prochainement; toutefois cela me paraît peu probable, ce dernier croissant à $3 \mathrm{~km}$. environ du $R$. argillaceus.

313 - R. serriculatus Rip. var. tiliffolius. France (Timbal-Lagrave).

Se rapproche de la var. apiculifer du $R$. vulgatus Sud.

31 - R. insignitus Timb. et Müll. France (H. Sudre).

Ces spécimens sont identiques au type de TimbalLagrave, récolté aux environs de Toulouse !

315 - Fe. insignitus Timb. et Müll. var. dispalatus Sud. - France (H. Sudre).

316 - R. insignitus Timb. et Müll. var. dispalatus Sud. - France (Timbal-Lagrave).

Plus typique que le $n^{\circ} 315$. Forme commune dans les Pyrénées.

\$1 7 - T. amisodon Sud. - France: Nord (B. de Lesdain).

-18- IR. anisodon Sud. var. Bastardianus (Gen.) - France (Timbal-Lagrave).

319 - Re. misodon Sud. var. pronatiflorus Müll. et Timb. - France : Toulouse : (H. Sudre).

Inflorescence très armée, à pédoncules très étalés. Je l'ai récolté dans la localité où Timbal avait cueilli ses spécimens.

$\mathbf{3 2 0}-\mathbf{R}$. albidiflorus Sud. - France : Hte-Garonne (H. Sudre).

321 - IR. vulgatus Sud. var. apiculatifolius. - France (H. Sudre).

Forme très vigoureuse, se rapprochant un peu du R. subtruncatus Sud. var. glaphyrus (Rip.) 
$\mathbf{3} \mathbf{2}-\mathbf{R}$. cuneatus Boul, et Bouv. var. ischnocicanthus Sud. - France : Marne (Levent).

Se rapproche du $R$. serriculatus Rip. par sa dentelure très fine.

323 - He. heteromonphus Rip. var. subcunealus Sud. - France: Tarn (H. Sudre).

Diffère du type par son tomentum ras et ses anthères un peu poilues. Faciès du $R$. cuneatus $\mathrm{B}$. et $\mathrm{Bv}$.

38 - R. Lemaitrei Rip. var. cognobilis (Müll. et Timb.) ! - France: Toulouse (H. Sudre).

$\mathbf{3} \mathbf{2}-\times$ R. SEMITOLOSANUS Sud. ; $R$. ulmifolius $\times$ tolosanus. - France $(H$. Sudre).

Le tomentum et la coloration vive des fleurs sont du $R$. ulmifolius ; rappelle le $R$. tolosanus par les glandes sessiles de ses turions, la forme de ses folioles caulinaires et ses axes florifères poilus. Le $R$. tolosanus est abondant dans cette localité.

326 - $\times$ R. DENSIFLORENS Sud. ; $R$. ulmifolius XGillotii - Tarn : Le Fraïssé (H. Sudre).

Beaucoup plus rapproché du $R$. ulmifolius Schott que du $R$. Gillotii N. Boul. N'a de celui-ci que les grands aiguillons, la denticulation vive et la pubescence de la face inférieure des feuilles. Plante stérile, à pollen atrophié. Les 2 parents sont abondants dans le voisinage de l'hybride.

32 - R. PULVERULFNTUS Sud. ; $R$. ulmifolius Xtomentosus. - Toulouse (H. Sudre).

Voisin du suivant, mais feuilles tomentelleuses en dessus.

$\mathbf{B} 8-\times R$. NOTHUS Sud. ; $R$. ulmifolius $\times$ tomenlosus Lloydianus. - Tarn (H. Sudre).

Port, feuilles 5̃-nées, styles rouges, carpelles velus du $R$. ulmifolius ; mais axe florifère poilu, aiguillons faibles, pétales blancs, étroits, du $R$. Lloydlianus Gen. Quelques fleurs fructifient et d'antres avortent. 
$329-X$ R. GIRAUDIASII Sud. ; R. ulmifolius $X$ pallidiformis. - Ariège (H. Sudre).

$330-X R$. VALDELAXUS Sud. - France: Tarn (H. Sudre).

L'influence du $R$. ulmifolius n'est pas douteuse; quant à l'autre parent il ne m'est pas possible de préciser.

331 - R. SANCTUS Schreb. - Egypte (G. Maire).

M. G. Maire, ingénieur à Alexandrie, qui a eu l'extrême obligeance de me récolter ce Rubus, m'a transmis les renseignements suivants: les feuilles caulinaires sont toujours 3-nées; la plante forme des buissons épais et très volumineux et est difficile à observer de près car les touffes centrales florifères sont rendues presque inabordables par l'entrelacs des turions. J'ai vu de nombreux spécimens de $R$. sanctus provenant de Bosnie, d'Hercégovine, de Corcyre et du Caucase ; en général la plante bien qu'ayant le pollen mélangé, fructifie mieux que celle que je distribue, dont la stérilité partielle est sans doute due à l'aridité de la station.

33: - TR. GODREONIL L. et Lmt. - Alsace (P.-J. Müller).

Quelques rameaux, cueillis sans doute à l'ombre, sont presque virescents; la plante est ordinairement discolore.

333 - A. amplistipulus Sud. - Pyrénées (H. Sudre).

334 - R. WINTERI P.-J. Müll. - France: Puy-deDôme (H. Sudre).

La forme du centre de la France, qui est le $R$. Rivulii B. et Q. ( $=R$. serratus B. et Let.), est moins robuste que celle des Pyrénées (Bat. eur. $\left.n^{\circ} 16\right)$.

335 - R. WINTERI P.-J. Müll. var. rhombeus. France : Tarn (H. Sudre).

Foliole caul. terminale rhombée ; dents grosses, inflorescence feuillée. 
336 - Te. ellipticifolius Sud. - France: Ariège (H. Sudre).

338 - PROPINQUUS P.-J. Müll. - France : Puy-deDôme (H. Sudre).

338 - R. PPOPINQUUS P.-J. Müll. var. immitis (Bor.) - France : Sarthe (Coilliot).

339 - R. PROPINQUUS P.-J. Müll. var. dumosus (Lef.) - France : Valois (Questier).

Cette plante, bien que soumise à Müller par cefèvre, ne fut pas décrite dans le Versuch; Müller l'étiqueta: "R. propinquus? ". La glaucescence n'est pas toujours apparente sur les turions, mais je l'ai observée d'une facon bien manifeste sur un grand nombre d'échantillons. Cette variété se rapproche du $R$. Godronii L. et Lmt. sous certains rapports.

$$
\text { Gr. } b-\text { Hedycarpi (Hocke). }
$$

310 - Re. HIFONA Vest. - France : Tarn (H. Sudre).

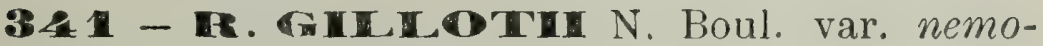
philus (Rip.) - France: Cantal (Charbonnel).

$3 \mathbf{2} \mathbf{2}$ - R. stenopetalus Lef. et M. 1 - France : Valois (Questier).

343 - R. GENTCUTATUS Kalt. France : Puy-de-Dôme (H. Sudre).

3ha - TE. GENTCUTATUG Kalt. France: Valois (Questier).

Spécimens authentiques de $R$. cerasifolius L. et M. ! correspondant exactement au $R$. geniculatus Kalt.

345 - R. goniophylloides Sud. - France : HteGaronne (H. Sudre).

Intermédiaire entre $R$. geniculalus et thyrsoideus (goniophyllus). 
$3 \mathbf{8} 6-\times$ R. ROBUSTIFRONS Sud.; $R$. procerus $\times$ ulmifotius. - France : Indre-et-Loire (Tourlet).

Très rapproché du $R$. procerus var. robustus, mais un peu de glaucescence, inflorescence multiflore ; stérile.

3 - 7 - $X$ R. CHNOOPHYLLUS P.-J. Müll.; $R$. procerus $\times$ tomentosus. - France : Saône-et-Loire (Gillot).

A les feuilles moins nettement tomenteuses en dessus que la plante type de Nancy, et les turions un peu velus, mais je crois que l'origine en est la même.

$3 \mathbf{A}$ - R. occiduus B. et Bouv. var. curtipes Sud. France : Tarn (H. Sudre).

Intermédiaire entre $R$. procerus P.-J. Müll. et $R$. Questieri L. et M. Comme il est bien fertile et très abondant je ne puis croire qu'il en soit hybride.

329 - IR. Iacertosus Sud. - France : Tarn (H. Sudre).

D'abord subordonné au $R$. thyrsoideus Wimm., mais plus rapproché, sous ses formes typiques, du R. procerus P.-J. Müll. Ce $n^{\circ}$ représente une forme aprique, ayant végété sur un terrain aride.

350 - R. Iacertosus Sud. - France : Gironde (Brochon).

Forme très vigoureuse, ayant poussé sur un terrain très fertile.

Toulouse, le 5 Novembre 1909.

H. SUDRE. 




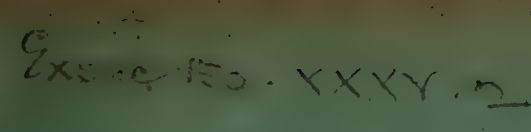

H. Sudre

Batatheca Europaiea

fase VIII.

$$
1910
$$





\section{BATOTHECA EUROP㾁A}

PUBLIÉ PAR

\section{H. SUDRE}

Professeur à l'Ecole normale de Toulouse (France)

12. rue André Délieux

FASCICULE VIII - 1910

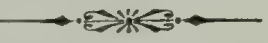

Parait par fascicules annuels de כొ0 numéros. Les collaborateurs qui fournissent 5 numéros convenus d'avance et préparés à 2ö parts, recoivent un fascicule en échange. Shaque exemplaire doit comprentre, autant que possible, deux rameaux, l'un florifere et l'autre fructifère, plusieur's feuilles caulinaires et quelques pétales préparés à part. Les espèces déjà distribuées ne sont admises que si elles diffèrent quelque peu des formes publiées.

\section{Prix du fascicule : $\mathbf{2 5} \mathbf{f r}$.}

\section{COLLECTEURS POUR LE $8^{\circ}$ FASCICULE}

C. Bioknell, à Bordighera (Italie), no 393,

G. Bouvet, pharmacien à Angers (Maine-et-Loire), "no 354.

Brevière, à Arlanc (Puy-de-Dôme), no 382.

Charbonnel, à La Chapelle-Laurent (Cantal), nos 352, 398.

$\times$ Chaboisseau, Pindray (Vienne), nos $372,389$.

Coilliot, au Mans (Sarthe), n 357 .

A. Gentil, au Mans (Sarthe), no 366 .

$\times$ G. Genevier, Mortagne (Vendée) no 381.

A. Henry, au Mans (Sarthe), nos 351, 388, 399.

E. Holzfuss, à Stettin (Poinéranie), no 365 .

D' Kinscher, à Peterwitz (Silésie), n $36 \%$.

$\times \mathbf{P}$.J. Mủller, à Wissembourg' (Alsace), $110 \mathrm{~s} 369,3 \% 0$.

$\times$ Questier, à Thury-en-Valois, nos 364, 396.

Spribille, prof. à Bieslau (Silésie), no9 362, 371 .

H. Sudre, i Toulouse, $11^{\text {กs }} 353,355,358-60,373-80,383-7,390-2$, $394-5,397,400$.

$\times$ Timbal-Lagrave, à Toulouse, no 361 .

Zinsmeister, Wengen (Baviere), nos $356,363,368$.

\section{OBSERVATIONS}

Les collaborateurs sont priés de ne pas récolter les especes déjà publicies dans le Batotheox. - Ce fascicule renferme 32 Rubus non distribués dans des exsiccata numerotés. Le fasc. IX seraconsacré aux $R$. vestiti et aux $R$. Rudulo.

H. SUDRE。 
Sect. III. - Discolores P.-J. Müll.

Gr. b. - Hedycarpi Focke.

351 - R. Iopidug P.-J. Müll. - France : Angers (Henry).

$352-\times$ R. LATIDENTATUS Sud.; $R$. evagatus $\times$ Lloydianus. - France : Cantal (Charbonnel).

Se ranproche du $R$. polyanthus P.-J. Müll. (candicans $\times$ Lloydianus $)$ mais le turion un peu pubescent, les folioles intérieures pétiolulées, l'inflorescence très armée rappellent le $R$. evagatus.

353 - Te omollitus Sud. - France: Luthon (H. Sudre).

Plante commune dans les vallées des Pyrénées centrales.

351 - Re. Omollitus Sud. var. confluentinus (Wirtg.). - France : Maine-et-Loire (Bouvet).

A été recueilli au Jardin des Plantes d'Angers d'un pied provenant de la Membrolle. Se rapproche un peu du type $R$. pubescens Wh., mais est plus voisin du $R$. emollitus.

355 - IR. adumcispinue Sud. - France (H. Sudre).

Ronce née en 1902, aujourd'hui très vigoureuse. Ne diffère en rien de la plante sauvage du bois de Torfou (Maine et-Loire).

356 - Te. PreOCARUS Müll. var. arrigentiflorus Sud. - Bavière (Zinsmeister).

Inflorescence du $R$. arrigens Sud. (Bat. no 24) mais turion obtus, foliole caul. terminale moins large, obovée, pollen moins imparfait.

35 7 - R. occiduus B. et Bv. var. Coilliolii Sud. - France : Le Mans (Coilliot).

Pollen très imparfait; fructifie assez bien, comme le type; les fruits sont tombés sur quelques rameaux. 
$358-\times R$. BOSQUETIANUS Timb. et Müll.; R. lacerlosus $\times$ ulmifolius Sud. - France: HauteGaronne (H. Sudre).

Sans être tout à fait identique à la plante type de St-Urcisse (Tarn), elle a certainement la même origine.

359- $-\times$ R. PSEUDO-SIMILLIMUS Sud. ; $R$. lacertosus $\times$ tomentosus. - France : Hte-Garonne $(H$. Sudre).

Feuilles du $R$. lacertosns, mais les supérieures nettement tomentelleuses en dessus; turion plus faible; tleurs blanches, à pétales larges.

\section{Gr. c. - Candicantes Focke}

360 - R. phyllostachys P.-J. Müll. France: Tarn (H. Sudre).

E61 - Re. phyllostaohys P.-J. Mùll. France: Tarn-et-Garonne (Timbal-Lagrave).

362. - Rt. phyllostachye P.-J. Müll. var. incisiserratus spribille! - Silésie (Spribille).

Feuilles moins amples que dans le type; dentelure grossière et très irrégulière ; folioles courtement pétiolulées, fleurs petites. Turion nettement canaliculé, \pm pubescent.

363-R. Leventii Sud. - Bavière (Zinsmeister).

Voisin du $R$. phyllostachys Müll., mais folioles terminales rhombées ou obovées.

36- R. aciodontus Lef. et M. ! - France : Valois (Questier).

Dérive peut-être des $R$. stenopctalus L. et M. et propinquis v. dumosus? 'Toutefois fructifie bien.

365 - Tr. Ilnyromibus Focke. - Poméranie (Holzfuss).

366 - 16. thyosamthus F. v. hylophilus (Rip.) - France: Sarthe (Gentil). 
Feuillage moins ample que dans $R$. thyrsanthus; carpelles velus.

367 - Fe. constrictus L. et M. - Silésie (Kinscher).

Intermédiaire entre $R$. sulcatus Vest et $R$. Ihyrsoideus Wimm., mais non hybride.

368 - R. constrictus L. et M. var. rose $i$ florus. - Bavière (Zinsmeister).

Inflorescence très feuillée; fleurs roses, à étamines dépassant à peine les styles; dents très aiguës.

369 - Th. candicane Wh. var. patulipes Sud. - Alsace (Müller).

Inflorescence dense, à pédoncules étalés ; feuilles de la var. coarctatus.

370 - 18. candicans Wh. var. roseolus (P.-J. Müll.) ! - Alsace (Müller).

Il me parait difficile de voir dans cette forme autre chose qu'une variété du $R$. candicans Wh. Les caractères du $R$. roseolus donnés dans la Flore de France: "Turion moins robusle, moins canaliculé sur les faces, glabre, garni d'aiguillons.s médiocres ou petits... Rameau... cylindrique..." ne s'appliquent guère à ces spécimens, qui constituent pourtant le type de Muller et qui me paraissent à peu près identiques à la plante des Ronces rosgiennes. J'ajouterai que le $\mathrm{n}^{0}$ t99 de l'Assoc. rubol. n'est pas un $R$. roseolus mais bien le $R$. excelsior Müll., et que le n" 622 est à rapprocher du $R$. thyrsanthus F. var. argyropsis.

371 - Re. candicans Wh. var. aculiserratus Sprib. - Silésie (Spribille).

Inflorescence du $R$. pubescens Wh. mais turion presque glabre.

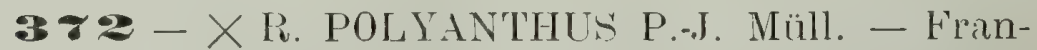
ce: Vienne (Chaboisseau).

Pappelle le $R$. candicans par la forme de son inflorescence; le tomentum des feuilles est celui du R. Lloydianus. Peu fertile. 
Gr. d. - Subtomentosi Sud.

$\mathbf{3} \mathbf{8 3}$ - R. vicarius Sud. - France : Tarn (H. Sudre).

Commun dans la région montagneuse du 'Tarn, où il remplace le $R$. arduennensis Lib.

$\mathbf{3} \mathbf{A}-\mathbf{R}$. vicarius sud. - France (H. Sudre).

Ronce née en 1905, aujourd'hui très normale. Ne diffère en rien de plante sauvage. Si les $R$. sublomentosi sont des hybrides, il faut reconnaître qu'ils se comportent comme de bonnes espèces pures.

385 - R. malacus Sud. v. albiflorus. - France (H. Sudre).

376 - R. malacus Sud. v. roseiflorus. - France (H. Sudre).

37 - Ie. moestus Hol. var. pulvereus Sud. France : Tarn (H. Sudre).

Très fertile; pollen très peı mélangé. Le type mostu.s dérive peut-être du $R$. procerus Müll., mais ce n'est pas le cas du $n^{0} 37 \%$.

378 - Re. collicolug Sud. - France : HteGaronne (H. Sudre).

389- TR. collicolus sud. var. crosulus. France: Albi (H. Sudre).

Turion canaliculé ; folioles étroites, à dents larges, irrégulières ; fleurs grandes.

880 - 35. subvillosus Sud. v. albiftomes. France: Haute-Garonne (H. Sudre).

381- Te. Gubvillowins sud. v. vendeunus (Gen. !). - France : Deux-Sèvres (Genevier).

spécimens authentiques, préparés pour los exsiccata de Müller, qui ne parurent jamais. 
Sect. IV. - Appendiculati Gen.

Gr. a. - Tomentosi Wirtg.

382 - TR. TOMENTOSU Borkh. France : Puy-de-Dôme (Brevière).

383 - R. TOMNNTOSUS Borkh. var. arvicolus Sud. - France: Haute-Garonne (H. Sudre).

Pour la description, voir mes Rubi Europee p. 98.

384 - Re. TOMENTOSU Borkh. var. densus Sud. - France : Haute-Garonne (H. Sudre).

Diffère du 383 par ses folioles plus larges, ses turions glabres, ses rameaux églanduleux, ses pétales moins larges, son pollen plus pur.

385 - X R. TOMENTELLIFOLIL'S Sud.; R. tomentosus $\times$ ulmifolius. - Haute-Garonne (H. Sudre).

La forme distribuée se rapproche beancoup du R. proximellus Rip., sans être tout à fait identique.

386 - R. tomenticaulis Sud. - Toulouse (H. Sudre).

Pilosité des turions caractéristique. Croît en plein bois.

387 - R. tomsntosifrons Sud. - Toulouse (H. Sudre).

Distinct du R. tomenticaulis par ses larges folioles, ses pétales largement ovales, son inflorescence églanduleuse.

$\mathbf{3 8 8}-\times$ R. ROSEIPETAlUS sud. - France: Sarthe (Henry).

Provient apparemment du $R$. vulgatus, qu'il rappelle par la forme des folioles terminales.

389 - $\times$ R. ROSEIPETALUS fa $R$. splendens Chab. 1 - France: Vienne (Chaboisseau).

Produit apparemment par le $R$. congustifactus sud. Spécimens authentiques! 
390 - R. subparilis sud. - f'rance : Hautc-(jaronne (H. Sudre).

Est au $R$. Lloydianus Gen, ce que le $R$. tomenticrulis est au $R$. tomentosus.

391 $-X$ R. MARNACEUS sud. - France: Tarn (H. Sudre).

Turion du R. subpurilis, mais glandes nulles, port du $R$. lacerlosus. Stérile.

392 - R. ancophilus Sud. - France: Tarn (H. Sudre).

Plante rare. Je distribuerai des rameaux florifères plus tard, quand je pourrai en cueillir.

393 - Re. ancophilus sud. var. Bicknellii (Burn. et Grl.) - Italie : Ligurie (Cl. Bicknell et Pollini).

Se rapproche du $R$. incanescens, mais le turion n'est pas glauque, la dentelure des feuilles est très grossière.

394 - R. INCANESCENS Bert. - France: Tarn (H. Sudre).

Croit avec $R$. ancophilus. 'Turion glauque, dentelure plus fine; inflorescence plus glanduleuse.

395 - $\times$ R. BERTOLONII Sud. - France : Tarn (H. Sudre).

Certains rameaux fructifient assez bien et d'autres sont tout à fait stériles, et cela sur un même buisson. Faciès du $R$. incanescens, mais glandes à peu près nulles, fleurs roses, aiguillons égaux. Est assez commun dans cette localité, où les parents sont fréquents.

$$
\text { Gr. b. - Vestili Focke. }
$$

396; - R. leucanthemus P.-J. Müll. - France (Questier).

C'est l'excédant de la centurie récoltée pour les exsiccata de Billot ( $\left.\mathrm{n}^{0} 2450\right)$. dre).

397 - - R. pilifer sud. - France: Tarn (H. su-

Intermédiaire entre $R$. leucunthemus et $R$. allscilus. 
Peut-être hybride fixé de $R$. leucanthemus et de $R$. Lloydianus. Toutefois, dans le Tarn, le $R$. leucanthemus manque là où le $R$. pilifer croît en abondance. nel).

$\mathbf{3 9 8}$ - R. pilifer Sud. - France : Cantal (Charbon-

Ne semble pas différer du $n^{0} 397$; toutefois paraît mal fructifier. Il m'est arrivé de rencontrer sur le Plateau Central le $R$. leucanthemus, d'habitude très fertile, tout à fait stérile : cette stérilité est sans doute accidentelle. Le $R$. pilifer est commun en Auvergne.

$399-\times$ R. ADRIENII Sud. - France : Sarthe (Henry).

Ressemble un peu au $R$. propinquus v. macrobelophorus, mais est stérile et porte quelques rares glandes.

G00 - R. perconspicuns Sud. - France: Tarn (H. Sudre).

Plante discolore, intermédiaire entre $R$. argenteus et $R$. vestitus. Localité unique.

305 - On trouvera dans ce fasc un supplément pour le $\mathrm{n}^{\circ} 305$ ( $R$. alterniflorus).

Toulouse, 25 octobre 1910.

H. SUDRE.

Albi_-Impr. A. Nougutês, rue de l'Hôtel-de-Ville, 33 


Exsed 450. XXXY.2

Yl. Sndre

Batotheca Europiâa.

fasc. Ix. 1911 



\section{BATOTHECA EUROPEA}

PUBIIE PAR

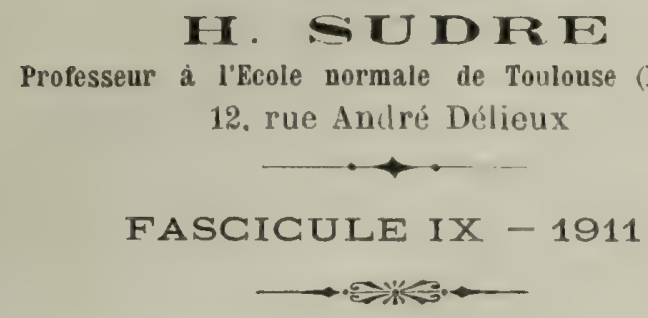

Parait par fascicules annuels de 50 numéros. Les collaborateurs qui fournissent כ̆ numéros convenus d'avance et préparés à 20 parts, recoivent un fascicule en ichange. thaque exemplaire doit comprendre, autant que possible, deux rameaux, l'un florifere et l'autre fructifere, plusieur's feuilles caulinaires et quelques pétales preparés a part. Les espèces déja distribuées ne sont admises que si elles different quelque peu des formes publiées.

\section{Prix du fascicule : $\mathbf{2 5}$ fr.}

\section{COLLECTEURS POUR LE 9' FASCICULE}

Dr Biau, à Bruyères (Vosges), nos 427, 444 ,

Dr Bouly de Lesdain, à Dunkerque (Nord), no 426.

$\times$ Chaboisseau (Vienue), nos 405, 408.

Charbonnel, à La Chapelle-Laurent (Cantal), nos 421.

K. Fritsch, à Gratz (Styrie), n* 423 .

$\times$ Gravet (Belgique), nos 404, 432.

A. Henry, Le Mans (Sarthe), nos $409,440,446$.

H. Hofmann, à Grossenhain (Saxe), no 439.

$\times$ Levent (Marue), no 431.

$\times$ P.-J. Müller, à Wissembourg (Alsace), nos 402.

A. Ninck, à Bar-le-Duc (Meuse), $11^{0} 401$.

Du Pré (Belgique), nos 415.441.

$\times$ Questier, il Thury-c11-Valois, $1105416,417,419,420,421,425,445$, $447,448$.

Dr Sabransky, à Sœchau (Styrie), nos $407,422$.

Spribille, prof. à Breslau (Silésie), nos 430.

H. Sudre, à Toulouse, $10^{0 \mathrm{~s}} 406,410-12,414,418,428,429,433,431$, $436,437,438,442,443,449,450$.

$\times$ Timbal-Lagrave, à Toulouse, no 413.

Wolley-Dod, à Walton-on-Thanes (Angleterre), no 403.

\section{OBSERVATIONS}

Les collaborateurs sont priós de ne pas récolter les espèces déja publiées dans le Britothere. - ('e fascocule renferme 31 Rubus non distribués dans des exsiccata numérotés. Le lasc. X sera consacré aux $R$. Raduloe.

H. SLDRE. 
Sect. IV. - Appendiculati Gen.

B - Vestiti Focke

a. - Hypolenci Sud.

No 101 - Re. conspicuus P.-J. Müll. France: Meuse (A. Ninck).

No $\mathbf{1 0}-\times$ R. CERINOSTYLUS P.-J. Müll. ! Alsace (P.-J. Müller).

Spécimens authentiques.

No 203 - R. Wolley-Dodii Sud. - Angleterre (Wolley-Dod).

Voisin du R. macrostachys Müll. mais inflorescence bien plus armée, dentelure plus fine, fleurs blanches.

№ $\mathbf{2}$ - $\mathrm{R}$. infrarugosus Sud. - Belgique (Gravet).

No 105 - Mt. septoram P.-J. Müll. France : Vienne (Chaboisseau).

Spécimens authentiques préparés par Chaboisseau pour les exsiccata de Müller.

No $\mathbf{1 0 6}$ - R. clethrocharis Sud. - France : Tarn (H. Sudre).

No $\mathbf{0}$ - R. styriacus Hal. - Styrie (Sabransky).

No $\mathbf{1 0 8}$ - Tr. Saxigenus var. BELOSTACHYS Sud. - France : Vienne (Chaboisseau).

Voir les observations de Chaboisseau jointes aux spécimens. C'est peut-ètre un $R$. Chaboisseauiメulmifolius?

No 09 - R. BOREAUANUS Gen. - France: Sarthe (Henry).

No $10-R$. secedens Sud. - France : Vendée (H. Sudre). 
Plante née en 1902. Identique ì la forme sauvage de Mortagne, murs du village.

$\mathrm{N}^{\circ} 1-\mathrm{R}$. continens Sud. - France : Ariège (H. Sudre).

No $4 \geq$ - Re. demsipilus Sud. - France : Hte-Garonne (H. Sudre).

Intermédiaire entre $R$. Chaboisseaui Müll. et $R$. adscilus Gen. Commun dans la région toulousaine.

No 13 - 13 R. PSEUDO-CONFORMIS Sud. France : Hte-Garonne (Timbal).

Ne dérive pas du R. conformis, ainsi que je l'avais nensé tout d'abord, car le $R$. conformis manque à Balma. Je n'ai pu retrouver cet hybride à Balma.

No $1-\times R$. SFMIDENSIPILUSSud. - Hrance: Hte-Garonne (H. Sudre).

Etait presque stérile en 1908, mais a fruciifié partiellement en 1909. Se rapproche du $R$. macros/achys Müll. var. scabridus Sud. Rub. Eur., mais je le crois dérivé des $R$. densipilus et lacerlosus (roseiflorus) au milieu desquels il croît.

\section{b - Virescentes Sud.}

No $15-R$. Prei Sud. - Belgique (du Pré).

II. Du Pré avait récolté de nombreux spécimens de cette plante, qu'il rapportait avec doute an $R$. subcanus Miill. Quelques exemplaires avaient été appelés R. Menkei par M. Focke.

No $1-R$. truncifatus Sud. - France : Aisne (Questier).

Feuilles moins finement dentées que dans les autres formes de ce groupe.

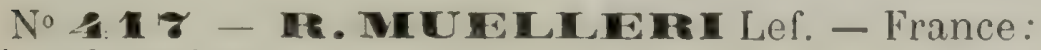
Oise (Questier).

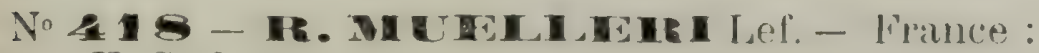
Tarn (H. Sudre). 


\section{$-104-$}

Plante très précoce. J'ai cueilli les rameaux florifèressur un pied que j'ai planté dans mon jardin.

No 19 - R. grypoacanthus L. et M. 1 - France: Aisne (Questier).

Aiguillons caractéristiques.

No 420 - R. splendidns M. et Lef. 1 France : Aisne (Questier).

Mériterait d'ètre étudié sur place ; c'est peut-être un hybride de $R$. rudis et de quelque forme à fleurs roses. Les rameaux fructifères que j'ai vus portaient des fruits assez bien développés.

No 23 - IR. COLEMANNAI Blox. France : Cantal (Charbonnel).

Me paraît à peu près conforme an type de Bloxam, de Coventry, type qui a disparu aujourd'hui d'après M.M. Rogers.

No 22 - RR. Gremlii F. var. MaCrocardiacus Sabrs. - Styrie (Sabransky).

№ 123 - R. lasiaxon Borb. et Waisb. - Styrie (K. Fritsch).

M. Fritsch a bien voulu me récolter son $R$. apum pour le Batotheca; ce n'est pas une espèce nouvelle mais très exactement le $R$. lasiaxon B. et Waisb.

No 24 - R. inopreatus M. et L. - France: Valois (Questier).

Plante fertile. C'est exactement le $\mathrm{n}^{\mathrm{e}} 2453$ de Billot (R. infestus, non W. N.)

No 1 - IR. Aavescens M. et L. 1 -France: Valois (Questier).

C'est très exactement le type de Lefèvre!

$\mathrm{Ne}$ - 6 - R. eriostachys M. et Lef, var. acuminatus Sud. - France: Seine-et-Oise (B. de Lesdain). 
c - Hebecaules Sud.

No Az 7 - TR. SCHMIDELYANUS Sud. - France : Vosges (Dr Biau et Lemasson).

No 128 Re. GCHMTDELXANUS Sud., var. BReviglandulosus. - France: Tarn (H. Sudre).

Intermédiaire entre le type et le $R$. Borreri B. Salt.

No $\mathbf{2 0}$ - Re. Bormeri Bell. Salt. - France: Tarn (H. Sudre).

C'est après avoir rencontré de nombreux buissons de cette plante que j'ai acquis la conviction que mon R. pauciglandulosus var. montisparsus, dont je n'avais tout d'abord vu qu'un seul buisson, n’était pas autre chose qu'une variation aprique et robuste du $R$. Borreri. La forme distribuée me paraît identique à celle d'Angleterre (Set of Brit. Ruch. n³8).

No 30 - Re. teretiusculus Kalt. v. ILNGurformis Sprib. - Silésie (Spribille).

No 31 - Re. amictus Müll. ! - France: Marne (Levent).

C'est là le type authenthique de Müller. Commun dans le bois de Mersy, d'après Levent.

No 13:2 - Te. GIEATHOSUG M. et L. Belgique (Gravet).

Me parait identique au type de la forêt de liet\%.

N" 433 - Re GIEATTOSUS M. et L. var. Musectcs Sud. - France: Hte-(xaronne (H sindre).

Voir les caractères dans mes Rub. Europe.

No 1:34 - Re.ellipticifarons Sud. - France: Hte-Garonne (H. Sudre).

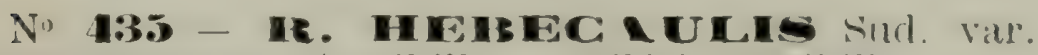

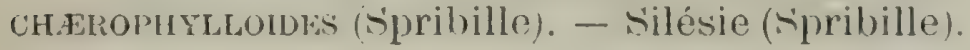


No 436 - TR. HERECANULS Sud. Migr. R. condensatus Müll. var. comptulus Sud. - France: Tarn (H. Sudre).

No 439 - R. salisburgensis F. var, ACUTIFolius Sud. - France : Tarn (H. Sudre).

No 138. - Fe. podophyloides Sud. France: Hte-Garonne (H. Sudre).

La forme distribuée a le turion un peu scabre et se rapproche du $R$. lacteicomus Sud. (gr. du R. micans God.); elle est toutefois moins hétéracanthe.

$$
\begin{aligned}
& \text { C-Radula Focke } \\
& \text { a. - Micantes Sud. }
\end{aligned}
$$

No 439 - Re. TR DUCA Wh. - Saxe (H. Hofmann).

No 10. TR. TR A DUA Wh - France : Sarthe (A. Henry).

Le $R$. Radula Wh. est rare en France. La forme de la Sarthe me paraît se rattacher au type.

No 11 - X R. RADULIFORMiS Sud. ; R. Radula $X$ ulmifolius Sud. - Belgique (du Pré).

Du Pré avait pris cette forme pour un $R$. ulmifolius $X$ vestitus, ainsi qu'en font foi les pétales qu'il avait préparés; mais l'hétéracanthie du rameau et la dentelure des feuilles rappellent le $R$. Radula.

No $142-R$. pustulatus Müll. - France: Hte-Garonne (H. Sudre).

A mal fructifié en 1906 à cause sans doute de l'extrême sécheresse du mois de juin de cette année.

No 143 - R. Bailletii Sud. - France : Hte-Garonne (H. Sudre).

Faciès du $R$. discerptus Müll., mais turion glabre; fleurs d'un rose très vif. 
No 14 - Me. uncinatus P.-J. Müll. - France: Vosges (Dr Biau).

Peu ou point discolore; mais l'été de 1910 ayant èté très pluvieux, le tomentum des feuilles ne s'est pas développé. A peut-être végété dans un endroit couvert?

No 415 - 18. ericetorum Lef. - France : Valois (Questier).

No 446 - Re. ericetorum Lef. - France: Sarthe (A. Henry).

No 14 - Me. aspericaulis $L_{\text {. }}$ et $\mathrm{M}_{\text {. }}$ - France: Valois (Questier).

No $118 \times R$. SEMIASPERICAULIS ; $R$. aspericaulis Xulmifolius. - France: Valois (Questier).

No 419 - Te. pallidiformis Sud. - France : Ariège (H. Sudre).

Est habituellement plus discolore; dans les endroits couverts il devient même tout à fait virescent.

No 450 - Re. papulosus M. et L. - France : Hte-Faronne (H. Sudre).

\section{H. SUDRE.}

Toulouse, aoril 1911. 

$$
\text { . }
$$ 



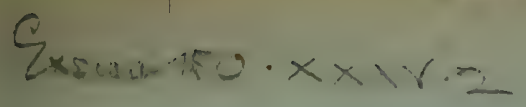

H. Sudre

Batotheca Europarea.

fase. X.

1912. 



\title{
BATOTHECA EUROPЕA
}

PUBLIÉ PAR

\section{HI. SUDRE}

Professeur à l'Ecole normale de Toulouse (France)

12, rue André Délieux

FASCIGULE $X-1912$

mocareron-

Paraît par fascicules annuels de 50 numéros. I,es collaborateurs qui fournissent 5 numéros convenus d'avance et préparés à $20 ̋$ parts, reçoivent un fascicule en échange. Chaque exemplaire doit comprendre, autant que possible, deux rameaux, l'un florifère et l'autre fructifère, plusieur's feuilles caulinaires et quelques pétales préparés à part. Les espèces déja distribućes ne sont admises que si elles diffèrent quelque peu des formes publiées.

\section{Prix du fascicule : 25 fr.}

\section{$+4 \cdot 14 \cdot+\cdots$ \\ COLLECTEURS POUR LE $10^{\circ}$ FASGICULE}

\author{
$\times$ N. Boulay (Nord), no 496. \\ G. Bouvet, à Angers (M. et L.), no 492. \\ Coilliot, au Mans (Sarthe), no 469. \\ L. Corbière, å Cherbourg (Manche), nos 495, 496. \\ K. Friderichsen (Slesvig), no 497. \\ $\times$ G. Genevier (Vendée), no 483. \\ A. Henry, au Mans (Sartle), nos 455, 465, 477, 485, 488, 489. \\ H. Hofmann, à Grossenhain (Saxe), nos 499, 500 . \\ Jaquet (Suisse), no 474 . \\ $\times$ Questier, ¿̀ Thury-en-Talois, nos 454,473, 486, 487, 491, 493. \\ Dr Sabransky, à Scechau (styrie). no 470. \\ H. Sudre, di Toulouse, $11^{n} 451-3$ 456-7, 460-4, 4C6-8, 471-2, 475-6, \\ $479-82,484,498$. \\ Wolley-Dod, at Walton-on-Thames (Angleterre), $\mathbb{1}^{\circ} 459$. \\ Zinsmeister á Wengen (Bavière), nos 458, 478.
}

\section{OBSERVATIONS}

Les collaborateurs sont priés de ne pas ricolter les espuces deja publices dans le Butntheor - ce fascorcule renferme 30 Rubus non distribués dans des exsiccata numerotés. Lo fasc. XI sera consacré aux $R$. rudes et aux $R$. hystrices.

H. SUDRE. 
Sect. IV. - Appendiculati Gen.

C. - Radule Focke.

a. - Micantes Sud.

45 - R. timendus Sud. - Tarn (H. Sudre).

C'est là le type à fleurs blanches, ou à peine rosulées, tel qu'il se rencontre toujours dans le Tarn.

452 - IR. timendus Sud. var. ROSEIFLORUS Sud. - France : Le Laurenti (H. Sudre).

Forme commune dans le Laurenti et le St-Gironnais (Ariège).

$\mathbf{A 5 3}$ - R. FOLIATUS Sud. ; R. TIMENdus $X$ UlmifoLius. - France : Tarn (H. Sudre).

A5A - R. GENEVIERI Bor. - France: Valois (Questier).

C'est la plante publiée par Billot (n 1470) sous le nom de $R$. bracteatus Bor. Me paraît inséparable du type.

455 - R. DISPansus Sud. ; R. GenevieriX ADSCITUS. - France : Sarthe (Henry).

156 - R. brevistachys Sud. - France : Tarn (H. Sudre).

Selon M.M. Rogers, la plante est abondante aux environs de Plymouth, S. Devon. L'auteur du Handbook ajoute : "A very distinct and constant form. " (Handb. p. 72).

La plante distribuée a souffert de la sécheresse; de plus elle avait été coupée l'année précédente et est peu normale; elle est plus grêle et a le tomentum plus ras que d'habitude. Mes spécimens de 1898 ne me paraissent pas différer de ceux de Set of British Rubi no 69 ; ceux de 1904, pris sur les mêmes buissons, s'en éloignent quelque peu, surtout par leurs feuilles 
3-nées, à tomentum presque ras en dessous ; chez quelques fleurs atteintes de pilosisme le calice est étalé.

457-Re. APICULATUSWh. - France: Hte-Garonne (H. Sudre).

Même plante que le n 139 . Comme les spécimens de Timbal étaient maigres, j’ai fait moi-même une centurie de la mème station.

258 - R. APICUI ATUS Wh. - Bavière (Zinsmeister).

Forme un peu grêle, albiflore et peu discolore, pouvant toutefois être rapportée au type.

459 - He. APICULATUS Wh. var. Newboucdil. - Angleterre (Wolley Dod).

C'est exactement la plante de Set of Brit. Rub. $n^{\circ} 66$ !

460 - R. Iauracensis Sud. - France : Lauraguais (H. Sudre).

161 - IR. MICA vs God. - France : Tarn (H. Sudre).

Je l'ai cueilli trop tard, de sorte que les sépales sont presque réfléchis. Sans correspondre exactement à la plante de Nancy, me paraît peu éloigné du type.

462 - TR. MIIANA God. var. schistophilus Sud. - France : H.-Pyrénées (H. Sudre).

Je l'avais cueilli un peu tard, en 1899, et les sépales étaient lâchement réfléchis ; ils étaient manifestement étalés en 1910, ce qui m'a fait rattacher la plante au $R$. micans.

463 - R. MnCANS God. var. estrimabilis Sud. - France : Hte-Garonne (H. Sudre).

S'éloigne beaucoup du type et constitue vraisemblablement une microgène ou une sous-espèce de ce groupe. 
464 - IR. oubrotundus Sud. - France : Hte-Garonne (H. Sudre).

C. dans les régions basses du Tarn et dans la HteGaronne.

A65 - Th. subrotumdus Sud. var. GRANULATifrons Sud. - France : Sarthe (A. Henry).

Rappelle le $R$. granulatus L. et M. par la forme de ses folioles, mais sépales étalés.

$\mathbf{8 6 6 - R}$ - garumnicus Sud. - France : HteGaronne (H. Sudre).

168 - Th. pauciglandulosus Sud. France : Tarn (H. Sudre).

Il ne m’a pas été possible de le cueillir plus tôt ; à la fin les sépales sont lâchement rétléchis, mais ils restent longtemps étalés après l'anthèse.

468 - Re. pauciglandulosus Sud. var. TENEBRIcosus. - France : Tarn (H. Sudre).

Quelques échantillons fructıfères sont aussi trop avancés.

f69 - 16. pauciglandulosus Sud. var. ELLIPTICus. - France : Sarthe (Coilliot).

\&70 - $\times$ MATOUSCHEKII Sabrs. - Styrie (H. Sabransky).

M. Sabranskyi a fait de cette plante un $R$. epipsilus Xmacrostachys, mais son $R$. macrostachys n'est point celui de P.-J. Müller. La dentelur'e fine des feuilles de cet hybride me paraît due au R.holochlorus Sabrs.; qui croît dans les mêmes lieux.

\& 11 - R. subsimihis Sud. - France: HtesPyrénées (H. Sudre).

Presque entièrement virescent en 1910, mais l'été fut très pluvieux. 


\section{$-113-$}

482 - TR. subsimilis Sud. V. OPLOCLADUS. France : Tarn (H. Sudre).

J'ai cueilli cette plante trop tard, de sorte que le calice est devenu entièrement réfléchi ; de plus le buisson ayant été récemment coupé, je n'ai pu l'écolter que des spécimens peu vigoureux montrant mal les caractères de cette variété. J'en distriburrai de plus typiques à la 1re occasion.

A73 - Fi. pulchor M. et L. - France : Valois (Questier).

4. 1 - TR. Gubcamus Müll. var. PILEToneurus Sud. - Suisse (Jaquet).

275 - R. lacteicomus Sud. - France : Tarn (H. Sudre).

J'ai trouvé cette plante dans l'herbier de Progel ; elle croît à Waging (Bavière) (Cf. H. Sudre Rub. Eur.)

\& $\mathbf{6}$ - R. lacteicomus Sud.var. GLOSSOPHYLLOIDES. - France : Hte-Garonne (H. Sudre).

Bien distinct du type et n'en dérivant probablement pas.

b. - Concolores Sud.

A 7 - MR. Obovatifroms Sud. - France: Sarthe (Henry).

Plante un peu discolore, intermédiaire entre $R$.granulatus M. et L. et $R$. timendus Sud.

478 - Tr. traunsteiniensis Kaufm. Bavière (Zinsmeister).

Intermédiaire entre $R$. granulatus M. et L. et $R$. omalus sud. Le $R$. epipsilus Focke f. concolor Progel ! est la même plante.

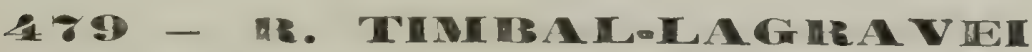
P.-J. Müll. - France : Hte-Garonne (H. Sudre). 
480 - R. virgatus Sud. - France : 'I'arn (H. Sudre).

Plante grêle, précoce, à pollen peu mélangé.

281 - TR. occitanicus Sud. - France : Tarn (H. Sudre).

482 - He.ocitamicns Sud. var. BELLUS. France: Tarn (H. Sudre).

Se rapproche aussi du $R$. subrotundus, mais sépales assez nettement réfléchis.

483 - R. occitamicus Sud, var. CYNOMORUS (Gen.1) - France : Vendée (G. Genevier).

Spécimens authentiques !

\& 8 - R. INCONDITUS Sud. ; R. occitanicUSX ulmifolius. - France : Tarn (H. Sudre).

485 - R. acutipetalus L. et M. - France : Loire-Inférieure (A. Henry).

N'a pa: les pétales aussi nettement aigus que le type de la forêt de Retz, mais il en possède tous les autres caractères.

486 - Rngustifolius M. et L. - France: Valois (Questier).

Exactement conforme au type de Lefèvre!

488 - Fe. retrodentatus M. et L. - Valois (Questier).

488 - apiculatiformis Sud. - France: Sarthe (Henry).

A une tendance à devenir discolore et se rapproche du $R$. apiculalus Wh., mais a l'inflorescence plus armée et le turion plus velu.

489 - R. SEGREENSIS Sud. ; R. APICULATIForMIS $X$ Imbricatus. - France : Maine-et-Loire (A. Henry). 
D'après M. Henry ce Rubus serait commun depuis Segré jusqu'à Châteaubriant (Loire-Inférieure), le long des voies ferrées; il fructifie parfois partiellement.

190 - R. corymbosus P.-J. Müll. - France : Vosges (N. Boulay).

Mème plante que celle des Ronc. vosg.; déterminée par Müller.

491 - R. flexuosus M. et L. 1 - France : Valois (Questier).

C'est le complément de la centurie publiée par Billot $n^{\circ} 2058$.

402 - F. conopectus Gen. - France : Angers (G. Bouvet).

493 - R. truncifolius M. et L. - France : Valois (Questier).

C'est la même plante que le $\mathrm{n}^{0} 1868$ de Billot.

194 - R. trumcifolius M. et L. var. CALLISTEMon Sud. - France : Tarn (H. Sudre).

Plante peu fertile, dont l'origine reste douteuse. Se rapproche des $R$. vestitus et apiculatus, qui manquent dans cette station.

495 - P. truncifolius M. et L. var. THYRSIGERIFormis Sud. - France : Cherbourg (Corbière).

Forme embarrassante. se rapprochant du $R$. apiculatus par ses feuilles un peu discolores, et du $R$. Menkei par ses feuilles en partie 3-nées. Ce nº m'est parvenu sans étiquette, avec le suivant; les 2 formes me paraissent appartenir à la mème variété.

496 - Je. truncifolius MI, et L. v. THYRSIGERIForMis Sud. - France : Cherbourg (L. Corbière).

L'abbé N. Boulay avait distribué cette plante (Ass. $r u b . n^{\circ} 1086$ 1) sous le nom de $R$. uncinatus, mais il n'a 
pas cité ce $n^{\circ}$ dans la Flore de France de Rouy et Camus, ce qui prouve que la plante lui avait paru distincte du $R$. uncinatus Müll.

498 - Re. truncifolius M. et L. var. ACCLINIS Sud. - France (H. Sudre).

498 - R. Drejer.i G. Jens. - Slesvig (K. Friderichsen).

M. K. Friderichsen, en dernier lieu, faisait de cette plante une variété du $R$. horridicaulis P.-J. Müll., ainsi qu'en témoignent les enveloppes des pétales qui accompagnent les spécimens. Mais ce dernierest beaucoup plus hétéracanthe et appartient aux $R$. Hystrices.

499 - R . caliginosus Sud. - France : Puyde-Dồme (H. Sudre).

Plante faible, se rapprochant des $R$. glandulosi.

500 - Ir. PALLIDUS W. et N. - Saxe (H. Hofmann).

\section{H. SUDRE.}

Toulouse, féorier 1912.

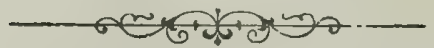



Exsure. $150 \cdot x \times x y \cdot 2$

H. Snidre

Batotheca Euriopaca.

fase. $\overline{X 1}$.

1913 



\section{BATOTHECA EUROPEA}

PUBLIE் PAR

\section{H. SUDRE}

Professeur à l'Ecole normale de Toulouse (France)

12. rue André Délieux

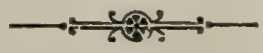

FASCICULE XI- 1913

Parait par fascicules annuels de 50 numéros. Les collaborateurs qui fournissent 5 numéros convenus d'avance et préparés à 25 parts, reçoivent un lascicule en échange; ils payent une cotisation annuelle de 2 fr. pour couvrir les frais d'impression des étiquettes et de port du fascicule qu'ils reçoivent Chaque exemplaire doit comprendre, autant que possible, deux rameaux, l'un florifere et l'autre fructifère, plusieur's feuilles caulinaires et quelques pétales préparés à part. Les espèces déjá distribueés ne sont admises que si elles diffèrent quelque peu des formes publiées.

\section{Prix du fascicule : $\mathbf{2 5}$ fr.}

\section{COLLEGTEURS POUR LE $11^{\circ}$ FASCICULE}

Dr Biau, à Castres (Tarn), nos 516, 518, 539.

Charbonnel, à La Chapelle-Laurent (Cantal), nos 548-550

$\times$ Gravet (Belgique), nos 504, 505 .

Gugler (Bavière), no 525 .

H. Hofmann, à Grossenhain (Saxe), nos 501, 531.

H. Kinscher, à Péterwitz (Silésie), nos 522, 540, 541.

$\times$ P.-J. Müller (Alsace), nos 529.545.

$\times$ Questier, ì Thur r-cll-Valois, nos 502, 503, 507, 527, 532, 538, 546.

D' Sabransky, à Soechau (Sty rie), no 528.

Spribille, prof. à Breslau (Silésie), nos 517, 521, 536.

H. Sudre, à Toulouse, nos 506, 508-515, 519, 520, 526, 530, 534, 535, $537,542,543,544$.

Wolley-Dod, à Walton-on-Thames (Angleterre), no 547.

J.-B. Zinsmeister (Bavière), nos 523, 524, 533.

\section{OBSERVATIONS}

Les collaborateurs sont priés de ne pas récolter les esuèces déja publiées daris le Bratotheca. - Ce fascıcule renferme 30 Rubus non distribués dans les exsiccata numérotés. Le fasc. XII sera consacré aux $R$. Hystrices et aux $R$. glandulosi.

H. SUDRE. 



\section{$-117-$ \\ C - Radulæ Focke}

501 - R. microstachys N. Boul. - Saxe (H. Hof$\operatorname{mann})$.

Croît en société avec les $R$. suberectus, nitidus, villicaulis, candicans, Radula, misniensis, etc., mais ne paraît pas hybride.

502 - R. drymophilus M. et L. - France: Valois (Questier).

503 - R. Chlorocaulon Sud. var. FUsCIFormis. - France : Valois (Questier).

50 - Th. Loh ri Wirtg. var. Foliolatus. Belgique (F. Gravet).

505 - Te. Cohni Wirtg. var. CHLORONEUROIDES Sud. - Belgique (F. Gravet).

506 - Rr. Obscums Kalt. var. PRELATUS Sud. - France: Tarn (H. Sudre).

5oУ - Re. entomodontus P.-J. Müll. France : Oise (Questier).

C'est là le no 2451 de Billot, portant le nom de R. Schleicheri.

508 - R. purpurascens Sud. - France : Tarn (H. Sudre).

509 - R. GRANDIDENTATUS Sud. ; R. PURPUPascens Xulmifolius, - France : Tarn (H. Sudre).

Croît au milieu des parents et est très abondant.

510 - R. obscuriformis Sud. - France : HteGaronne (H. Sudre).

51 - R. obscuriformis Sud. v. pulchrifactus. France : Hte-Garonne (H. Sudre). 
512 - R. DEFECTUS Sud. ; R. OBSCURIForMISX Lloydianus. - France: Hte-Garonne (H. Sudre).

Croît avec le $n^{\circ} 510$ et est stérile.

513 - R. Orraticus Sud. - France : Tarn (H. Sudre).

51 - Re. Oraticus Sud. var. Enostachys Sud. - France : Tarn (H. Sudre).

515 - R. calvifrons Sud. - France : Tarn (H. Sudre).

Grêle, étamines très courtes. Très fertile.

516- TR. MENKEI Wh. - France: Vosges (Dr Biau).

Fructifie incomplètement, sans doute par suite de l'humidité exceptionnelle de l'été en 1910.

517 - R. muricatus B. et Gill. - Silésie (Spribille).

M. Spribille m'a adressé cette plante sous le nom de $R$. twardavicus Sprib, mais elle ne diffère pas du $R$. muricatus de France.

$518-$ R. brachyadenes P.-J. Müll. - France: Vosges (Dr Biau).

$$
\text { D - Rudes Sud. }
$$

519-1R. ON LUS Sud. - France: Tarn (H. Sudre).

C'est la forme vulgaire et typique.

520 - 17. OMLU Sud. var. umbrosus Sud. - France: Pyrénées (H. Sudre).

Cueilli en plein bois, dans un endroit couvert. bille). 
522 - S. Schummelii Wh. - Silésie ( $\mathrm{D}^{\mathrm{r}}$ Kinscher).

523 - R. thelybatus Focke. - Bavière (Zinsmeister).

5 24 - Fe. thellybatus Focke. - Bavière (Zinsmeister).

Ce $n^{0}$ m'a été adressé sous le nom de $R$. epipsilus $\mathrm{X}$ rudis vel epipsilus $\times$ traunsteiniensis par M. Zinsmeister. Bien qu'en partie atteint de pilosisme il fructifie normalement et je ne crois pas que ce soit autre chose qu'une variation du $R$. thelybatus, que le $n^{\circ} 523$ représente plus typique.

\section{5 - R. thelybatus Focke. - Bavière (W. Gugler).}

C'est là le $R$. Gugleri Erdner 1 Ce n'est pas autre chose qu'une variation du $R$. thelybatus $F$. et non un hybride. Les échantillons sont presque tous atteints de pilosisme, ce qui fait que les axes florifères paraissent très hérissés, alors qu'ils sont à peine poilus sur les inflorescences non malades. Bien que ce numéro soit peu normal, je le distribue à cause de son authenticité.

526 - Re. migiduliformis Sud. - France: Tarn (H. Sudre).

527- Re. MELINOXYION M. et Wirtg. - France : Valois (Qnestier).

Il y a quelques feuilles caul. peu normales : habituellement la foliole terminale est à base large et cordée.

$5 \mathbf{5}$ - R. rhodopsis Sabrs. - Styrie (Sabransky).

- Voir la description dans mes Rubi Europo.

529- Re. insolatus Müll. ! - Alsace (P.-J. Müller).

$530-R$. PRAVUS Sud.; R. Superbus XUlmifolius. - France : Tarn (H. Sudre). 
Les premiers rameaux qui ont fleuri ont tous été stériles; d'autres, qui ont fleuri beaucoup plus tard, ont en partie fructifié.

5 - IR. TUDIS Wh. - Saxe (H. Hofmann).

5\$2 - R. RUDIS Wh. - France: Valois (Questier).

$53: 3-R$. RUDINUS Sud.; R. RUdis X BIFrons Sud. - Bavière (Zinsmeister).

53 - R. separatus Sud. - France : Ariège (H. Sudre).

535 - R. GLACELLUS Sud. - France: Tarn (H. Sudre).

536 - Meaberorimus Sud. var. SUBNIGER (Sprib.) - Silésie (Spribille).

Feuilles caul. la plupart 5-nées; fructification partielle.

537 - R. Iuteistylus Sud. - France: Ariège (H: Sudre).

Se rapproche de la var. pubescens Sud. l. c.

$$
\text { E - Hystrices Focke }
$$

538 - TR. FUGO-ATER Wh. var. PHYLLOPHorus (L. et M.) - France : Valois (Questier).

C'est exactement la plante publiée par Questier dans Billot l. c. $\mathrm{N}^{\circ} 3376$.

539 - Re. oñgocladus M. et L. - France : Vosges (Dr Biau).

540 - R. absconditus L. et M. var. sllensanus Kinsch. ! - Silésie (H. Kinscher). 
$5 \mathbf{1}$ - R. SUBROTUNDICAULIS Kinsch.; R. ABSconditus var. silensanus $\times$ Radula Sud. - Silésie (H. Kinscher).

Le tomentum et la dentelure des feuilles rappellent le $R$. Radula Wh.; la coloration de la fleur est celle du $R$. absconditus var. silensanus.

5A2 - R. mutabilis Gen. var. ACAN'THOPHYLLus Sud. - France : Tarn (H. Sudre).

Je distribuerai des rameaux florifères dès qu'il me sera possible d'en récolter.

5\&3 - IR. rubrang P.-J. Müll. - France : Htes-Pyrénées (H. Sudre).

Me paraît exactement la même plante que celle de la Vienne.

5\& \& - F. Pubrans P.-J. Müll. var. ALbeLlus sud. - France : Tarn (H. Sudre).

545 - TR. Lejeunei Wh. var. Atrovirens (P.-J. Müll.!) - Alsace (Müller).

Turion distinctement velu; foliole caulinaire terminale ovale ou largement rhombée, inflorescence hérissée, moins lâche que dans le $R$. Lejeune $i$ Whe. J'en ai vu des spécimens de Climbach (butte calcaire), de Bobenthal et du rameau latéral du vallon de la Lauterbach (grès vosgien), près de Wissembourg. Ces différents échantillons ne m'ont pas paru différer sensiblement les uns des autres. Il est possible que les pétales distribués proviennent de Climbach. Le pollen est très peu mélangé sur les exemplaires où j'ai pu l'observer, et il y a lieu de croire que cette Ronce est une forme pure.

56- It formidabilis L. et M. - France : Valois (Questier).

C'est la plante distribuée par Questier dans les exsiccata de Billot $n^{\circ} 970$, sous le nom de $R$. Lejeunei (non Wh.)

5\&8 - Te formidabilis L. et M. - Angleterre: - Surrey (Wolley-Dod). 
5\&8 - IR. drepanophorus Sud. - France: Cantal (Charbonnel).

549 - Fe. drepanophorus Sud. - France: Cantal (Charbonnel).

Forme aprique, plus ou moins atteinte de pilosisme, ayant une tendance à devenir un peu discolore.

550 - R. DREPANOPHOROMORPHUS Sud.; $R$. drepanophorus $\times$ Lloydianus. - France : Cantal (Charbonnel).

\section{H. SUDRE.}

Toulouse, Janvier 1913.

Alb1.-Impr. A. Nouguits, rue de 1'Hôtel-de-Ville, 33 


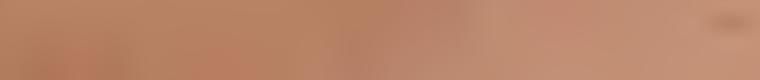
:
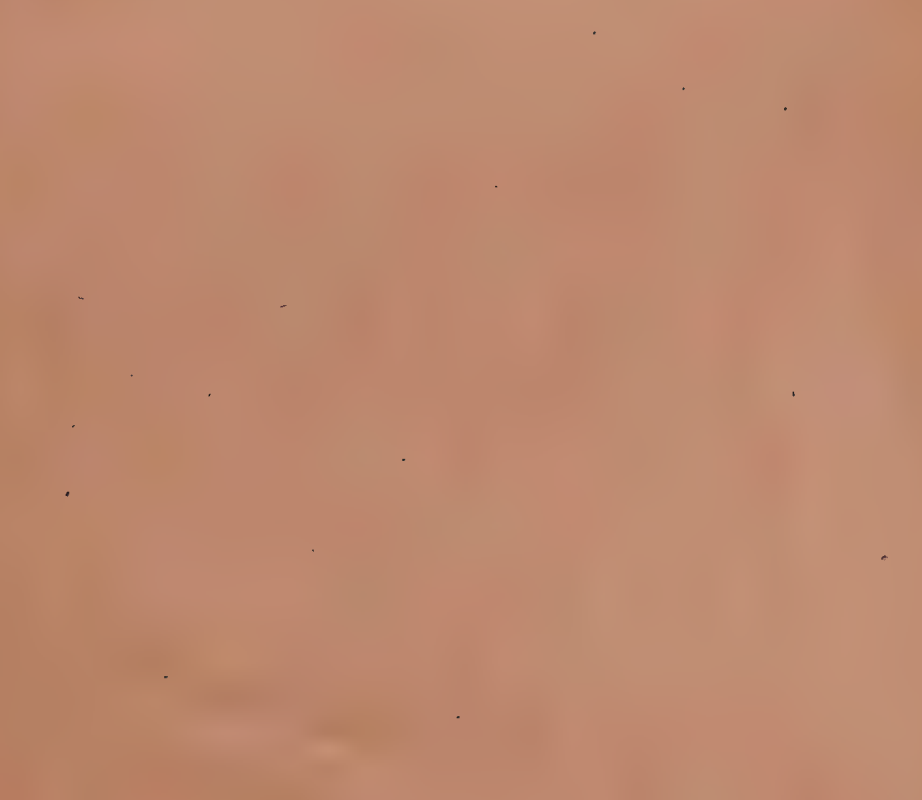

, 

Crisce. $150 \cdot x \times x v \cdot 2^{\text {JUL. } 1914}$

H. Sndre

Batotheca Europacea.

fase. XII.

1914 



\title{
BATOTHECA EUROPEA
}

PUBLIÉ PAR

\author{
H. SUDRE \\ Professeur à l'Ecole Normale de Toulouse (France) \\ 12, rue André Délieux
}

FASCICULE XII - 1914

Parait par fascicules annuels de 50 numéros. I.es collaborateurs qui fournissent 5 numéros convenus d'avance et préparés à 20 parts, recoivent un fascicule en échange; ils payent une cotisation annuelle de 2 fr. pour couvrir les frais d'impression des étiquettes et de port du fascicule qui leur est attribué. Chaque exemplaire doit comprendre, autant que possible, deux rameaux, l'un florifère et l'autre fructifère, plusieurs feuilles caulinaires et quelques pétales préparés à part. Les espèces déjà distribuées ne sont admises que si elles diffèrent quelque peu des formes publiées.

\section{Prix du fascicule : 25 fr.}

\section{COLLEGTEURS POUR LE 12 ${ }^{\circ}$ FASGIGULE}

Dr Biau, à Castres (Tarn), no 568.

$D^{r}$ Bouly de Lesdain, Dunkerque (Nord), Nos 557, 567, 598, 600.

J.-B. Charbonnel, à La Chapelle-Laurent (Cantal), nº 592.

Duffort, Masseube (Gers), No 552.

Dr o. Focke, Bremen (Allemagne), No 597.

$\times$ Gravet (Belgique), no 551 .

A. Henry, Le Mans (Sarthe), No 570.

H. Hofmann, à Grossenhain (Saxe), nos $563,579,588$.

Jourdes, Verneuil (Eure), No 556.

Dr Kinscher. Steinach (Thuringe), nos 555, 561, 565, 571, 572.

s. Kupcok, Bakabanya (Hongrie), No 581 .

$\times$ Questier, à Thury-en-Valois, nos 552. 554, 520, 585.

Dr Sabransky, à Sœehau (Sty rie), no 577, 582, 583.

Spribille, prof. à Breslau (Silésie), nos $564,599$.

H. Sudre, à Toulouse, nos $558,559,560,566,569,573-6,578,584,586$, $587,589-91,593-6$.

Zinsmeister, Wengen (Bavière), no 553.

\section{OBSERVATIONS}

Les collaborateurs sont priés de ne pas récolter les espèces déjà publiées dans le Batothesa. - Ce fascicule renferme 35 Rubus non distribués dans des exsiccata numérotés. Le fasc. XIII sera consacré aux $R$. glundulosi.

H. SUDRE. 
Sect. IV. - Appendiculati Gen.

E - Hystrices Focke

551 - F. coronatus N. Boul. - Belgique (Gravet).

552 - F. HYSTREX Wh. var. VELATUS (Lef.) - France: Valois (Questier).

D'après Genevier la couleur des organes floraux serait : pétales roses, étamines et styles pâles.

553 - R. bavaricus F'ocke. - Bavière (Zinsmeister).

554 - R. pinulifer M. et L. - France : Valois (Questier).

S'éloigne beaucoup du type $R$. Koehler $i \mathrm{~W}$. et ne saurait lui être rattaché comme simple variété.

555 - R. spinulifer M. et L. - Silésie (H. Kinscher).

Le $R$. subconicus Kinsch., que représente ce No, me paraît la même plante que le No 554 .

556 - R. asperidens Sud. - France : Eure-et-Loir (Jourdes).

Quelques échantillons sont atteints de pilosisme et montrent mal les caractères de l'espèce ; ordinairement les axes florifères sont plus armés.

55 - R. asperidens Sud. - France: Seine-et-Oise (B. de Lesdain).

Cueilli un peu trop tôt; sur quelques spécimens les sépales s'étalent manifestement.

558 - R. asperidens Sud. var. HISPIDUllCaulis. - France : Seine-et-Oise (H. Sudre).

A fleuri pour la $2^{\mathrm{e}}$ fois en 1910. - Ne diffère en rien de la plante sauvage de la forêt de Rambouillet, d'où il m'a été envoyé par Mlle Bélèze. 
559 - Re. Ieptocercus Sud. - France : Ariège (H. Sudre).

Calice étalé ; folioles terminales longuement acuminées.

560 - R. SEPARATIFORMIS Sud. - R. LEPTOcercus Xseparatus. - France : Ariêge (H. Sudre).

561 - IR. Feutoni Merc. var. Pygmeopsis Sud. - Allemagne : Silésie (H. Kinscher).

562 - He. He outeri Merc. var. PygMeofsis. France: Gers (Duffort).

Plus robuste que le No 561, mais mêmes caractères généraux.

563 - Re. apricus Wimmer. - Allemagne: Saxe (H. Hofmann).

D'après M. Hofmann. l'espèce, commune en Silésie, est très rare dans la Saxe.

56 - R. subpygmæopsis Sprib. - Silésie (Spribille).

$565-$ R. pygmæus Wh. - Silésie (H. Kinscher).

566 - Te. saxicolus P.-J. Müll. - France (H. Sudre).

Plante née en 1898 et ayant fleuri pour la $3^{\mathrm{e}}$ fois en 1901, époque à laquelle j'ai quitté Albi et n'ai pu poursuivre mes observations sur cette forme. Elle est encore jeune et peu normale, avec des turions arrondis et des feuilles en partie 3-nées ; mais la plantemère de Cauterets me paraît pouvoir être rapportée au type $R$. saxicolus Müll. J'ai obtenu, du même semis, une forme bien différente, stérile et à étamines longues.

56 - Te. saxicolus P.-J. Müll. var. PARlsiensis Sud. - France: Seine-et-Oise (B. de Lesdain). 
568 - R. horridicaulis P.-J. Müll. - France : Vosges (A. Biau).

Feuilles caulinaires la plupart peu normales. On doit toujours les prélever sur des turions bien développés et vers le milieu de ceux-ci.

569 - Fe. rotundellus Sud. - France : Cantal (H. Sudre).

57O - R. Totundellus Sud. var. PSEUDOGLAUcellus. - France : Loire-Inférieure (Henry).

581 - Re. rotundellus Sud. v. PLATYDACTYLus (Kinscher). - Silésie (H. Kinscher).

$5 \boldsymbol{7} \mathbf{2}-\mathrm{R}$. polyoplon B. et Mot. v. RHAPHIDORACHIS (Kinscher). - Silésie (H. Kinscher).

57 - R. impolitus Sud. - France : Gers (H. Sudre).

F - Glandulosi P.-J. Müll.

58 4- TR. FURVUS Sud. var. FAllax. France : Tarn (H. Sudre).

585 - RR. PUEPURATUS Sud. France : Tarn (H. Sudre).

La plante distribuée est intermédiaire entre le type et la var. tenerrimus Sud. $l$. $c$.

576 - R. prædatus Schmid. - France : Tarn (H. Sudre).

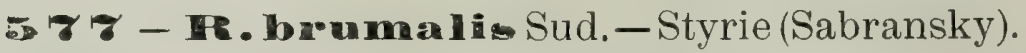

588 - R. brachyandroides Sud. - France : Ariège (H. Sudre).

579 - TE. SCARER Wh. - Saxe (H. Hofmann).

Calice lâchement réfléchi. 
580 - Re. TERETICA ULIS P.-J. Müll. var. Microdontus (M. et L.) - France : Forêt de Retz (Questier).

581 - TR. TERETICAULIS P.-J. Müll. var. Lipo'trichus (S. Kpk). - Hongrie (S. Kupcok).

Fertile et non hybride de $\boldsymbol{R}$. thyrsanthus F.; a le turion glabrescent et se rapproche aussi du $R$. finitimus Sud.

582 - R. TET ETICAUTIS P.-J.Müll. var. Rubristyliformis Sud. - Styrie (Sabransky).

Se rapproche du $R$. finitimus var. rubristylus Sud., mais ses turions très pubescents me le font rattacher au $R$. tereticaulis P.-J. Müll. Pétales larges, glandes très courtes.

583 - R. FRITSCHII Sabrs. ; R. TERETICAULisX DURIMontanus Sud. - Styrie (Saloransky).

584 - R. fragariiflorus P. J. Müll. var. SERPENTIFormis Sud. - France : Tarn (H. Sudre).

Se rapproche du $R$. chlorostachys Müll. du gr. du $R$. serpens Wh., mais a les glandes courtes du $R$. tereticaulis Müll.

585 - He. derosifolius Sud. - France : Valois (Questier).

586 - R. derasifoliug Sud. - France : Massif d'Arbas (H. Sudre).

589 - R. cuntiglandulosus Sud. - France: Tarn (H. Sudre).

Bien que les carpelles soient glabres, la plante peut être rapportée au type, qui les a velus.

588 - Le cumtiglandulosus Sud. - Saxe (H. Hofmann).

589 - IE cuntiglanduloens Sud. var. PRoTensus Sud. - France: Tarn (H. Sudre). 
590 - R. subalpinus Sud. - France : Htes-Pyrénées (H. Sudre).

591 - R. argutipilus Sud. - France : Tarn (H. Sudre).

592 - F. argutipilus Sud. var. ANAMPHIESTus (G. Br.) - France : Cantal (Charbonnel).

Turion pruineux, glandes pâles. Se rapproche du $R$. serpens Wh.

593 - Fe. argutipilus Sud. var. ARBASCENSIS. - France : Hte-Garonne (H. Sudre).

Se rapproche du $R$. purpuratus Sud., qui croît dans le même massif.

594 - R. finitimus Sud. - France: Tarn (H. Sudre).

Plus grêle que le type primitif de Cauterets.

595 - R. finitimus Sud. var. Megathyrsus. France : 'Tarn (H. Sudre).

Inflorescence vaste, aphylle, poilue ; folioles larges ; glandes pâles.

$\mathbf{5 9 6}$ - R. finitimus Sud. var. simulans. - France: Tarn (H. Sudre).

Diffère du $R$. curtiglandulosus Sud. par ses turions glabres, ses étamines courtes; du $R$. argutipilus Sud. par ses étamines moins longues.

598 - IR. SCHLEETHERI Wh. - Allemagne (Focke).

598 - R. SCHILEICHEIRI Wh, var. CONJugatus Sud. - France : Nord (B. de Lesdain).

599 - IR. SCHILECHETE Wh. var. Mitis Wimm. et Grab. - Silésie (Spribille). 
600 - IR. SCHILETCHERI Wh. var. SEquanensis Sud. - France: Seine (B. de Lesdain).

Croît avec le $R$. saxicolus var. parisiensis. Se rapproche des $R$. hirlus W. et K. et humifusus Wh.

\section{H. SUDRE.}

Toulouse, avril 1914.

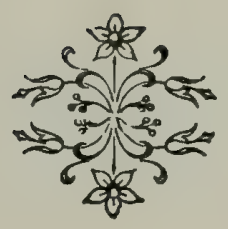


JUL. 1914 


$k+\cdots=0$

$5 \pi=196$

Xax-

He. Sudre.

Batotheca Europaiea.

Yase.
I9III. 



\title{
BATOTHECA EUROP瓜A
}

PÜBLIÉ PAR

\author{
H. SUDRE \\ Professeur à l'Ecole Normale de Toulouse (Franco) \\ 3, rue André Délieux
}

FASCICULE XIII - 1915

Parait par fascicules annuels de 50 numéros. I.es collaborateurs qui fournissent 5 numéros convenus d'avance et préparés à 25 parts recoivent un fascicule en échange; ils payent une cotisation annuelle de $2 \mathrm{fr}$. pour couvrir les frais i impression des étiquettes et de port du fascicule qui leur est attribué. Shaque exemplaire doit comprendre, autant que possible, deux rameaux, l'un florifere et l'autre fructi. f're, plusicurs feuilles caulinaires et quelques pétales priparés i jart. Les espèces déjà distribuées ne sont admises que si elles diffèrent quelque peu des formes publiées.

\section{Prix du fascicule: 25 ir.}

\section{COLLEGTEURS POUR LE $13^{\circ}$ FASGIGULE}

Dr Biau, à Castres (Tarn), no 636 .

$D^{r}$ Bouly de Lesdain, à Dunkerque -(Nord), no 603.

$\times D^{r}$ Gillot (Saòne-et-Loire), n 604 ,

$\times$ F. Gravet (Belgique), nos 631, 639 .

A. Henry, Le Mans (Sarthe), nos 628, 645, 648.

H. Hofmann, à Grossenhain (Saxe), nos 608, 615 .

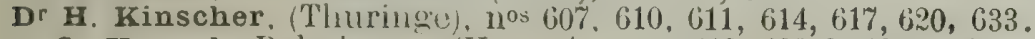

X S. Kupcok, Bakahany (Hongrie), nus 619, 622-3, 626, 642-3.

$\times$ Levent (Marne), no 640.

$\times \mathbf{P} \cdot$-J Müller (Alsace), nos 624-5, 629.

$\times$ Pierrat (Vosges, uo 630 .

$x$ Questier (Valois), nos $634,644.650$.

Dr H. Sabransky, (Styrie), nos $612,618,621,646$.

Spribille, Breslau, nos $601,616,627,638$.

H. Sudre, Toulouse, $1^{0 \mathrm{~s}} 602,604-6,635,637,641,647.649$.

J.-B. Zinsmeister, (Souabe), nos 613, 632.

\section{OBSERVATIONS}

Les collabolateurs sont priés de ne pas récoltel les especes déja publices dans le Batotheea. Cie fascicule renferme 36 Rubus non distribués dans des exsiceata numérotés. Le fasc. XIV sera consacré aux $R$. Glundulosi et aux $R$. Trioiales.

H. SUDRE. 


\section{Appendiculati Gen. \\ F - Glandulosi P.-J. Müll.}

601 - R. INFESTIFRONS Sud. et Sprib. - Silésie (Pribille).

Semble bien dériver du $R$. silesiacus Wh.; l'autre parent est douteux.

Rappelle un peu le $R$. infestus Wh. D'après M. Spribille, ce serait là le $R$. thyrsiflorus Wimm. (non Wh.).

$60=-$ R. dissectifolius Sud. - France : Tarn (H. Sudre).

60: - R. chloroxylon Sud. - France : Nord (B. de Lesdain).

$604-\mathrm{R}$. conterminus Sud. var. piletofructus. France : Hte-Garonne (H. Sudre).

605 - R. conterminus Sud. var. saxorum. France: massif d'Arbas (H. Sudre).

606 - R. laceratus Müll. var. incequabilifrons Sud. - France: Tarn (H. Sudre).

6O - R. humifusus Wh. -. Silésie (Kinscher).

Ne paraît pas différer sensiblement du type ; toutefois fructification incomplète, ce qui peut être dù à la station.

608 - R. humifusus Wh. var. brachyslachys (Müll.). - Saxe (H. Hofmann).

609 - R. humifusus Wh. var. humifusiformis Sud.-France: Morvan (Gillot).

$610-\mathrm{R}$. BICOLORIFULIUS Kinsch.; $R$. humifusus $\times$ Radula Sud. - Silésie (Kinscher).

611 - R. irrufatus P.-J. Müll. - Silésie (Kinscher). 
61 $\geq-\mathrm{R}$. inæquabilis Sud. var. stylosus (Sabrs. 1). - Styrie (H. Sabransky).

M. Sabransky a rapproché sa plante du $R$. thyrsifloric.s Wh., mais ses glandes longues, son inflorescence aciculée, me la font ranger dans les $R$. glandulosi.

613 - R. PIVUU IS M. et Wirtg. Souabe (Zinsmeister).

Pédoncules plus étalés "et inflorescence moins aciculée que dans le type, sans qu'il y ait lieu d'y voir une variété notable.

$614-1$. RIVUC are M. et Wirtg. (Kinscher).

11 y a quelques feuilles caulinaires (prises sans doute vers la base du turion) qui sont largement ovales et qui rappellent un peu celles du $R$. setiger L. et M.

(15 - R. lusaticus Rost. - Saxe (Hofmann).

66 - R. lusaticus Rost. var. heidewilxensis (Sprib.). - Silésie (Spribille).

$61-\mathrm{R}$. incultus Wirtg. var. subincultus Sud.Silésie (Kinscher).

$618-R$. PERSETOSUS Sabrs. ; R. spinosulus $\times$ bifrons Sud. - Styrie (Sabransky).

Aculéation du $R$. spinosulus; tomentum du $R$. bifrons. Peu ou point fertile.

(1) 9 - R. VIETUS Kupcok ; R. spinosulus $X$ Lloydianus sud. - Hongrie (S. Kupcok).

(5)0 - R. horridulus Mïll. var. rigidatus Sud. Silésie (Kinscher).

Me parait bien être la même plante que le $n^{\circ} 159$ de Gremli (IIerb. norm.) portant le nom de $R$. rigidatus (irl.

6 $21-R$. horridulus Müll, var. canifolius (Hayek). - Styrie (H. Sabransky). 
Me paraît simple var. du $R$. horridulus et non hybride. - J'ai dû éliminer de la certurie des feuilles très discolores qui appartenaicnt à une forme différente.

B: $\mathbf{2}-\mathbf{R}$. horridulus Müll. |var. Degenianus (Kupcok). - Hongrie (S. Kupcok).

Très rapproché du $R$. spinosulus Sud.; mais étamines courtes. Certains rameaux ont des glandes pourpres et tendent vers le $R$. hirtus W. K. (minutiflorus).

623 - R. VRANYI Kupcok; $R$. leptobelus $\times b i$ frons Sud. - Hongrie (S. Kupcok).

Peu ou point fertile.

621 - T. SERPENA Wh. var. elegans (Müll. !). — Alsace (Müller).

6:5 - R. SERPRNS Wh. var. calliphyllus (Müll.!). - Alsace (Müller).

626 - R. KUPCOKIANUS Borbas $!$; $R$. serpens $\times$ Lloydianus Sud. - Hongrie (S. Kupcok).

Stérile. L’influence des 2 parents est manifeste.

628 - IR. napophiloides Sud. - Silésie (Spribille).

6:8 - R. mapophiloides Sud. - France: Sarthe (A. Henry).

Les $n^{\text {us }} 627,628$, sans être identiques, peuvent ètre rapprochés du type. 628 a parfois des glandes un peu rougeâtres et tend vers $R$. hirtus W. K. ler).

A29 - R. corylinus P.-J. Müll.! - Alsace (Mül-

Spécimens authentiques.

4:30 - R. corylinus P.-J Müll. var. reclinatispinus Sud. - France: Vosges (Pierrat). 
631 - IR. Inccidifolius Müll. - Belgique (F. Gravet).

Peu éloigné du type vosgien.

632 - R. Aaccidifolius Müll. var. membrınaceus Sud. - Bavière (Zinsmeister).

Se rapproche de $R$. vepallidus Sud. ; toutefois dentelure plus fine.

6:53- R. Aaccidi folius Müll. var. Kinscheri (Sprib.) - Silésie (Kinscher̃).

Feuillage ample du $R$. flaccidifolius, mais dentelure grossière, inégale, rapprochant la plante du $R$. obrosus Müll.

63 $4-R$. analogus M. et L. - France : forêt de Retz (Questier).

Même plante que le No $205 \%$ de Billot (R. Guentheri).

655 - F. vepallidus Sud. - France : Tarn (H. Sudre).

636 - Le. chloxostachys Müll. - France : Vosges (Biau).

637 - 16. crinitus Sud. - France : Tarn (Sudre).

De la mème station que le $\mathrm{n}^{\circ} 43$ du Batotheca.

A38 - R. Ioptadonos Sud. var. lividus G. Br. - Saxe (Spribille).

639 - R. LEPTADENOIDES sud.; R. leptadenes Xsilvaticus ? - Belgique (Gravet).

Le regretté F. Gravet m'avait adressé cette plante sous le nom de $R$. podophyllus (Müll.); elle est vraiseinblablement hybride, mais lorigine en reste douteuse. Est stérile.

620 - Re. oreades M. et Wirtg. var. brevispiculatus sud. - France : Marne (Levent). 
Très rapproché du $R$. serpens var. calliphyllus (Müll.), toutefois feuilles caul. la plupart 3-nées, aiguillons plus courts, turion et axe florifère moins velus, étamines plus courtes.

(1) 1 - R. offensus Müll. var. gracilescens Sud. France : Tarn (H. Sudre).

Fleurs blanches; étamines dépassant les styles verdâtres. Remarquable par sa station très basse. - Très grêle ; dentelure très fine.

Gs2 - Fo. Guntheri Wh. var. Lengyellii (Kupcolk). - Hongrie (Kupcolk).

Fructifie normalement et ne paraît pas hybride.

643 - R. RIPENSIS Kupcok; R. Guentheri $X$ rivularis Sud. - (Hongrie (S. Kupcok).

Certains rameaux rappellent le $R$. rivularis et d'autres le $R$. Guentheri.

64 - 1 - nignicatus M. et Lef. - France : Valois (Questier).

C'est là le $R$. hirtus de Billot (n²056 p. p.).

645 - R. nignicatus M. et L. -- France: Sarthe (Henry).

616-R. pectinatus Sud. et Gr. v. chlorosericeus Sabrs. - Styrie (Sabransky).

$61-\mathrm{R}$. pectinatus Sud. et Gr. v. adenoneurus Sud. - France : Tarn (H. Sudre).

Récolté aux Vidals, le long du chemin de Montalet.

6 - 8 - R. pectinatus Sud. et Gr. var. Henryanus Sud. - France : Sarthe (Henry).

649 - M. Kaltembachii Metsch var. fore¿iacus Sud. - France : Puy-de-Dôme (H. Sudre).

650 - R. rubiginosus Müll. var. trichacanthus (Müll.). - France : Oise (Questier). 


\section{$-137-$}

Je distribue le complément de la centurie de Billot no 2056 (р. p.) qui représente le $R$. trichacanthus Müll.

\section{H. SUDRE.}

Toulouse, mai 1915.

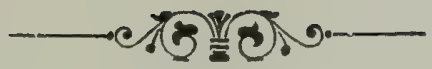


6. 13. 




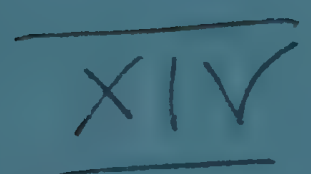





\section{BATOTHECA EUROP在A}

PUBLIÉ PAR

H. SUDRE

Professeur à l'Ecole Normale de Toulouse (France)

3, rue André Délieux

FASCICULE XIV - 1916

Paraît par fascicules annuels de 50 numéros. Les collaborateurs qui fournissent כૅ numéros convenus d'avance et préparés à 20 parts reçoivent un fascicule en échange; ils payent une cotisation annuelle de 2 fr. pour couvrir les frais d'impression des étiquettes et le port du fascicule qu'ils reçoivent. Chaque exemplaire doit comprendre, autant que possible, deux rameaux, l'un florifere et l'autre fructifère, plusieurs feuilles caulinaires et quelques pétales préparés à part. Les espèces déjà distribuées ne sont admises que si elles diffèrent quelque peu des formes publiées.

\section{Prix du fascicule : $\mathbf{2 5}$ fr.}

\section{COLLECTEURS POUR LE $14^{\circ}$ FASCICULE}

C. Bicknell a Bordighera (Italie), nº 683 ,

Dr Biau, à Castres (Tarn), no 667 .

$\times$ Billot (Alsace), no 689 .

J.-B. Charbonnel. La Chapelle-Laurent (Cantal), nos 675, 684, 687. $\times$ Chaboisseau (Vienne), $n^{6} 681$.

Charrier (Vendée), no 676 .

$\times$ Coilliot, Le Mans (Sarthe), n ${ }^{\circ} 688$.

Duffort, à Masseube (Gers), no 680 .

A. Henry, Le Mans (Sarthe), no 673.

H. Eolmann, à Grossenhain (Saxe), nos 662, 665, 66\%.

Holzfuss, à Stettin (Poméranie), nus 661, 663, 666, 670, 671, 692, 696.

Dr Kinscher, Steinach (Thuringe), no 668.

$\times$ S. Kupcok (Hongrie), no 652 .

$\times$ Levent (Marne), nos 682, 686.

$\times$ P.-J. Müller (Alsace), nos 653, 672, 685, 690, 695, 697, 699.

$\times$ Questier (Valois), nos $654,669,678,693$.

Dr H. Sabransky (Sty rie), no 655 .

Spribille, Breslau, (Silésie), nº 691.

H. Sudre, à Toulouse, nos $654,656-660,674,679,694,698,700$.

J.-B. Zinsmeister (Bavière), nº 664 .

\section{OBSERVATIONS}

Les collaborateurs sont priés de ne pas récolter les espèces déjà publiées dans le Batotheca. Ce fascicule renferme 26 Rubus non distribués dans des exsiccata numérotés.

H. SUDRE. 
Sect. IV - Appendiculati Gen.

$$
\text { F-GLandulosı P.-J. Müll. }
$$

651 - R. ACICULARIS L. et M. ; R. rubig. v. trichacanthus $入$ horrens Sud. - France: Valois (Questier).

Paraît assez répandu du côté de Lévignien, mais toujours presque stérile.

65: - R. CUNICULICOLUS Kpk. ; $R$. rubiginosus $\times$ mutabilis ? - Hongrie (Kupcok).

Les glandes pourpres montrent l'influence d'une forme du groupe hirtus, apparemment le R. rubiginosis; l'autre parent est douteux.

653 - R. minutiflorus Müll. ! - Alsace (Müller). Spécimens authentiques!

65 - R. minutiflorus Müll. var. horridifactus Sud. - France : Tarn (H. Sudre).

655 - R. HOPLOPHORUS Sabrs. ; R. minutiflorus var. Xholochorus. - Styrie (Sabransky).

Glandes et aculéation du R. minutiflorus, mais dentelure, pilosité et forme des feuilles rappelant le $R$. holochlorus. Peu fertile.

656 - R. trachyadenes Sud. var. glabrescens. France : Tarn (H. Sudre).

Dentelure moins grossière que d'habitude. Se rapproche un peu du $R$. tenuidentatus var. jaclabundus.

$\underset{\text { dre). }}{\mathbf{6 5}} \mathbf{7}-\mathbf{R}$. declivis Sud. - France : Tarn (H. Su-

Floraison très précoce; station très basse. Très fertile.

658 - R. VIDUATUS Sud.; R. declivis $\times$ lacertosus.

- France : Tarn (H. Sudre).

En société avec les parents et tout à fait stérile. 
Sect. V - Triviales P.-J. Müll.

659 - H. CAECYUS L. var. mitissimus (Rip.) - France : Tarn (H. Sudre).

660 - R. SERRULATUS Lindg. : $R$. nitidus Xcoesius. - France : Tarn (H. Sudre).

Peu ou point fertile. Le $R$. nitidus n'est pas rare dans la région.

661 - R. SCABROSIFORMIS Sud.; $R$. integribasis $\times$ coesius. - Allemagne : Poméranie (Holzfuss).

Forme très rapprochée du $R$. integribasis Müll.

$662-\mathrm{R}$. GOTHICUS Fr. et Gel. ; $R$. gratus $X$ coesius Sud. - Saxe (Hofmann).

663 - R. SEMISPRENGELII Sud. ; $R$. Sprengelii casius. - Poméranie (Holzfuss).

Fleurs et principaux caractères du $R$. Sprengelii Vh., mais pétales et folioles larges. Fructifie partiellement.

66 - R. ZINSMEISTERI Erdner ! ; R. rhombifolius $\times$ cosius Sud. - Bavière (Zinsmeister).

Considéré par l'auteur comme un $R$. coesius $\times$ epipsilus, mais ne rappelle nullement ce dernier.

66-5 - R. WARNSTORFIl Focke; $R$. silesiacus $X$ coesius Sud. - Saxe (H. Hofmann).

Le $R$. silesiacus Wh. se rencontre dans la même localité, d'après M. Hofmann.

$666-$ R. SEMIPYRAMIDALIS Sud. ; R. pyramidalis Xccesius. - Poméranie (E. Holzfuss).

La pilosité de la face inférieure des feuilles ne peut provenir que du $R$. pyramidalis Kalt., qni est fréquent dans la région.

66 - R. BALFOURIANUS Blox. - France : Vosges (Biau).

Hybride fixé dontl'origine reste douteuse. 
668 - R. BALFOURIANUS Blox. ; $R$. ciliolentus Kinscher ! - Silésie (Kinscher).

M. Kinscher a considéré cette plante comme un R. casius $\times$ Balfourianus. Il ne me parait pas qu'il y ait lieu de la séparer du $n^{\circ} 66 \%$.

669 - R. UMBELLIFORMIS M. et L. !; $R$. macrophyllus $\times$ cosius Sud. - France: Valois (Questier).

Plus rapproché du $R$. macrophyllus que du $R$. ccesius; très peu fertile.

6-8 - R. SEDINENSIS Holzfuss; $R$. nemorensis $X$ coesius Sud. Poméranie (Holzfuss).

Rappelle beaucoup le $R$. nemorensis L. et M., que M. Holzfuss m'a adressé des environs de Stettin.

6r 1 - R. PRUINOSUS Arrh. ; R. casius Xvillicaulis Sud. - Poméranie (Holzfuss).

Forme aprique, un peu discolore, se rapprochant du R. Wahlbergii Arrh.

6 子 $\approx-R$. CARNEISTYLUS Müll.!; R. cosius $\times$ incarnatus Sud. - Alsace (Müller).

Très rapproché des nos 667,668 , mais inflorescence non glanduleuse, turion presque grabre. Pourrait je crois dériver du $R$. incarnatus, qui croît dans les mêmes lieux.

67: - R. UPLOTHYRSOIDES Sud.; $R$. oplothyrsus $\times$ cossius. - France : Sarthe (Henry).

Croît dans le voisinage du $R$. oplothyrsus et fructifie partiellement.

6 子 4 - R. HEMILASIOTHYRSUS Sud.; $R$. lasiothyrsus $\times$ ccesius. - France : Hte-Garonne (H. Sudre).

Rappelle un peu les hybrides de coesius et dé tomen losus, toutefois turion pubescent, dentelure plus fine. Le $R$. lasiolhyrsus est CC. dans cette région des Pyrénées.

675 - R. SEMIALBIFLORUS Sud. ; $R$. albiflorus $\times$ ccesius. - France : Cantal (Charbonnel). 
6r6 - R. IMBRICATIFORMIS Sud. ; R. imbricatus $\times$ cesius. - France : Vendée (Charrier).

Forme aprique et peu normale, dérivant du $R$. imbricatus var. virescens.

6z $\boldsymbol{\gamma}$ - R. IMBIRICATIFORMIS Sud. var. semisaxo. nicus Sud.; $R$. imbricatus var. saxonicus Hfm. Xcesius - Saxe (Hofmann).

Très rapproché de la var. saxonicus ( $R$. saxonicus Hfn). Le $R$. Wahlbergii dérive du $R$. villicaulis Kœhl,

68 8 - R. VALESIENSIS Sud. ; R. cardiophyllus X cesius. - France : Valois (Questier).

679- R. MARTRINII Sud. - France : Tarn (H. Sudre).

Probablement hybride fixé de $R$. casius et de R. ulmifolius.

Fertile et très répandu.

680 - R. AMPLIFOLIATUS Sud.; R. ulmifolius $X$ casius. - France : Gers (Duffort).

Stérile.

681 - R. AMPLIFOLIATUS Sud. var. thamnocharis (Müll. !)

Spécimens authentiques réunis par Chaboisseau pour les exsiccata de Müller.

B8: - R. AMPLIFOLIATUS Sud. var. acanthophorus (Müll.) - France : Marne (Levent.

683 - R. ASSURGENS B. et Bv. f. Provostii Gen.; R. coesius $\times$ ulmifolius. - Italie (C. Bicknell).

(68 - R. ASSURGENS B. et Bv. var. polyadenes Sud. - France : Hte-Loire (Charbonnel).

Provient de quelque forme très aculéolée de $R$.ulmifolius.

685 - R. AMBIGUUS Müll.! R. GodroniiXcasius. - Alsace (Müller). 
Quelques échantillons étaient sans étiquette, mais tous proviennent apparemment de la même localité.

686 - R. DISCOIDEUS Müll. 1 ; $R$. WinteriX casius Sud. - France : Marne (Levent).

687 - R. DURICORIUS Gen. $p . p . ; R$. casius $\times$ Winteri Sud. - France : Cantal (Charbonnel).

Ronce grêle, rappelant le $R$. Winteri par la forme des folioles et la dentelure des feuilles.

689 - R. MACROPETALUS M. et L. ; $\boldsymbol{R}$. propinquus $\times$ ceesius Sud. - France : Sarthe (Coilliot).

689 - R. MOUGEOTII Billot!; R. casius $\times b i-$ frons Sud. - Alsace (Billot).

Echantillons sans feuilles caulinaires, que je distribue à cause de leur authenticité !

690-R. MOUGEOTII Billot ; R. casius $\times$ bifrons Sud. - Alsace (Müller).

Echantillons authentiques de $R$. roseiflorus Müll., provenant de plusieurs localités et dépourvus de feuilles caulinaires.

Müller avait reconnu lui-même que sa plante était identique à celle de Billot ( $\left.n^{\circ} 689\right)$.

691 - R. MOUGEOTII Billot; R. cesius $\times$ bifrons. - Silésie (Spribille).

Le $R$. Aschersonii Sprib., bien que rappelant un peu les hybrides de $R$.candicans, doit dériver du R.bifrons, dont l'intervention rend compte de ia coloration rose des fleurs et de la dentelure fine des feuilles.

692 - R. MOUGEOTIl Billot. ; R. cæesius $\times$ bifrons. - Poméranie (Holzfuss).

Forme moyenne, que j'ai reçue du collecteur sous le nom de $R$. ambifarius.

$\mathbf{6 9 3}$ - R. HEMISTENOPETALUS Sud.; $R$. stenopetalus $\times$ casius. - France : Valois (Questier). 


\section{$-145-$}

69\& - R. EXOTERICUS Sud. ; R. lacertosus X casius. - France : Tarn (H. Sudre).

Avec le $R$. lacertosus. Stérile.

695 - R. VIRGULTORUM P.-J. Müll. ; R. candicans $\times$ casius. - Alsace (Müller).

C'est là la forme appelée $R$. fasciculatus par Müller (p. p.) et rappelant le $R$. candicans var. coarctatus. Quelques parts sont dépourvues de feuilles caulinaires. - Müller avait récolté cette plante dans son jardin, à Wissembourg.

696-'R. AMBIFARIUS Müll. ; $R$. thyrsanthus $\times$ cresius. - Saxe (H. Hofmann).

69 8 - R. LEUCOPHÆUS P.-J. Müll.! R. casius $\times$ tomentosus Sud. - Alsace (Müller).

698. - R. LEUCOPHEUS P.-J. Müll. I; R. cœesius $\times$ tomentosus Sud. - France: Hte Garonne (H. Sudre).

Feuilles de l'extrémité des turions tomentelleuses en dessus.

699 - R. DELTOIDEUS P.-J. Müll.; $R$. tomentosus Lloydianus $\times$ ccesius. - Alsace (Müller).

รоO - R. PSEUDODEGENER Sud. ; $R$. coesius $Х$ tomentosus Lloydianus. - France : Tarn (H. Sudre).

\section{H. SUDRE.}

Toulouse, juillet 1916 .

Albi.-Impr. A. Nouguies, rue de l'Hôtel-de-Ville. 33 




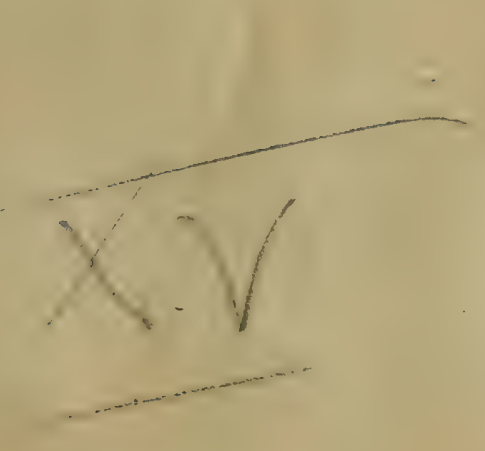

$$
\text { - }
$$





\title{
BATOTHECA EUROP瓜A
}

PUBLIÉ PAR

\author{
H. SUDRE \\ Professeur à l'Lcole Normale de Toulouse (Irance) \\ 3 , rue André Délieux
}

FASCIGULE XV - 1917

Parait par fascicules annuels de 50 numéros. Les collabo. rateurs qui fournissent 5 numéros convenus d'avance et préparés à 25 parts reçoivent un fascicule en échange; ils payent une cotisation annuelle de $2 \mathrm{fr}$. pour couvrir les frais d'impression des étiquettes et le port du fascicule qu'ils reçoivent. Chaque exemplaire doit comprenire, autant que possible, deux rameaux, l'un florifère et l'autre fructifère, plusieurs feuilles caulinaires et quelques pétales préparés à part. Les espèces déjà distribuées ne sont admises que si elles diffèrent quelque peu des formes publiées.

\section{Prix du fascicule : 25 fr.}

\section{COLLECTEURS POUR LE $15^{\circ}$ FASGICULE}

C. Bicknell et Pollini à Bordighera (Italie), no 733.

Dr Biau, à Castres (Tarn), nos 715, 738.

D Bouly de Lesdain, à Dunkerque (Nord), no 710.

G. Bouvet, à Angers' (Maine-et-Loire), nos'715, 738 .

J.-B. Charbonnel, La Chapelie-Laurent (Cantal). nos 740, 744, 745.

Dr Chassagne, à Lezoux (Puy-de-Dòme), nos 746-8.

$\times$ Ohaboisseau (Vienne), no 716 .

$\times$ F. Graver (Belgique, $\mathrm{r}^{\circ} 724$.

A. Henry, Le Mans (Sarthe), nos 708, 711.

H. Hofmann, à Grossenhail (Saxe), nos 714, 719, 720, 729, 730, 734, 736, 737, 739 .

z. Kaufmann, Nürnberg (Bavière), no 750.

Dr Kinscher, Steinach (Thuringe), n $717-8$.

F. Kretzer, Braunschweig, n $703,706$.

$\times$ s. Kupcok, Bakabanya (Hongrie), no 742 .

$\times$ P.-J. Müller (Alsace), nos 702, 731 .

$\times$ Quostier (Valois), nos 705, 709, 725 .

Dr H. Sabransky (Sty rie), nos 727, 732, 741, 749.

Spribille, Breslau, (Silésie), nos 704, 713, 721-3, 726, 728 .

H. Sudre, à Toulouse, nos 701, 735, 743 .

\section{OBSERVATIONS}

Les collaborateurs sont priés de ne pas récolter les espèces déjà publiées dans le Batothesa. Ce fascıcule renferme 28 Rubus'non distribués dans des exsiccatu numérotés.

H. SUDRE. 
Sect. V. - Triviales P.-J. Müll.

No 11 - R. ALBIGENSIS Sud.; R. (cosims $X$ Lloydianus $) \times$ ulmifolius. - France : Tarn (H. Sudre).

No 1 - R. Scabrosus P.-J. Müll.!; R. vestitus $X$ coesius Sud. - Alsace (Müller).

La pilosité des turions et surtout de la face supérieure des feuilles montre l'influence du $R$. vestitus.

No $7 \mathbf{O B}-\mathrm{R}$. IMITABILIS K. Frid. $-\boldsymbol{R}$. mucronifer $\times$ coesius Sud. - Allemagne: S. Harz (F. Kretzer).

La moitié des spécimens récoltés par M. Kretzer appartenaient au $R$. Balfourianus Blox., d'où des parts un peu maigres.

No 104 - R. IMITABILIS K. Frid.; $R$. mucronifer $\times$ coesius Sud. - Allemagne : Hannover (Spribille).

No 705 - R ASPRETICOLUS L. et M. !; $R$. Mueller $\imath \times$ coesius Sud. - France : Valois (Questier).

Noro6 - R. CUSPIDATUS Müll.; $R$. Radula X casius Sud. - S. Harz (F. Kretzer).

M. F. Kretzer m'a adressé cette plante sous le nom de $R$. rudis $\times$ vestitus, mais elle appartient manifestement aux $R$. triviales.

No $70 \boldsymbol{\gamma}-\mathrm{R}$. PLATYANTHUS Sud.; $\boldsymbol{R}$. uncinatus $\times$ coesius.- France: Vosges (Biau).

Au milieu des parents ( $D^{r}$ Biau).

No $7 \mathbf{0} 8$ - R. DISSOLUTUS Sud. ; R. ericetorumX coesius Sud. - France : Loire inf. (Henry).

No $\boldsymbol{\gamma O 9}-\mathrm{R}$. HORRIFACTUS M. et L. ; $R$. papulosus $\times$ casius Sud. - France : Oise (Questier).

La plupart des spécimens étaient mélangés au $R$. papulosus. 
No $1-$ R. HIRTICAULIS L. et M.; $R$. Genevieri $\times$ ccesius Sud. - France: Seine-et-Oise (B. de Lesdain).

No $11-$ R. DISCERPTIFORMIS Sud. ; $R$. discerptus $\times$ casius. - France : Sarthe (A. Henry).

$\mathrm{N}^{\circ} \geq-$ R. SEMIMICANS Sud.; $R$. micans $\mathrm{X}$ casius. - France : Vosges (Dr Biau).

Il est possible que ce soit la var virenticanus qui ait produit cet hybride.

$\mathrm{N}^{\circ} \boldsymbol{\gamma} \mathbf{3}-\mathrm{R}$. FABRIMONTANUS Spribille; $\boldsymbol{R}$. obscurus erraticus Xcesius. - Silésie (Spribille).

No 1 - R. OMALOIDES Sud. ; $R$. omalus X cesius. - Saxe (Hofmann).

Je n'ai pas vu de Saxe le $R$. omalus, mais comme il est fréquent en Bavière et en Silésie, il est probable qu'i existe aussi en Saxe.

No $15-R$. DECORATUS Sud. et Bour.; $R$. melanoxylon Xcesius. - France: Anjou (Bouvet).

No $16-\mathrm{R}$. ADENOLEUCUS Chab. ! R. rudis X coesius. - France : Vienne (Chaboisseau).

Spécimens authentiques.

No 1 r - R. OLEOGETON Focke; $R$. KuhleriX coesius. - Silésie (Kinscher).

No $18-\mathrm{R}$. OREOGETON Focke ; $R$. KohleriX casius. - Silésie (Kinscher).

C'est le $\boldsymbol{R}$. laticors Kinsch. L'aculéation de l'inflorescence est celle du $R$. Koehleri Wh.

No $19-$ R. OREOGETON Focke ; $R$. KoehleriX cœsius. - Saxe (Hofmann).

Dentelure plus fine que dans les $n^{\circ 8} 717,718$, mais feuilles caul. 5-nées. Ronce vigoureuse, me paraissant dériver aussi du $R$. Koehleri ou de quelque forme de ce groupe. 
No $\boldsymbol{\gamma} \boldsymbol{2 0}-\mathrm{R}$. IDAIFORMIS Sud. et Hofm. $R$. oreogeton $\times$ Idaus. - Saxe (H. Hofmann).

L'influence du $R$. Idoeus n'est pas douteuse ; l'aculéation est celle du n० 719 ; toutefois les glandes manquent. Forme très curieuse !

No $\boldsymbol{\tau} 2 \mathbf{1}-\mathrm{R}$. PILOSUS Wh. ; R. scaber $\times$ coesius Sud. - Silésie (Spribille).

Les spécimens de Weihe, qui sont du reste peu homogènes, ont le turion un peu pubescent. Je crois qu'ils ont la même origine que ceux-ci, que M. Spribille appelle $R$. polycarpiformis ( $R$. polycarpus Fl. Schles.)

No $\boldsymbol{\gamma} 2 \boldsymbol{2}-\mathrm{R}$. ORTHACANTHUS Wimmer; $\boldsymbol{R}$. Schleicheri $\times$ coesius Sud. - Silésie (Spribille).

N. $\mathbf{2} 3$ - R. CAPITULATUS Utsch; $R$. Bellardii Xcoesius Sud. - Silésie (Spribille).

Forme des feuilles caul. rappelant le $R$. Bellardii !

No 2 - R. SEMIRIVULARIS Sud. ; $k$. rivularis $\times$ casius. - Belgique (Gravet).

No $\boldsymbol{7} \mathbf{2}-\mathrm{R}$. SPINETORUM M. L. $! ; R$. serpens angustifrons $\times$ casius Sud. - France: Valois (Questier).

No $\boldsymbol{7} \mathbf{2 6}$ - R. SEEBERGENSIS Pfuhl ; $R$. leptadenes $\times$ cœesius Sud. - Silésie (Spribille).

Forme grèle, rappelant beaucoup le $R$. serpens (leptadenes).

No $\boldsymbol{\gamma} \boldsymbol{\gamma}$ - R. HOLOSERICEUS Vest. ; R. Villarsianus Xtomentosus Sud. - Styrie (Sabransky).

Tient a la fois des $R$. hirtus, cosius et tomentosus.

No $2 \mathbf{2 8}-$ R. SUBTILISSIMUS Sud.; $R$. cosius X hirtus $\mathrm{f}^{\mathrm{a}}$. - Silésie (Spribille).

Ce $\mathrm{n}^{0}$ pourrait être un $R$. ccesius $\times$ hirtus tenuidentatus. 
Idæobatus Focke

No 29 - R. IDAEUS L. f. phyllanthus Lge. - Saxe (Hofmann).

No 30 - R. IDAEUS L. var. denudatus Sch. et Sp. - Saxe (Hofmann).

No $31-\mathrm{R}$. ID EOIDES Ruthe; R. Idoeus $\times$ coesius F. - Alsace (Müller).

Spécimens authentiques de $R$. coesio-Idous P.-J. Müll. !

No $32-R$. IDEOIDES Ruthe; $R$. Idœus $\times$ casims F. - Styrie (Sabransky).

\section{Cylactis Raf.}

No 333 - F. AAXATLIS L. - Italie (Bicknell et Pollini).

Chamæmorus F.

No 3 A - R. CHAM AEMORUS L. Riesengebirge (Hofmann).

Sect. Silvatici Müll.

No735 - R. quencetioolus Sud. - France : Hte-Garonne (H. Sudre).

No 366 - R. PXIR AMID CIO Kalt. var. sitiens Hofm. - Saxe (Hofmánn).

Nor 3 - R. PXIRAMIDACIS Kalt. var. sitiens Hofm. - Saxe (Hofmann).

Voisin de la var. similatus, mais dentelure plus fine, fleurs plus petites, feuilles vertes en dessous. 
No 738 - RE. ART ENTEUS W. et N. var. brevistamineus Sud. - France: Anjou (Bouvet).

D'après M. Bouvet ce n'est pas une forme accidentelle car il est répandu dans la région de St-Léger et de St-Jean de Linières.

No 399 - IR. HMI REICATUS Hort. v. saxonicus (Hofm.). - Saxe (Hofmann).

Sect. Discolores Müll.

No $\& \mathbf{R}-\mathrm{R}$. HOLOLEUCOIDES Sud.; $R$. propinquus $\times$ Lloydianus. - France : Cantal (Charbonnel).

Sect. Appendiculati Gen.

Vestiti Focke.

No $1-$ R. PASTORALIS Sabrs. ; Sud. Rub. Eur. p. 262 (1913). - Styrie (Sabransky).

Parait stérile; mais origine douteuse.

No 22 - Cafiochii $F$. var. mulleus (Kpk.). - Hongrie (Kupcok).

$\mathrm{N}_{0}$ 月3 - TR. MUECLARI Lef. var. cinerellus Sud. - France: Hte-Garonne (H. Sudre).

No - R. FLAVIDULUS Sud.; R. ColemanniiX Lloydianus. - France : Cantal (Charbonnel).

RAdUlæ Focke.

$\mathrm{N}^{\circ}$ g 5 - Re. oubrotund sud. var. ramosipes. - France : Cantal (Charbonnel).

No $\mathbf{A}-\mathrm{R}$. abruptifolius Sud. var. supervestitus. - France: Puy-de-Dôme (Chassagne).

No \& $/$ - R. CHASSAGNEI Sud. ; R. abruptifolius var. supervestitus Xalbiflorus. - France: Puyde-Dôme (Chassagne). 
J'avais d'abord supposé que la plante dérivait du $R$. caliginosus, mais elle croît pêle-mêle avec le $n^{\circ}$ 746 , dont elle provient vraisemblablement.

$\mathrm{N}^{\circ} \mathbf{8}-\mathrm{R}$. SEMISUPERVESTITUS Sud.; $R$. abruptifolius var. supervestilus $\times$ ulmifolius. - France : Puy-de-Dôme (Chassagne).

N 49 - TE. GRA AUTATUG M. et L. var. lucifugus (Sabrs.) - Styrie (Sabransky).

Glandulosi Müll.

No 550 - Re. insequabilis Sud. var. incquabiliformis. - Bavière (E. Kaufmann).

\section{H. SUDRE.}

Toulouse, avril $191 \%$.

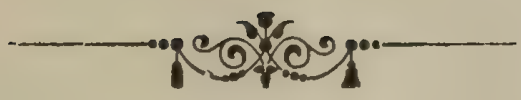










